\title{
Spatial Sobolev regularity for stochastic Burgers equations with additive trace class noise
}

\author{
Arnulf Jentzen ${ }^{1}$, Felix Lindner ${ }^{2}$, and Primož Pušnik ${ }^{3}$ \\ ${ }^{1}$ Seminar for Applied Mathematics, Department of Mathematics, \\ ETH Zurich, Switzerland, e-mail: arnulf.jentzen@sam.math.ethz.ch \\ ${ }^{2}$ Institute of Mathematics, Faculty of Mathematics and Natural Sciences, \\ University of Kassel, Germany, e-mail: lindner@mathematik.uni-kassel.de \\ 3 Seminar for Applied Mathematics, Department of Mathematics, \\ ETH Zurich, Switzerland, e-mail: primoz.pusnik@sam.math.ethz.ch
}

August 20, 2019

\begin{abstract}
In this article we investigate the spatial Sobolev regularity of mild solutions to stochastic Burgers equations with additive trace class noise. Our findings are based on a combination of suitable bootstrap-type arguments and a detailed analysis of the nonlinearity in the equation.
\end{abstract}

\section{Contents}

1 Introduction

1.1 General setting . . . . . . . . . . . . . . . . . . .

2 Pathwise solvability for a class of random ODEs

3 Strong a priori bounds based on bootstrap-type arguments

4 Properties of the nonlinearity

4.1 Auxiliary results on Sobolev and interpolation spaces . . . . . . . . . . . . 19

4.2 Analysis of the nonlinearity . . . . . . . . . . . . . . . 27

5 Existence and uniqueness of mild solutions to stochastic Burgers equations 36 


\section{Introduction}

In the literature, there are nowadays various results on existence, uniqueness, and regularity of solutions to stochastic Burgers equations. In particular, existence and uniqueness results for mild solutions to stochastic Burgers equations with additive space-time white noise and zero Dirichlet boundary conditions on the unit interval $(0,1)$ taking values in the space $L^{p}((0,1), \mathbb{R})$ for $p \in[2, \infty)$, in the space $\mathcal{C}([0,1], \mathbb{R})$ of continuous functions, and in $L^{2}((0,1), \mathbb{R})$-Sobolev-type spaces of order up to $1 / 2$ can be found, e.g., in Da Prato et al. [7], Blömker \& Jentzen [4], Jentzen et al. [23], and Mazzonetto \& Salimova [31. Results on existence, uniqueness, and regularity of solutions to stochastic Burgers equations with multiplicative space-time white noise and zero Dirichlet boundary conditions on the unit interval have been established, e.g., in Da Prato \& Gatarek [8] and Gyöngy [15]. Existence, uniqueness, and regularity results for solutions to stochastic Burgers equations on the whole real line can be found, e.g., in Bertini et al. [3], Gyöngy \& Nualart [16], Kim [25], and Lewis \& Nualart [28]. Results on existence, uniqueness, and regularity of mild solutions to stochastic Burgers equations driven by Lévy noise are presented, e.g., in Dong \& Xu [12] and Hausenblas \& Giri [17]. We also refer to Brzeźniak et al. [6], Da Prato \& Zabczyk [10, Section 14], Da Prato \& Zabczyk [11, Section 13.9], Röckner et al. [34], and the references mentioned therein for further existence, uniqueness, and regularity results for stochastic Burgers-type equations. In this paper, we present a higher order regularity result for stochastic Burgers equations with additive trace-class noise and zero Dirichlet boundary conditions on the unit interval $(0,1)$. More specifically, in Theorem 5.10, which is the main result of this article, we establish the unique existence of mild solutions taking values in $L^{2}((0,1), \mathbb{R})$-Sobolev-type spaces of order up to 2. A slightly simplified version of our main result is given in the following theorem.

Theorem 1.1. Let $\left(H,\langle\cdot, \cdot\rangle_{H},\|\cdot\|_{H}\right)$ be the $\mathbb{R}$-Hilbert space of equivalence classes of Lebesgue-Borel square-integrable functions from $(0,1)$ to $\mathbb{R}$, let $A: D(A) \subseteq H \rightarrow H$ be the Laplacian with zero Dirichlet boundary conditions on $H$, let $\left(H_{r},\langle\cdot, \cdot\rangle_{H_{r}},\|\cdot\|_{H_{r}}\right), r \in \mathbb{R}$, be a family of interpolation spaces associated to $-A$, let $\beta \in(-1 / 4, \infty), \gamma \in(1 / 4, \min \{1,1 / 2+\beta\}), T \in(0, \infty), \xi \in H_{1}$, $B \in \operatorname{HS}\left(H, H_{\beta}\right)$, let $(\Omega, \mathcal{F}, \mathbb{P})$ be a probability space, and let $\left(W_{t}\right)_{t \in[0, T]}$ be an $\operatorname{Id}_{H}$-cylindrical Wiener process. Then

(i) there exists a unique continuous function $F: H_{1 / 8} \rightarrow H_{-1 / 2}$ which satisfies for every $v \in H_{1 / 2}$ that $F(v)=-v^{\prime} v$ and

(ii) there exists an up to indistinguishability unique stochastic process $X:[0, T] \times \Omega \rightarrow H_{\gamma}$ with continuous sample paths which satisfies that for every $t \in[0, T]$ it holds $\mathbb{P}$-a.s. that

$$
X_{t}=e^{t A} \xi+\int_{0}^{t} e^{(t-s) A} F\left(X_{s}\right) d s+\int_{0}^{t} e^{(t-s) A} B d W_{s}
$$

Theorem 1.1 is a direct consequence of Theorem [5.10 (with $T=T, \varepsilon=1-\gamma, c_{0}=1$, $c_{1}=-1, \beta=\beta, \gamma=\gamma, A=A, H_{r}=H_{r},(\Omega, \mathcal{F}, \mathbb{P})=(\Omega, \mathcal{F}, \mathbb{P}),\left(W_{t}\right)_{t \in[0, T]}=\left(W_{t}\right)_{t \in[0, T]}, B=B$, $\xi=\left(\Omega \ni \omega \mapsto \xi \in H_{1}\right)$ for $r \in \mathbb{R}, \gamma \in(1 / 4, \min \{1,1 / 2+\beta\})$ in the notation of Theorem 5.10]) in Section 5 below. Note that the assumption in Theorem 1.1 above that $\left(H_{r},\langle\cdot, \cdot\rangle_{H_{r}},\|\cdot\|_{H_{r}}\right)$, $r \in \mathbb{R}$, is a family of interpolation spaces associated to $-A$ ensures that for every $r \in[0, \infty)$ it holds that $\left(H_{r},\langle\cdot, \cdot\rangle_{H_{r}},\|\cdot\|_{H_{r}}\right)=\left(D\left((-A)^{r}\right),\left\langle(-A)^{r}(\cdot),(-A)^{r}(\cdot)\right\rangle_{H},\left\|(-A)^{r}(\cdot)\right\|_{H}\right)$. The equation in (11) above is referred to as stochastic evolution equation (SEE) or stochastic partial differential equation (SPDE) in the scientific literature and, roughly speaking, there are mainly three 
common approaches for describing and analyzing solutions of SPDEs: $(i)$ the martingale measure approach (cf., e.g., Walsh [39]), (ii) the variational (weak solution) approach (cf., e.g., Grecksch \& Tudor [14], Liu \& Röckner [29], Prévôt \& Röckner [33], and Rozovskiü [35]), and (iii) the semigroup (mild solution) approach (cf., e.g., Da Prato \& Zabczyk [10, 11], Grecksch \& Tudor [14, and Liu \& Röckner [29]) in the literature. Theorem [1.1] and most of the other results in this article are formulated within the semigroup approach. The proof of Theorem 1.1 and Theorem 5.10, respectively, is mainly based on combining Corollary 2.4, Lemma 4.16, Corollary 4.18, Lemma 5.3, and Lemma 5.8, Corollary 2.4 establishes the unique existence of suitable spatial spectral Galerkin approximations of stochastic Burgers equations (see the proof of Lemma 5.9 and (263) in the proof of Theorem 5.10 below). An existence and uniqueness result for stochastic differential equations (SDEs) similar to Corollary 2.4 can be found, e.g., in Liu \& Röckner 29, Theorem 3.1.1]. Lemma 4.16 and Corollary 4.18 (cf., e.g., Blömker \& Jentzen [4, Lemma 4.7]) prove that the involved nonlinearity $F$ (see item (ii) in Theorem 1.1 above) satisfies specific local Lipschitz conditions (see (273) in the proof of Theorem 5.10 below). Lemma 5.3 establishes appropriate pathwise uniform a priori bounds for the spatial spectral Galerkin approximations of the considered stochastic Burgers equation (see (280) in the proof of Theorem 5.10 below). Its proof is based on consecutive applications of suitable bootstrap-type arguments in Section 3 to establish appropriate a priori bounds for the solution processes of the considered SDEs in higher order smoothness spaces. Related bootstrap-type arguments can be found, e.g., in Jentzen \& Pušnik [21, Section 3], Jentzen \& Röckner [22, Theorem 1], and Zhang [40, Section 3]. Lemma 5.8 (cf., e.g., Blömker \& Jentzen [4, Lemma 4.3]) demonstrates pathwise uniform convergence rates of spatial spectral Galerkin approximations of the considered stochastic integral (see (277) in the proof of Theorem 5.10 below). Its proof is essentially based on an application of the factorization method for stochastic convolutions in Lemma 5.6. Combining these mentioned results with the existence and uniqueness result in Blömker \& Jentzen [4, Theorem 3.1] proves Theorem 5.10.

The remainder of this article is structured as follows. In Section 2 we recall some elementary existence and uniqueness results for random ordinary differential equations (ODEs). In Section 3 we employ bootstrap-type arguments to establish suitable a priori bounds for certain approximation processes. In Subsection 4.1 we recall some elementary properties of Sobolev-Slobodeckij and interpolation spaces. In Subsection 4.2 we recall and derive several auxiliary results on the regularity properties of the nonlinearity appearing in the stochastic Burgers equation. In Section 5 we combine the results in Sections 24 to establish the main result of this article in Theorem 5.10 below.

\subsection{General setting}

Throughout this article the following setting is frequently used.

Setting 1.2. For every measurable space $\left(\Omega_{1}, \mathcal{F}_{1}\right)$ and every measurable space $\left(\Omega_{2}, \mathcal{F}_{2}\right)$ let $\mathcal{M}\left(\mathcal{F}_{1}, \mathcal{F}_{2}\right)$ be the set of all $\mathcal{F}_{1} / \mathcal{F}_{2}$-measurable functions from $\Omega_{1}$ to $\Omega_{2}$, let $\left(H,\langle\cdot, \cdot\rangle_{H},\|\cdot\|_{H}\right)$ be a separable $\mathbb{R}$-Hilbert space, let $\mathbb{H} \subseteq H$ be a non-empty orthonormal basis of $H$, let $\mathfrak{v}: \mathbb{H} \rightarrow \mathbb{R}$ be a function which satisfies $\sup _{h \in \mathbb{H}} \mathfrak{v}_{h}<0$, let $A: D(A) \subseteq H \rightarrow H$ be the linear operator which satisfies $D(A)=\left\{v \in H: \sum_{h \in \mathbb{H}}\left|\mathfrak{v}_{h}\langle h, v\rangle_{H}\right|^{2}<\infty\right\}$ and $\forall v \in D(A): A v=\sum_{h \in \mathbb{H}} \mathfrak{v}_{h}\langle h, v\rangle_{H} h$, and let $\left(H_{r},\langle\cdot, \cdot\rangle_{H_{r}},\|\cdot\|_{H_{r}}\right), r \in \mathbb{R}$, be a family of interpolation spaces associated to $-A$ (cf., e.g., [37, Section 3.7]).

Note that the assumption in Setting 1.2 above that $\left(H_{r},\langle\cdot, \cdot\rangle_{H_{r}},\|\cdot\|_{H_{r}}\right), r \in \mathbb{R}$, is a family of interpolation spaces associated to $-A$ ensures that for every $r \in[0, \infty)$ it holds that 
$\left(H_{r},\langle\cdot, \cdot\rangle_{H_{r}},\|\cdot\|_{H_{r}}\right)=\left(D\left((-A)^{r}\right),\left\langle(-A)^{r}(\cdot),(-A)^{r}(\cdot)\right\rangle_{H},\left\|(-A)^{r}(\cdot)\right\|_{H}\right)$.

\section{Pathwise solvability for a class of random ODEs}

In this section we analyze in Corollary 2.4 the solvability of a specific class of abstract random ODEs. The considered equations can be thought of as spectral Galerkin discretizations in space of an underlying stochastic Burgers equation. Corollary 2.4 is based on an elementary and essentially well-known pathwise existence and uniqueness result for random ODEs with non-globally Lipschitz continuous coefficient functions presented in Lemma 2.3 (cf., e.g., Liu \& Röckner [29, Theorem 3.1.1]). In addition, we also recall elementary results on measurability in Lemma 2.1 (see, e.g., Aliprantis \& Border [1, Lemma 4.51]) and Lemma 2.2 (cf., e.g., in Klenke [26, Theorem 14.16]). For the sake of completeness we include the proof of Lemma 2.2 .

Lemma 2.1. Let $(\Omega, \mathcal{F})$ be a measurable space, let $\left(X, d_{X}\right)$ be a separable metric space, let $\left(Y, d_{Y}\right)$ be a metric space, let $f: X \times \Omega \rightarrow Y$ be a function, assume for every $x \in X$ that $\Omega \ni \omega \mapsto f(x, \omega) \in$ $Y$ is $\mathcal{F} / \mathcal{B}(Y)$-measurable, and assume for every $\omega \in \Omega$ that $(X \ni x \mapsto f(x, \omega) \in Y) \in \mathcal{C}(X, Y)$. Then it holds that $f: X \times \Omega \rightarrow Y$ is $(\mathcal{B}(X) \otimes \mathcal{F}) / \mathcal{B}(Y)$-measurable.

Note that for every topological space $(X, \tau)$ it holds that $\mathcal{B}(X)$ is the smallest sigma-algebra on $X$ which contains all elements of $\tau$.

Lemma 2.2. Let $\left(X,\|\cdot\|_{X}\right)$ be an $\mathbb{R}$-Banach space, let $(\Omega, \mathcal{F})$ be a measurable space, let $a \in \mathbb{R}$, $b \in(a, \infty)$, let $f:[a, b] \times \Omega \rightarrow X$ be a strongly $(\mathcal{B}([a, b]) \otimes \mathcal{F}) /\left(X,\|\cdot\|_{X}\right)$-measurable function, assume for every $\omega \in \Omega$ that $\int_{a}^{b}\|f(s, \omega)\|_{X} d s<\infty$, and let $F: \Omega \rightarrow X$ be the function which satisfies for every $\omega \in \Omega$ that $F(\omega)=\int_{a}^{b} f(s, \omega) d s$. Then it holds that $F$ is strongly $\mathcal{F} /\left(X,\|\cdot\|_{X}\right)$ measurable.

Proof of Lemma [2.2. Throughout this proof let $\lambda: \mathcal{B}(\mathbb{R}) \rightarrow[0, \infty]$ be the Lebesgue-Borel measure on $\mathbb{R}$, let $\mathcal{C} \subseteq(\mathcal{B}([a, b]) \otimes \mathcal{F})$ be the set given by

$$
\mathcal{C}=\left\{C \in(\mathcal{B}([a, b]) \otimes \mathcal{F}):\left(\Omega \ni \omega \mapsto \int_{a}^{b} \mathbb{1}_{C}(s, \omega) d s \in \mathbb{R}\right) \text { is } \mathcal{F} / \mathcal{B}(\mathbb{R}) \text {-measurable }\right\}
$$

for every set $S$ let $\mathcal{P}(S)$ be the power set of $S$, for every set $S$ and every $\mathcal{A} \subseteq \mathcal{P}(S)$ let $\sigma_{S}(\mathcal{A})$ be the smallest sigma-algebra on $S$ which contains $\mathcal{A}$, and for every set $S$ and every $\mathcal{A} \subseteq \mathcal{P}(S)$ let $\delta_{S}(\mathcal{A})$ be the smallest Dynkin system on $S$ which contains $\mathcal{A}$. First, we intend to prove that

$$
\mathcal{C}=\mathcal{B}([a, b]) \otimes \mathcal{F}
$$

For this note that for every $A \in \mathcal{B}([a, b]), B \in \mathcal{F}, \omega \in \Omega$ it holds that

$$
\int_{a}^{b} \mathbb{1}_{A \times B}(s, \omega) d s=\int_{a}^{b} \mathbb{1}_{A}(s) \mathbb{1}_{B}(\omega) d s=\lambda(A) \mathbb{1}_{B}(\omega) .
$$

This ensures that

$$
\{A \times B: A \in \mathcal{B}([a, b]), B \in \mathcal{F}\} \subseteq \mathcal{C} \quad \text { and } \quad([a, b] \times \Omega) \in \mathcal{C} .
$$


The fact that $\{A \times B: A \in \mathcal{B}([a, b]), B \in \mathcal{F}\}$ is $\cap$-stable and Dynkin's Lemma therefore prove that

$$
\begin{aligned}
\mathcal{B}([a, b]) \otimes \mathcal{F} & =\sigma_{[a, b] \times \Omega}(\{A \times B: A \in \mathcal{B}([a, b]), B \in \mathcal{F}\}) \\
& =\delta_{[a, b] \times \Omega}(\{A \times B: A \in \mathcal{B}([a, b]), B \in \mathcal{F}\}) \\
& \subseteq \delta_{[a, b] \times \Omega}(\mathcal{C}) \subseteq \delta_{[a, b] \times \Omega}(\mathcal{B}([a, b]) \otimes \mathcal{F})=\mathcal{B}([a, b]) \otimes \mathcal{F}
\end{aligned}
$$

This shows that

$$
\delta_{[a, b] \times \Omega}(\mathcal{C})=\mathcal{B}([a, b]) \otimes \mathcal{F} .
$$

Moreover, note that for every $C \in \mathcal{C}, \omega \in \Omega$ it holds that

$$
\begin{aligned}
\int_{a}^{b} \mathbb{1}_{([a, b] \times \Omega) \backslash C}(s, \omega) d s & =\int_{a}^{b}\left(\mathbb{1}_{[a, b] \times \Omega}(s, \omega)-\mathbb{1}_{C}(s, \omega)\right) d s \\
& =\int_{a}^{b} \mathbb{1}_{[a, b] \times \Omega}(s, \omega) d s-\int_{a}^{b} \mathbb{1}_{C}(s, \omega) d s .
\end{aligned}
$$

This and (5) imply that for every $C \in \mathcal{C}$ it holds that

$$
(([a, b] \times \Omega) \backslash C) \in \mathcal{C} .
$$

Furthermore, note that the monotone convergence theorem proves that for all pairwise disjoint sets $C_{n} \in \mathcal{C}, n \in \mathbb{N}$, it holds that

$$
\begin{aligned}
\int_{a}^{b} \mathbb{1}_{\cup_{n \in \mathbb{N}} C_{n}}(s, \omega) d s & =\int_{a}^{b} \sum_{n=1}^{\infty} \mathbb{1}_{C_{n}}(s, \omega) d s \\
& =\int_{a}^{b} \lim _{k \rightarrow \infty} \sum_{n=1}^{k} \mathbb{1}_{C_{n}}(s, \omega) d s=\lim _{k \rightarrow \infty} \int_{a}^{b} \sum_{n=1}^{k} \mathbb{1}_{C_{n}}(s, \omega) d s .
\end{aligned}
$$

Therefore, we obtain that for all pairwise disjoint sets $C_{n} \in \mathcal{C}, n \in \mathbb{N}$, it holds that $\cup_{n \in \mathbb{N}} C_{n} \in \mathcal{C}$. Combining this, (5), and (9) implies that $\mathcal{C}$ is a Dynkin system on $[a, b] \times \Omega$. Combining this and (7) establishes (31). Next we intend to establish the statement of Lemma 2.2. For this observe that the fact that $f:[a, b] \times \Omega \rightarrow X$ is strongly $(\mathcal{B}([a, b]) \otimes \mathcal{F}) /\left(X,\|\cdot\|_{X}\right)$-measurable and, e.g., Prévôt \& Röckner [33, Lemma A.1.4] imply that there exist $(\mathcal{B}([a, b]) \otimes \mathcal{F}) / \mathcal{B}(X)$-measurable functions $f_{n}:[a, b] \times \Omega \rightarrow X, n \in \mathbb{N}$, which satisfy that

(a) it holds for every $n \in \mathbb{N}$ that $f_{n}([a, b] \times \Omega)$ is a finite set and

(b) it holds for every $\omega \in \Omega$ that

$$
\limsup _{n \rightarrow \infty}\left\|\int_{a}^{b} f_{n}(\omega, s) d s-\int_{a}^{b} f(\omega, s) d s\right\|_{X} \leq \limsup _{n \rightarrow \infty} \int_{a}^{b}\left\|f_{n}(\omega, s)-f(\omega, s)\right\|_{X} d s=0 .
$$

Note that item (国) shows that for every $n \in \mathbb{N}, s \in[a, b], \omega \in \Omega$ it holds that

$$
f_{n}(s, \omega)=\sum_{x \in f_{n}([a, b] \times \Omega)} x \mathbb{1}_{\left(f_{n}\right)^{-1}(\{x\})}(s, \omega) .
$$

The fact that for every $n \in \mathbb{N}$ it holds that $f_{n}([a, b] \times \Omega)$ is a finite set, the fact that for every $n \in \mathbb{N}$, $x \in X$ it holds that $\left(f_{n}\right)^{-1}(\{x\}) \in(\mathcal{B}([a, b]) \otimes \mathcal{F})$, and (33) hence prove that for every $n \in \mathbb{N}$ it holds that $\Omega \ni \omega \mapsto \int_{a}^{b} f_{n}(s, \omega) d s \in X$ is strongly $\mathcal{F} /\left(X,\|\cdot\|_{X}\right)$-measurable. Combining item (国), item (b) , and, e.g., Prévôt \& Röckner [33, item (i) of Proposition A.1.3] therefore establishes that $F$ is strongly $\mathcal{F} /\left(X,\|\cdot\|_{X}\right)$-measurable. The proof of Lemma 2.2 is thus completed. 
Lemma 2.3. Let $\left(H,\|\cdot\|_{H},\langle\cdot, \cdot\rangle_{H}\right)$ be a separable $\mathbb{R}$-Hilbert space, let $T \in(0, \infty), s \in[0, T)$, let $\left(\Omega, \mathcal{F}, \mathbb{P},\left(\mathbb{F}_{t}\right)_{t \in[s, T]}\right)$ be a filtered probability space, let $\xi: \Omega \rightarrow H$ be an $\mathbb{F}_{s} / \mathcal{B}(H)$-measurable function, let $f:[s, T] \times H \times \Omega \rightarrow H$ and $K:[s, T] \times(0, \infty) \times \Omega \rightarrow[0, \infty)$ be functions, assume for every $t \in[s, T], x \in H$ that $\Omega \ni \omega \mapsto f(t, x, \omega) \in H$ is $\mathbb{F}_{t} / \mathcal{B}(H)$-measurable, assume for every $\omega \in \Omega, r \in(0, \infty)$ that $([s, T] \times H \ni(t, x) \mapsto f(t, x, \omega) \in H) \in \mathcal{C}([s, T] \times H, H),([s, T] \ni$ $\left.t \mapsto K_{t}(r, \omega) \in[0, \infty)\right) \in \mathcal{C}([s, T],[0, \infty))$, and $\sup _{t \in[s, T]} \sup _{x \in H,\|x\|_{H} \leq r}\|f(t, x, \omega)\|_{H}<\infty$, and assume for every $t \in[s, T], x, y \in H, \omega \in \Omega, r \in(0, \infty)$ with $\max \left\{\|x\|_{H},\|y\|_{H}\right\} \leq r$ that $2\langle x, f(t, x, \omega)\rangle_{H} \leq K_{t}(1, \omega)\left(1+\|x\|_{H}^{2}\right)$ and

$$
2\langle x-y, f(t, x, \omega)-f(t, y, \omega)\rangle_{H} \leq K_{t}(r, \omega)\|x-y\|_{H}^{2} .
$$

Then

(i) there exists a unique function $X:[s, T] \times \Omega \rightarrow H$ which satisfies for every $t \in[s, T], \omega \in \Omega$ that $\left([s, T] \ni u \mapsto X_{u}(\omega) \in H\right) \in \mathcal{C}([s, T], H)$ and

$$
X_{t}(\omega)=\xi(\omega)+\int_{s}^{t} f\left(u, X_{u}(\omega), \omega\right) d u
$$

and

(ii) it holds that $X:[s, T] \times \Omega \rightarrow H$ is $\left(\mathbb{F}_{t}\right)_{t \in[s, T]}$-adapted.

Proof of Lemma [2.3. Throughout this proof let $X^{n}:[s, T] \times \Omega \rightarrow H, n \in \mathbb{N}$, be the functions which satisfy for every $n \in \mathbb{N}, k \in\{0,1, \ldots, n-1\}, t \in\left(s+\frac{k(T-s)}{n}, s+\frac{(k+1)(T-s)}{n}\right], \omega \in \Omega$ that $X_{s}^{n}(\omega)=\xi(\omega)$ and

$$
X_{t}^{n}(\omega)=X_{s+(k(T-s) / n)}^{n}(\omega)+\int_{s+(k(T-s) / n)}^{t} f\left(u, X_{s+(k(T-s) / n)}^{n}(\omega), \omega\right) d u
$$

let $L:(0, \infty) \times \Omega \rightarrow[0, \infty)$ be the function which satisfies for every $r \in(0, \infty), \omega \in \Omega$ that

$$
L_{r}(\omega)=\sup _{t \in[s, T]} \sup _{h \in H,\|h\|_{H} \leq r}\|f(t, h, \omega)\|_{H},
$$

let $\kappa: \mathbb{N} \times[s, T] \rightarrow[s, T]$ be the function which satisfies for every $n \in \mathbb{N}, k \in\{0,1, \ldots, n-1\}$, $t \in\left(s+\frac{k(T-s)}{n}, s+\frac{(k+1)(T-s)}{n}\right]$ that $\kappa(n, s)=s$ and

$$
\kappa(n, t)=s+\frac{k(T-s)}{n},
$$

let $\mathcal{K}:[s, T] \times(0, \infty) \times \Omega \rightarrow[0, \infty)$ and $\alpha:[s, T] \times(0, \infty) \times \Omega \rightarrow[0, \infty)$ be the functions which satisfy for every $t \in[s, T], r \in(0, \infty), \omega \in \Omega$ that

$$
\mathcal{K}_{t}(r, \omega)=\max \left\{K_{t}(r, \omega), L_{r}(\omega)\right\} \quad \text { and } \quad \alpha_{t}(r, \omega)=\int_{s}^{t} \mathcal{K}_{u}(r, \omega) d u
$$

let $\tau^{n}:(0, \infty) \times \Omega \rightarrow[0, T], n \in \mathbb{N}$, be the functions which satisfy for every $n \in \mathbb{N}, r \in(0, \infty)$, $\omega \in \Omega$ that

$$
\tau_{r}^{n}(\omega)=\inf \left(\{T\} \cup\left\{t \in[0, T]:\left\|X_{t}^{n}(\omega)\right\|_{H} \geq r\right\}\right),
$$

and let $p^{n}:[s, T] \times \Omega \rightarrow H, n \in \mathbb{N}$, be the functions which satisfy for every $n \in \mathbb{N}, t \in[s, T]$, $\omega \in \Omega$ that

$$
p_{t}^{n}(\omega)=X_{\kappa(n, t)}^{n}(\omega)-X_{t}^{n}(\omega) .
$$


First, we establish item (ii). For this note that for every $r \in(0, \infty), n \in \mathbb{N}, \omega \in \Omega, t \in\left[s, \tau_{r}^{n}(\omega)\right]$ it holds that

$$
\begin{aligned}
& \left\|p_{t}^{n}(\omega)\right\|_{H} \leq \int_{\kappa(n, t)}^{t}\left\|f\left(u, X_{\kappa(n, u)}^{n}(\omega), \omega\right)\right\|_{H} d u \\
& \leq \int_{\kappa(n, t)}^{t} L_{r}(\omega) d u \leq(t-\kappa(n, t)) L_{r}(\omega) \leq \frac{(T-s)}{n} L_{r}(\omega)<\infty .
\end{aligned}
$$

This ensures for every $r \in(0, \infty), \omega \in \Omega$ that

$$
\lim \sup _{n \rightarrow \infty} \sup _{t \in[s, T]} \mathbb{1}_{\left[s, \tau_{r}^{n}(\omega)\right]}(t)\left\|p_{t}^{n}(\omega)\right\|_{H}=0 .
$$

The dominated convergence theorem hence shows that for every $r \in(0, \infty), \omega \in \Omega$ it holds that

$$
\lim \sup _{n \rightarrow \infty} \int_{s}^{T} \mathbb{1}_{\left[s, \tau_{r}^{n}(\omega)\right]}(u)\left\|p_{u}^{n}(\omega)\right\|_{H} \mathcal{K}_{u}(r, \omega) d u=0
$$

In the next step we observe that for every $t \in[s, T], n \in \mathbb{N}, \omega \in \Omega$ it holds that

$$
X_{t}^{n}(\omega)=\xi(\omega)+\int_{s}^{t} f\left(u, X_{\kappa(n, u)}^{n}(\omega), \omega\right) d u .
$$

Furthermore, note that the fact that for every $\omega \in \Omega, x \in H$ it holds that $([s, T] \ni u \mapsto$ $f(u, x, \omega) \in H) \in \mathcal{C}([s, T], H)$ and, e.g., [19, Corollary 2.7] (with $V=H, W=\mathbb{R}, a=s, b=T$, $\phi=\left([s, T] \times H \ni(t, x) \mapsto\|x\|_{H}^{2} e^{-\alpha_{t}(1, \omega)} \in \mathbb{R}\right), f=\left([s, T] \ni t \mapsto f\left(t, X_{\kappa(n, t)}^{n}(\omega), \omega\right) \in H\right)$, $F=\left([s, T] \ni t \mapsto X_{t}^{n}(\omega) \in H\right)$ for $n \in \mathbb{N}, \omega \in \Omega$ in the notation of [19, Corollary 2.7]) prove that for every $r \in(0, \infty), n \in \mathbb{N}, \omega \in \Omega, t \in\left[s, \tau_{r}^{n}(\omega)\right]$ it holds that

$$
\begin{aligned}
\left\|X_{t}^{n}(\omega)\right\|_{H}^{2} e^{-\alpha_{t}(1, \omega)} \\
=\|\xi(\omega)\|_{H}^{2}+\int_{s}^{t} e^{-\alpha_{u}(1, \omega)}\left[2\left\langle X_{u}^{n}(\omega), f\left(u, X_{\kappa(n, u)}^{n}(\omega), \omega\right)\right\rangle_{H}-\mathcal{K}_{u}(1, \omega)\left\|X_{u}^{n}(\omega)\right\|_{H}^{2}\right] d u \\
=\|\xi(\omega)\|_{H}^{2}+\int_{s}^{t} e^{-\alpha_{u}(1, \omega)}\left[2\left\langle X_{\kappa(n, u)}^{n}(\omega), f\left(u, X_{\kappa(n, u)}^{n}(\omega), \omega\right)\right\rangle_{H}\right. \\
\left.\quad-2\left\langle p_{u}^{n}(\omega), f\left(u, X_{\kappa(n, u)}^{n}(\omega), \omega\right)\right\rangle_{H}-\mathcal{K}_{u}(1, \omega)\left\|X_{u}^{n}(\omega)\right\|_{H}^{2}\right] d u .
\end{aligned}
$$

Combining this, the assumption that for every $t \in[s, T], x \in H, \omega \in \Omega$ it holds that $2\langle x, f(t, x, \omega)\rangle_{H} \leq K_{t}(1, \omega)\left(1+\|x\|_{H}^{2}\right)$, the Cauchy-Schwarz inequality, and (24) implies that for every $r \in(0, \infty), n \in \mathbb{N}, \omega \in \Omega, t \in\left[s, \tau_{r}^{n}(\omega)\right]$ it holds that

$$
\begin{aligned}
& \left\|X_{t}^{n}(\omega)\right\|_{H}^{2} e^{-\alpha_{t}(1, \omega)} \leq\|\xi(\omega)\|_{H}^{2} \\
& \quad+\int_{s}^{t} e^{-\alpha_{u}(1, \omega)}\left[\mathcal{K}_{u}(1, \omega)\left(1+\left\|X_{\kappa(n, u)}^{n}(\omega)\right\|_{H}^{2}\right)+2\left\|p_{u}^{n}(\omega)\right\|_{H}\left\|f\left(u, X_{\kappa(n, u)}^{n}(\omega), \omega\right)\right\|_{H}\right] d u \\
& \leq\|\xi(\omega)\|_{H}^{2}+\int_{s}^{t} e^{-\alpha_{u}(1, \omega)}\left[\mathcal{K}_{u}(1, \omega)\left(1+\left\|X_{\kappa(n, u)}^{n}(\omega)\right\|_{H}^{2}\right)+2 \mathcal{K}_{u}(r, \omega)\left\|p_{u}^{n}(\omega)\right\|_{H}\right] d u .
\end{aligned}
$$

The fact that for every $r \in(0, \infty), n \in \mathbb{N}, \omega \in \Omega, u \in[s, T]$ it holds that $\mathbb{1}_{\left[s, \tau_{r}^{n}(\omega)\right]}(u)\left\|X_{u}^{n}(\omega)\right\|_{H}^{2} \leq$ 
$r^{2}$, Fatou's Lemma, and (23) hence assure that for every $r \in(0, \infty), \omega \in \Omega, t \in[s, T]$ it holds that

$$
\begin{aligned}
& \lim \sup _{n \rightarrow \infty} \sup _{u \in[s, t]}\left(\mathbb{1}_{\left[s, \tau_{r}^{n}(\omega)\right]}(u)\left\|X_{u}^{n}(\omega)\right\|_{H}^{2}\right) e^{-\alpha_{t}(1, \omega)} \\
& \leq\|\xi(\omega)\|_{H}^{2}+\int_{s}^{t} e^{-\alpha_{u}(1, \omega)} \mathcal{K}_{u}(1, \omega)\left[1+\lim \sup _{n \rightarrow \infty} \sup _{v \in[s, u]}\left(\mathbb{1}_{\left[s, \tau_{r}^{n}(\omega)\right]}(v)\left\|X_{v}^{n}(\omega)\right\|_{H}^{2}\right)\right] d u \\
&+2 \lim \sup _{n \rightarrow \infty} \int_{s}^{t} \mathbb{1}_{\left[s, \tau_{r}^{n}(\omega)\right]}(u) \mathcal{K}_{u}(r, \omega)\left\|p_{u}^{n}(\omega)\right\|_{H} d u \\
&=\|\xi(\omega)\|_{H}^{2}+\int_{s}^{t} e^{-\alpha_{u}(1, \omega)} \mathcal{K}_{u}(1, \omega) d u \\
&+\int_{s}^{t} \mathcal{K}_{u}(1, \omega) \lim \sup _{n \rightarrow \infty} \sup _{v \in[s, u]}\left(\mathbb{1}_{\left[s, \tau_{r}^{n}(\omega)\right]}(v)\left\|X_{v}^{n}(\omega)\right\|_{H}^{2}\right) e^{-\alpha_{u}(1, \omega)} d u
\end{aligned}
$$

Gronwall's lemma therefore demonstrates that for every $r \in(0, \infty), \omega \in \Omega, t \in[s, T]$ it holds that

$$
\begin{aligned}
& \lim \sup _{n \rightarrow \infty} \sup _{u \in[s, t]}\left(\mathbb{1}_{\left[s, \tau_{r}^{n}(\omega)\right]}(u)\left\|X_{u}^{n}(\omega)\right\|_{H}^{2}\right) e^{-\alpha_{t}(1, \omega)} \\
& \leq\left[\|\xi(\omega)\|_{H}^{2}+\int_{s}^{t} e^{-\alpha_{u}(1, \omega)} \mathcal{K}_{u}(1, \omega) d u\right] \exp \left(\int_{s}^{t} \mathcal{K}_{u}(1, \omega) d u\right) .
\end{aligned}
$$

The change of variables formula hence establishes that for every $r \in(0, \infty), \omega \in \Omega, t \in[s, T]$ it holds that

$$
\begin{aligned}
& \lim \sup _{n \rightarrow \infty} \sup _{u \in[s, t]}\left(\mathbb{1}_{\left[s, \tau_{r}^{n}(\omega)\right]}(u)\left\|X_{u}^{n}(\omega)\right\|_{H}^{2}\right) \\
& \leq e^{2 \alpha_{t}(1, \omega)}\left[\|\xi(\omega)\|_{H}^{2}+\int_{\alpha_{s}(1, \omega)}^{\alpha_{t}(1, \omega)} e^{-v} d v\right] \leq e^{2 \alpha_{t}(1, \omega)}\left[\|\xi(\omega)\|_{H}^{2}+1\right] .
\end{aligned}
$$

This shows for every $r \in(0, \infty), \omega \in \Omega$ that

$$
\lim \sup _{n \rightarrow \infty} \sup _{u \in[s, T]}\left(\mathbb{1}_{\left[s, \tau_{r}^{n}(\omega)\right]}(u)\left\|X_{u}^{n}(\omega)\right\|_{H}^{2}\right) \leq e^{2 \alpha_{T}(1, \omega)}\left[\|\xi(\omega)\|_{H}^{2}+1\right] .
$$

Therefore, we obtain that there exist functions $N: \Omega \rightarrow \mathbb{N}$ and $M: \Omega \rightarrow(0, \infty)$ which satisfy that for every $\omega \in \Omega, n \in[N(\omega), \infty) \cap \mathbb{N}$ it holds that $M(\omega)=1+e^{\alpha_{T}(1, \omega)} \sqrt{\|\xi(\omega)\|_{H}^{2}+1}$ and

$$
\sup _{u \in[s, T]}\left(\mathbb{1}_{\left[s, \tau_{M(\omega)}^{n}(\omega)\right]}(u)\left\|X_{u}^{n}(\omega)\right\|_{H}^{2}\right) \leq[M(\omega)-1]^{2}+1<[M(\omega)]^{2} .
$$

Note that (31) shows that for every $\omega \in \Omega, n \in[N(\omega), \infty) \cap \mathbb{N}$ it holds that $\tau_{M(\omega)}^{n}(\omega)=T$ and

$$
\sup _{u \in[s, T]}\left\|X_{u}^{n}(\omega)\right\|_{H} \leq \sqrt{[M(\omega)-1]^{2}+1} \leq M(\omega) .
$$

Furthermore, note that for every $t \in[s, T], r \in(0, \infty), \omega \in \Omega, m, n \in \mathbb{N}$ it holds that

$$
\begin{aligned}
& \left\|X_{t}^{n}(\omega)-X_{t}^{m}(\omega)\right\|_{H}^{2} e^{-2 \alpha_{t}(r, \omega)} \\
& =2 \int_{s}^{t}\left[\left\langle X_{u}^{n}(\omega)-X_{u}^{m}(\omega), f\left(u, X_{u}^{n}(\omega)+p_{u}^{n}(\omega), \omega\right)-f\left(u, X_{u}^{m}(\omega)+p_{u}^{m}(\omega), \omega\right)\right\rangle_{H}\right. \\
& \left.-\mathcal{K}_{u}(r, \omega)\left\|X_{u}^{n}(\omega)-X_{u}^{m}(\omega)\right\|_{H}^{2}\right] e^{-2 \alpha_{u}(r, \omega)} d u \\
& =2 \int_{s}^{t}\left[\left\langle p_{u}^{m}(\omega)-p_{u}^{n}(\omega), f\left(u, X_{u}^{n}(\omega)+p_{u}^{n}(\omega), \omega\right)-f\left(u, X_{u}^{m}(\omega)+p_{u}^{m}(\omega), \omega\right)\right\rangle_{H}\right. \\
& +\left\langle X_{u}^{n}(\omega)+p_{u}^{n}(\omega)-X_{u}^{m}(\omega)-p_{u}^{m}(\omega), f\left(u, X_{u}^{n}(\omega)+p_{u}^{n}(\omega), \omega\right)-f\left(u, X_{u}^{m}(\omega)+p_{u}^{m}(\omega), \omega\right)\right\rangle_{H} \\
& \left.-\mathcal{K}_{u}(r, \omega)\left\|X_{u}^{n}(\omega)-X_{u}^{m}(\omega)\right\|_{H}^{2}\right] e^{-2 \alpha_{u}(r, \omega)} d u .
\end{aligned}
$$


Combining (13), (32), and the Cauchy-Schwarz inequality hence ensures that for every $t \in[s, T]$, $\omega \in \Omega, m, n \in[N(\omega), \infty) \cap \mathbb{N}$ it holds that

$$
\begin{aligned}
& \left\|X_{t}^{n}(\omega)-X_{t}^{m}(\omega)\right\|_{H}^{2} e^{-2 \alpha_{t}(M(\omega), \omega)} \leq \int_{s}^{t} e^{-2 \alpha_{u}(M(\omega), \omega)} \\
& \quad \cdot\left[2\left\langle p_{u}^{m}(\omega)-p_{u}^{n}(\omega), f\left(u, X_{u}^{n}(\omega)+p_{u}^{n}(\omega), \omega\right)-f\left(u, X_{u}^{m}(\omega)+p_{u}^{m}(\omega), \omega\right)\right\rangle_{H}\right. \\
& \quad+\mathcal{K}_{u}(M(\omega), \omega)\left\|\left(X_{u}^{n}(\omega)-X_{u}^{m}(\omega)\right)+\left(p_{u}^{n}(\omega)-p_{u}^{m}(\omega)\right)\right\|_{H}^{2} \\
& \left.\quad-2 \mathcal{K}_{u}(M(\omega), \omega)\left\|X_{u}^{n}(\omega)-X_{u}^{m}(\omega)\right\|_{H}^{2}\right] d u \\
& \leq \int_{s}^{t} e^{-2 \alpha_{u}(M(\omega), \omega)}\left[2\left\|p_{u}^{m}(\omega)-p_{u}^{n}(\omega)\right\|_{H}\left\|f\left(u, X_{u}^{n}(\omega)+p_{u}^{n}(\omega), \omega\right)-f\left(u, X_{u}^{m}(\omega)+p_{u}^{m}(\omega), \omega\right)\right\|_{H}\right. \\
& \quad+2 \mathcal{K}_{u}(M(\omega), \omega)\left(\left\|X_{u}^{n}(\omega)-X_{u}^{m}(\omega)\right\|_{H}^{2}+\left\|p_{u}^{n}(\omega)-p_{u}^{m}(\omega)\right\|_{H}^{2}\right) \\
& \left.\quad-2 \mathcal{K}_{u}(M(\omega), \omega)\left\|X_{u}^{n}(\omega)-X_{u}^{m}(\omega)\right\|_{H}^{2}\right] d u .
\end{aligned}
$$

This implies for every $t \in[s, T], \omega \in \Omega, m, n \in[N(\omega), \infty) \cap \mathbb{N}$ that

$$
\begin{aligned}
& \left\|X_{t}^{n}(\omega)-X_{t}^{m}(\omega)\right\|_{H}^{2} e^{-2 \alpha_{t}(M(\omega), \omega)} \\
& \leq 2 \int_{s}^{t} e^{-2 \alpha_{u}(M(\omega), \omega)}\left[2\left\|p_{u}^{m}(\omega)-p_{u}^{n}(\omega)\right\|_{H} L_{M(\omega)}(\omega)+\mathcal{K}_{u}(M(\omega), \omega)\left\|p_{u}^{n}(\omega)-p_{u}^{m}(\omega)\right\|_{H}^{2}\right] d u \\
& \leq 2 \int_{s}^{t} e^{-2 \alpha_{u}(M(\omega), \omega)} \mathcal{K}_{u}(M(\omega), \omega)\left[2\left\|p_{u}^{m}(\omega)-p_{u}^{n}(\omega)\right\|_{H}+\left\|p_{u}^{m}(\omega)-p_{u}^{n}(\omega)\right\|_{H}^{2}\right] d u
\end{aligned}
$$

Moreover, note that (32) establishes for every $u \in[s, T], \omega \in \Omega, m, n \in[N(\omega), \infty) \cap \mathbb{N}$ that

$$
\left\|p_{u}^{m}(\omega)-p_{u}^{n}(\omega)\right\|_{H}^{2} \leq 4 M(\omega)\left(\left\|p_{u}^{m}(\omega)\right\|_{H}+\left\|p_{u}^{n}(\omega)\right\|_{H}\right) .
$$

Combining this and (35) shows that for every $t \in[s, T], \omega \in \Omega, m, n \in[N(\omega), \infty) \cap \mathbb{N}$ it holds that

$$
\begin{aligned}
& \left\|X_{t}^{n}(\omega)-X_{t}^{m}(\omega)\right\|_{H}^{2} e^{-2 \alpha_{t}(M(\omega), \omega)} \\
& \leq 4(1+2 M(\omega))\left(\int_{s}^{T} \mathcal{K}_{u}(M(\omega), \omega)\left(\left\|p_{u}^{n}(\omega)\right\|_{H}+\left\|p_{u}^{m}(\omega)\right\|_{H}\right) d u\right) .
\end{aligned}
$$

In addition, observe that the fact that for every $\omega \in \Omega, n \in[N(\omega), \infty) \cap \mathbb{N}$ it holds that $\tau_{M(\omega)}^{n}(\omega)=$ $T$, (23), and (32) assure that for every $\omega \in \Omega$ it holds that

$$
\begin{aligned}
& \lim \sup _{n \rightarrow \infty} \int_{s}^{T}\left\|p_{u}^{n}(\omega)\right\|_{H} \mathcal{K}_{u}(M(\omega), \omega) d u \\
& =\lim \sup _{n \rightarrow \infty} \int_{s}^{T} \mathbb{1}_{\left[s, \tau_{M(\omega)}^{n}(\omega)\right]}(u)\left\|p_{u}^{n}(\omega)\right\|_{H} \mathcal{K}_{u}(M(\omega), \omega) d u=0 .
\end{aligned}
$$

This and (37) demonstrate that for every $\omega \in \Omega$ it holds that $\left([s, T] \ni t \mapsto X_{t}^{n}(\omega) \in H\right) \in$ $\mathcal{C}([s, T], H), n \in \mathbb{N}$, is a Cauchy sequence. The fact that the space $\mathcal{C}([s, T], H)$ with the supremum norm is complete hence ensures that there exists a function $X:[s, T] \times \Omega \rightarrow H$ which satisfies for every $\omega \in \Omega$ that $\left([s, T] \ni t \mapsto X_{t}(\omega) \in H\right) \in \mathcal{C}([s, T], H)$ and

$$
\lim \sup _{n \rightarrow \infty} \sup _{t \in[s, T]}\left\|X_{t}^{n}(\omega)-X_{t}(\omega)\right\|_{H}=0 .
$$


Observe that the assumption that for every $\omega \in \Omega$ it holds that $([s, T] \times H \ni(t, x) \mapsto$ $f(t, x, \omega) \in H) \in \mathcal{C}([s, T] \times H, H)$, the assumption that for every $r \in(0, \infty), \omega \in \Omega$ it holds that $\sup _{t \in[s, T]} \sup _{x \in H,\|x\|_{H} \leq r}\|f(t, x, \omega)\|_{H}<\infty$, (32), (39), and the dominated convergence theorem prove that for every $t \in[s, T], \omega \in \Omega$ it holds that

$$
\begin{aligned}
& \limsup _{n \rightarrow \infty}\left\|\int_{s}^{t} f\left(u, X_{u}^{n}(\omega), \omega\right) d u-\int_{s}^{t} f\left(u, X_{u}(\omega), \omega\right) d u\right\|_{H} \\
& \leq \lim \sup _{n \rightarrow \infty} \int_{s}^{t}\left\|f\left(u, X_{u}^{n}(\omega), \omega\right)-f\left(u, X_{u}(\omega), \omega\right)\right\|_{H} d u \\
& =\int_{s}^{t} \lim \sup _{n \rightarrow \infty}\left\|f\left(u, X_{u}^{n}(\omega), \omega\right)-f\left(u, X_{u}(\omega), \omega\right)\right\|_{H} d u=0 .
\end{aligned}
$$

Moreover, observe that (39) assures that for every $\omega \in \Omega$ it holds that the sequence $X^{n}(\omega) \in$ $\mathcal{C}([s, T], H), n \in \mathbb{N}$, is uniformly equicontinuous. This implies for every $\omega \in \Omega$ that

$$
\lim \sup _{n \rightarrow \infty} \sup _{u \in[s, T]}\left\|X_{\kappa(n, u)}^{n}(\omega)-X_{u}^{n}(\omega)\right\|_{H}=0 .
$$

The assumption that for every $\omega \in \Omega$ it holds that $([s, T] \times H \ni(t, x) \mapsto f(t, x, \omega) \in$ $H) \in \mathcal{C}([s, T] \times H, H)$, the assumption that for every $r \in(0, \infty), \omega \in \Omega$ it holds that $\sup _{t \in[s, T]} \sup _{x \in H,\|x\|_{H} \leq r}\|f(t, x, \omega)\|_{H}<\infty$, (32), and the dominated convergence theorem therefore show that for every $t \in[s, T], \omega \in \Omega$ it holds that

$$
\begin{aligned}
& \lim \sup _{n \rightarrow \infty}\left\|\int_{s}^{t} f\left(u, X_{\kappa(n, u)}^{n}(\omega), \omega\right) d u-\int_{s}^{t} f\left(u, X_{u}^{n}(\omega), \omega\right) d u\right\|_{H} \\
& \leq \lim \sup _{n \rightarrow \infty} \int_{s}^{t}\left\|f\left(u, X_{\kappa(n, u)}^{n}(\omega), \omega\right)-f\left(u, X_{u}^{n}(\omega), \omega\right)\right\|_{H} d u \\
& \leq \int_{s}^{t} \lim \sup _{n \rightarrow \infty}\left\|f\left(u, X_{\kappa(n, u)}^{n}(\omega), \omega\right)-f\left(u, X_{u}^{n}(\omega), \omega\right)\right\|_{H} d u=0 .
\end{aligned}
$$

The triangle inequality and (40) hence ensure that for every $t \in[s, T], \omega \in \Omega$ it holds that

$$
\lim \sup _{n \rightarrow \infty}\left\|\int_{s}^{t} f\left(u, X_{\kappa(n, u)}^{n}(\omega), \omega\right) d u-\int_{s}^{t} f\left(u, X_{u}(\omega), \omega\right) d u\right\|_{H}=0 .
$$

Combining this, (24), and (39) implies that for every $t \in[s, T], \omega \in \Omega$ it holds that

$$
X_{t}(\omega)=\xi(\omega)+\int_{s}^{t} f\left(u, X_{u}(\omega), \omega\right) d u
$$

Next note that (13) proves that for every function $\mathbf{X}:[s, T] \times \Omega \rightarrow H$ with $\forall \omega \in \Omega:([s, T] \ni t \mapsto$ $\left.\mathbf{X}_{t}(\omega) \in H\right) \in \mathcal{C}([s, T], H)$ and $\forall t \in[s, T], \omega \in \Omega: \mathbf{X}_{t}(\omega)=\xi(\omega)+\int_{s}^{t} f\left(u, \mathbf{X}_{u}(\omega), \omega\right) d u$ and every $t \in[s, T], \omega \in \Omega, r \in\left(\sup _{u \in[s, T]} \max \left\{\left\|X_{u}(\omega)\right\|_{H},\left\|\mathbf{X}_{u}(\omega)\right\|_{H}\right\}, \infty\right)$ it holds that

$$
\begin{aligned}
e^{-2 \alpha_{t}(r, \omega)}\left\|X_{t}(\omega)-\mathbf{X}_{t}(\omega)\right\|_{H}^{2} \\
=2 \int_{s}^{t} e^{-2 \alpha_{u}(r, \omega)}\left[\left\langle X_{u}(\omega)-\mathbf{X}_{u}(\omega), f\left(u, X_{u}(\omega), \omega\right)-f\left(u, \mathbf{X}_{u}(\omega), \omega\right)\right\rangle_{H}\right. \\
\left.\quad-\mathcal{K}_{u}(r, \omega)\left\|X_{u}(\omega)-\mathbf{X}_{u}(\omega)\right\|_{H}^{2}\right] d u \\
\leq \int_{s}^{t} \mathcal{K}_{u}(r, \omega) e^{-2 \alpha_{u}(r, \omega)}\left\|X_{u}(\omega)-\mathbf{X}_{u}(\omega)\right\|_{H}^{2} d u \\
\leq \sup _{u \in[s, T]}\left|\mathcal{K}_{u}(r, \omega)\right| \int_{s}^{t} e^{-2 \alpha_{u}(r, \omega)}\left\|X_{u}(\omega)-\mathbf{X}_{u}(\omega)\right\|_{H}^{2} d u<\infty
\end{aligned}
$$


Gronwall's lemma hence implies that for every function $\mathbf{X}:[s, T] \times \Omega \rightarrow H$ with $\forall \omega \in \Omega:([s, T] \ni$ $\left.t \mapsto \mathbf{X}_{t}(\omega) \in H\right) \in \mathcal{C}([s, T], H)$ and $\forall t \in[s, T], \omega \in \Omega: \mathbf{X}_{t}(\omega)=\xi(\omega)+\int_{s}^{t} f\left(u, \mathbf{X}_{u}(\omega), \omega\right) d u$ and every $t \in[s, T], \omega \in \Omega$ it holds that

$$
X_{t}(\omega)=\mathbf{X}_{t}(\omega) .
$$

Combining this and (44) establishes item (ii). In addition, note that the assumption that for every $\omega \in \Omega$ it holds that $([s, T] \times H \ni(t, x) \mapsto f(t, x, \omega) \in H) \in \mathcal{C}([s, T] \times H, H)$, the assumption that for every $t \in[s, T], u \in[s, t], x \in H$ it holds that $\Omega \ni \omega \mapsto f(u, x, \omega) \in H$ is $\mathbb{F}_{t} / \mathcal{B}(H)$ measurable, and Lemma 2.1 (with $(\Omega, \mathcal{F})=\left(\Omega, \mathbb{F}_{t}\right), X=[s, T] \times H, d_{X}=([s, T] \times H \times[s, T] \times H \ni$ $\left.\left(t_{1}, x_{1}, t_{2}, x_{2}\right) \mapsto\left|t_{1}-t_{2}\right|+\left\|x_{1}-x_{2}\right\|_{H} \in[0, \infty)\right), Y=H, d_{Y}=\left(H \times H \ni\left(x_{1}, x_{2}\right) \mapsto\left\|x_{1}-x_{2}\right\|_{H} \in\right.$ $[0, \infty)), f=([s, t] \times H \times \Omega \ni(u, x, \omega) \mapsto f(u, x, \omega) \in H)$ for $t \in[s, T]$ in the notation of Lemma 2.1) show that for every $t \in[s, T]$ it holds that

$$
[s, t] \times H \times \Omega \ni(u, x, \omega) \mapsto f(u, x, \omega) \in H
$$

is $\left(\mathcal{B}([s, t]) \otimes \mathcal{B}(H) \otimes \mathbb{F}_{t}\right) / \mathcal{B}(H)$-measurable. The fact that for every $t \in[s, T]$ and every $\mathbb{F}_{t} / \mathcal{B}(H)$ measurable function $\zeta: \Omega \rightarrow H$ it holds that $[s, t] \times \Omega \ni(u, \omega) \mapsto(u, \zeta(\omega), \omega) \in[s, t] \times H \times \Omega$ is $\left(\mathcal{B}([s, t]) \otimes \mathbb{F}_{t}\right) /\left(\mathcal{B}([s, t]) \otimes \mathcal{B}(H) \otimes \mathbb{F}_{t}\right)$-measurable hence assures that for every $t \in[s, T]$ and every $\mathbb{F}_{t} / \mathcal{B}(H)$-measurable function $\zeta: \Omega \rightarrow H$ it holds that

$$
[s, t] \times \Omega \ni(u, \omega) \mapsto f(u, \zeta(\omega), \omega) \in H
$$

is $\left(\mathcal{B}([s, t]) \otimes \mathbb{F}_{t}\right) / \mathcal{B}(H)$-measurable. The assumption that $\xi: \Omega \rightarrow H$ is $\mathbb{F}_{s} / \mathcal{B}(H)$-measurable, (15)), and Lemma 2.2 (with $X=H, \Omega=\Omega, \mathcal{F}=\mathbb{F}_{t}, a=s+(k(T-s) / n), b=t, f=([s+(k(T-s) / n), t] \times \Omega \ni$ $\left.(u, \omega) \mapsto f\left(u, X_{s+(k(T-s) / n)}^{n}(\omega), \omega\right) \in H\right), F=\left(\Omega \ni \omega \rightarrow \int_{s+(k(T-s) / n)}^{t} f\left(u, X_{s+(k(T-s) / n)}^{n}(\omega), \omega\right) d u \in\right.$ $H)$ for $t \in(s+(k(T-s) / n), s+((k+1)(T-s) / n)], k \in\{0,1, \ldots, n-1\}, n \in \mathbb{N}$ in the notation of Lemma 2.2) therefore imply that for every $n \in \mathbb{N}$ it holds that $\left(X_{t}^{n}\right)_{t \in[s, T]}$ is $\left(\mathbb{F}_{t}\right)_{t \in[s, T] \text {-adapted. }}$ Combining this and (39) establishes item (iii). The proof of Lemma 2.3 is thus completed.

Corollary 2.4. Assume Setting [1.2, assume that $\operatorname{dim}(H)<\infty$, let $T \in(0, \infty), s \in[0, T]$, $C, c \in[0, \infty), \delta, \kappa \in \mathbb{R}, F \in \mathcal{C}(H, H), \Phi \in \mathcal{C}(H,[0, \infty))$, let $\left(\Omega, \mathcal{F}, \mathbb{P},\left(\mathbb{F}_{t}\right)_{t \in[0, T]}\right)$ be a filtered probability space, let $\xi \in \mathcal{M}\left(\mathbb{F}_{s}, \mathcal{B}(H)\right)$, let $O:[0, T] \times \Omega \rightarrow H$ be an $\left(\mathbb{F}_{t}\right)_{t \in[0, T]}$-adapted stochastic process with continuous sample paths, and assume for every $x, y \in H$ that $\|F(x)-F(y)\|_{H} \leq$ $C\|x-y\|_{H_{\delta}}\left(1+\|x\|_{H_{\kappa}}^{c}+\|y\|_{H_{\kappa}}^{c}\right)$ and $\langle x, A x+F(x+y)\rangle_{H} \leq \Phi(y)\left(1+\|x\|_{H}^{2}\right)$. Then

(i) there exists a unique function $X:[s, T] \times \Omega \rightarrow H$ which satisfies for every $t \in[s, T], \omega \in \Omega$ that $\left([s, T] \ni u \mapsto X_{u}(\omega) \in H\right) \in \mathcal{C}([s, T], H)$ and

$$
X_{t}(\omega)=e^{(t-s) A} \xi(\omega)+\int_{s}^{t} e^{(t-u) A} F\left(X_{u}(\omega)\right) d u+O_{t}(\omega)-e^{(t-s) A} O_{s}(\omega)
$$

and

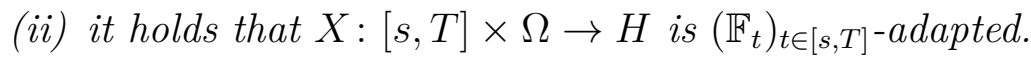

Proof of Corollary 2.4. Throughout this proof let $K:(0, \infty) \times \Omega \rightarrow[0, \infty)$ be the function which satisfies for every $r \in(0, \infty), \omega \in \Omega$ that $K(r, \omega)=\max \left\{C\left\|(-A)^{\delta}\right\|_{L(H)} \max \left\{\left\|(-A)^{\kappa}\right\|_{L(H)}^{c}, 1\right\}(1+\right.$ $\left.\left.2\left(r+\sup _{u \in[0, T]}\left\|O_{u}(\omega)\right\|_{H}\right)^{c}\right), \sup _{u \in[0, T]} \Phi\left(O_{u}(\omega)\right)\right\}$. Note that the assumption that for every $x, y \in$ 
$H$ it holds that $\|F(x)-F(y)\|_{H} \leq C\|x-y\|_{H_{\delta}}\left(1+\|x\|_{H_{\kappa}}^{c}+\|y\|_{H_{\kappa}}^{c}\right)$ implies that for every $t \in[0, T]$, $x, y \in H, r \in(0, \infty), \omega \in \Omega$ with $\min \left\{\|x\|_{H},\|y\|_{H}\right\} \leq r$ it holds that

$$
\begin{aligned}
& \left\langle x-y, A(x-y)+F\left(x+O_{t}(\omega)\right)-F\left(y+O_{t}(\omega)\right)\right\rangle_{H} \\
& \leq\langle x-y, A(x-y)\rangle_{H}+\|x-y\|_{H}\left\|F\left(x+O_{t}(\omega)\right)-F\left(y+O_{t}(\omega)\right)\right\|_{H} \\
& \leq C\|x-y\|_{H}\|x-y\|_{H_{\delta}}\left(1+\left\|x+O_{t}(\omega)\right\|_{H_{\kappa}}^{c}+\left\|y+O_{t}(\omega)\right\|_{H_{\kappa}}^{c}\right) \\
& \leq C\left\|(-A)^{\delta}\right\|_{L(H)} \max \left\{\left\|(-A)^{\kappa}\right\|_{L(H)}^{c}, 1\right\}\left(1+2\left(r+\sup _{u \in[0, T]}\left\|O_{u}(\omega)\right\|_{H}\right)^{c}\right)\|x-y\|_{H}^{2} \\
& \leq K(r, \omega)\|x-y\|_{H}^{2}<\infty .
\end{aligned}
$$

In addition, observe that the assumption that for every $x, y \in H$ it holds that $\langle x, A x+F(x+y)\rangle_{H} \leq$ $\Phi(y)\left(1+\|x\|_{H}^{2}\right)$ shows that for every $t \in[0, T], x, y \in H, \omega \in \Omega$ it holds that

$$
\left\langle x, A x+F\left(x+O_{t}(\omega)\right)\right\rangle_{H} \leq \Phi\left(O_{t}(\omega)\right)\left(1+\|x\|_{H}^{2}\right) \leq \sup _{u \in[0, T]} \Phi\left(O_{u}(\omega)\right)\left(1+\|x\|_{H}^{2}\right) .
$$

Moreover, note that the assumption that $\operatorname{dim}(H)<\infty$, the assumption that $F \in \mathcal{C}(H, H)$, and the assumption that $O:[0, T] \times \Omega \rightarrow H$ has continuous sample paths ensure that for every $r \in(0, \infty)$, $\omega \in \Omega$ it holds that $\left([s, T] \times H \ni(u, x) \mapsto\left(A x+F\left(x+O_{u}(\omega)\right)\right) \in H\right) \in \mathcal{C}([s, T] \times H, H)$ and

$$
\sup _{u \in[s, T]} \sup _{x \in H,\|x\|_{H} \leq r}\left\|A x+F\left(x+O_{u}(\omega)\right)\right\|_{H}<\infty .
$$

The assumption that $\left(O_{t}\right)_{t \in[0, T]}$ is $\left(\mathbb{F}_{t}\right)_{t \in[0, T]}$-adapted, (50), (51), and Lemma 2.3 (with $H=H$, $T=T, s=s,\left(\Omega, \mathcal{F}, \mathbb{P},\left(\mathbb{F}_{u}\right)_{u \in[s, T]}\right)=\left(\Omega, \mathcal{F}, \mathbb{P},\left(\mathbb{F}_{u}\right)_{u \in[s, T]}\right), \xi=\xi-O_{s}, f=([s, T] \times H \times \Omega \ni$ $\left.(u, h, \omega) \mapsto A h+F\left(h+O_{u}(\omega)\right) \in H\right), K_{t}(r, \omega)=2 K(r, \omega)$ for $t \in[s, T], r \in(0, \infty)$ in the notation of Lemma 2.3) therefore prove that

(a) there exists a unique function $\mathcal{X}:[s, T] \times \Omega \rightarrow H$ which satisfies for every $t \in[s, T], \omega \in \Omega$ that $\left([s, T] \ni u \mapsto \mathcal{X}_{u}(\omega) \in H\right) \in \mathcal{C}([s, T], H)$ and

$$
\mathcal{X}_{t}(\omega)=\xi(\omega)-O_{s}(\omega)+\int_{s}^{t}\left[A \mathcal{X}_{u}(\omega)+F\left(\mathcal{X}_{u}(\omega)+O_{u}(\omega)\right)\right] d u
$$

and

(b) it holds that $\mathcal{X}:[s, T] \times \Omega \rightarrow H$ is $\left(\mathbb{F}_{t}\right)_{t \in[s, T] \text {-adapted. }}$

Next let $X:[s, T] \times \Omega \rightarrow H$ be the stochastic process with continuous sample paths which satisfies for every $t \in[s, T], \omega \in \Omega$ that

$$
X_{t}(\omega)=\mathcal{X}_{t}(\omega)+O_{t}(\omega)
$$

In addition, observe that (53) implies for every $t \in[s, T], \omega \in \Omega$ that

$$
\mathcal{X}_{t}(\omega)=e^{(t-s) A}\left(\xi(\omega)-O_{s}(\omega)\right)+\int_{s}^{t} e^{(t-u) A} F\left(\mathcal{X}_{u}(\omega)+O_{u}(\omega)\right) d u .
$$

This and (54) show that for every $t \in[s, T], \omega \in \Omega$ it holds that

$$
X_{t}(\omega)=e^{(t-s) A} \xi(\omega)+\int_{s}^{t} e^{(t-u) A} F\left(X_{u}(\omega)\right) d u+O_{t}(\omega)-e^{(t-s) A} O_{s}(\omega) .
$$

Moreover, observe that for every function $Y:[s, T] \times \Omega \rightarrow H$ with $\forall \omega \in \Omega:([s, T] \ni t \mapsto$ $\left.Y_{t}(\omega)\right) \in \mathcal{C}([s, T], H)$ and $\forall t \in[s, T], \omega \in \Omega: Y_{t}(\omega)=e^{(t-s) A} \xi(\omega)+\int_{s}^{t} e^{(t-u) A} F\left(Y_{u}(\omega)\right) d u+$ $O_{t}(\omega)-e^{(t-s) A} O_{s}(\omega)$ and every $t \in[s, T], \omega \in \Omega$ it holds that $Y_{t}(\omega)-O_{t}(\omega)=\xi(\omega)-O_{s}(\omega)+$ $\int_{s}^{t}\left[A\left(Y_{u}(\omega)-O_{u}(\omega)\right)+F\left(\left[Y_{u}(\omega)-O_{u}(\omega)\right]+O_{u}(\omega)\right)\right] d u$. The fact that for every $\omega \in \Omega$ it holds that $\left([s, T] \ni t \mapsto\left[X_{t}(\omega)-O_{t}(\omega)\right] \in H\right) \in \mathcal{C}([s, T], H)$, item (国), and (56) therefore establish item (ii). Furthermore, note that item (b) , the fact that $\left(O_{t}\right)_{t \in[0, T]}$ is $\left(\mathbb{F}_{t}\right)_{t \in[0, T]}$-adapted, and (54) establish item (iii). The proof of Corollary 2.4 is thus completed. 


\section{Strong a priori bounds based on bootstrap-type argu- ments}

In this section we provide in Lemmas 3.2 3.4 appropriate a priori bounds for the approximation process $\left(Y_{t}\right)_{t \in[0, T]}$ introduced in Setting 3.1 below. The considered equations can, in particular, be thought of as discretizations in space and time of an underlying stochastic Burgers equation. The proofs of Lemmas 3.23 .4 are based on suitable bootstrap-type arguments, which have been intensively used in the literature to establish regularity properties of solutions to (stochastic) evolution equations (cf., e.g., [21, 22] and the references mentioned therein).

Setting 3.1. Assume Setting 1.2, let $(\Omega, \mathcal{F}, \mathbb{P})$ be a probability space, let $T \in(0, \infty), \beta \in$ $[0,1), \gamma \in[0, \beta], \xi \in \mathcal{M}\left(\mathcal{F}, \mathcal{B}\left(H_{\beta}\right)\right), \quad F \in \mathcal{M}\left(\mathcal{B}\left(H_{\gamma}\right), \mathcal{B}(H)\right), \quad \kappa \in \mathcal{M}(\mathcal{B}([0, T]), \mathcal{B}([0, T]))$, $Z \in \mathcal{M}\left(\mathcal{B}([0, T]) \otimes \mathcal{F}, \mathcal{B}\left(H_{\gamma}\right)\right)$ satisfy for every $t \in[0, T]$ that $\kappa(t) \leq t$ and $\sup _{u \in[0, T]}\left\|Z_{u}\right\|_{H}+$ $\int_{0}^{t}\left\|e^{(t-\kappa(s)) A} F\left(Z_{s}\right)\right\|_{H} d s<\infty$, and let $O:[0, T] \times \Omega \rightarrow H_{\beta}$ and $Y:[0, T] \times \Omega \rightarrow H$ be stochastic processes with continuous sample paths which satisfy for every $t \in[0, T]$ that $Y_{t}=$ $e^{t A} \xi+\int_{0}^{t} e^{(t-\kappa(s)) A} F\left(Z_{s}\right) d s+O_{t}$.

Lemma 3.2. Assume Setting [3.1, let $p \in[1, \infty), \rho \in[0, \beta], \alpha \in[0,1-\rho)$, and assume that

$$
\left(\sup _{v \in H_{\gamma}} \frac{\|F(v)\|_{H_{-\alpha}}}{1+\|v\|_{H}^{2}}\right)<\infty .
$$

Then

(i) it holds for every $t \in[0, T]$ that $Y_{t}(\Omega) \subseteq H_{\rho}$,

(ii) it holds for every $t \in[0, T]$ that

$$
\left\|Y_{t}\right\|_{H_{\rho}} \leq\|\xi\|_{H_{\rho}}+\left\|O_{t}\right\|_{H_{\rho}}+\frac{T^{1-\alpha-\rho}}{1-\alpha-\rho}\left(\sup _{v \in H_{\gamma}} \frac{\|F(v)\|_{H_{-\alpha}}}{1+\|v\|_{H}^{2}}\right)\left(1+\sup _{u \in[0, T]}\left\|Z_{u}\right\|_{H}^{2}\right)<\infty
$$

and

(iii) it holds for every $t \in[0, T]$ that

$$
\begin{aligned}
\left\|Y_{t}\right\|_{\mathcal{L}^{p}\left(\mathbb{P} ; H_{\rho}\right)} \leq & \|\xi\|_{\mathcal{L}^{p}\left(\mathbb{P} ; H_{\rho}\right)}+\left\|O_{t}\right\|_{\mathcal{L}^{p}\left(\mathbb{P} ; H_{\rho}\right)} \\
& +\frac{T^{1-\alpha-\rho}}{1-\alpha-\rho}\left(\sup _{v \in H_{\gamma}} \frac{1+\|F(v)\|_{H_{-\alpha}}}{1+\|v\|_{H}^{2}}\right)\left(1+\sup _{u \in[0, T]}\left\|Z_{u}\right\|_{\mathcal{L}^{2 p}(\mathbb{P} ; H)}^{2}\right) .
\end{aligned}
$$

Proof of Lemma 3.2. Throughout this proof assume w.l.o.g. that $\sup _{v \in H_{\gamma}}\|F(v)\|_{H}>0$. Note that the assumption that $\forall t \in[0, T]: \kappa(t) \leq t$ implies that for every $t \in(0, T]$ it holds that

$$
\begin{aligned}
& \int_{0}^{t}\left\|e^{(t-\kappa(u)) A} F\left(Z_{u}\right)\right\|_{H_{\rho}} d u \leq \int_{0}^{t}\left\|(-A)^{\alpha+\rho} e^{(t-\kappa(u)) A}\right\|_{L(H)}\left\|F\left(Z_{u}\right)\right\|_{H_{-\alpha}} d u \\
& \leq \int_{0}^{t}(t-\kappa(u))^{-\alpha-\rho}\left\|F\left(Z_{u}\right)\right\|_{H_{-\alpha}} d u \\
& \leq\left(\sup _{v \in H_{\gamma}} \frac{\|F(v)\|_{H_{-\alpha}}}{1+\|v\|_{H}^{2}}\right) \int_{0}^{t}(t-\kappa(u))^{-\alpha-\rho}\left(1+\left\|Z_{u}\right\|_{H}^{2}\right) d u \\
& \leq\left(\sup _{v \in H_{\gamma}} \frac{\|F(v)\|_{H_{-\alpha}}}{1+\|v\|_{H}^{2}}\right) \int_{0}^{t}(t-u)^{-\alpha-\rho}\left(1+\left\|Z_{u}\right\|_{H}^{2}\right) d u
\end{aligned}
$$


Hence, we obtain that for every $t \in(0, T]$ it holds that

$$
\int_{0}^{t}\left\|e^{(t-\kappa(u)) A} F\left(Z_{u}\right)\right\|_{H_{\rho}} d u \leq \frac{t^{1-\alpha-\rho}}{1-\alpha-\rho}\left(\sup _{v \in H_{\gamma}} \frac{\|F(v)\|_{H_{-\alpha}}}{1+\|v\|_{H}^{2}}\right)\left(1+\sup _{u \in[0, T]}\left\|Z_{u}\right\|_{H}^{2}\right) .
$$

The triangle inequality, the assumption that $\sup _{u \in[0, T]}\left\|Z_{u}\right\|_{H}<\infty$, and (157) therefore prove that for every $t \in[0, T]$ it holds that $Y_{t}(\Omega) \subseteq H_{\rho}$ and

$$
\begin{aligned}
\left\|Y_{t}\right\|_{H_{\rho}} & \leq\|\xi\|_{H_{\rho}}+\int_{0}^{t}\left\|e^{(t-\kappa(u)) A} F\left(Z_{u}\right)\right\|_{H_{\rho}} d u+\left\|O_{t}\right\|_{H_{\rho}} \\
& \leq\|\xi\|_{H_{\rho}}+\left\|O_{t}\right\|_{H_{\rho}}+\frac{T^{1-\alpha-\rho}}{1-\alpha-\rho}\left(\sup _{v \in H_{\gamma}} \frac{\|F(v)\|_{H_{-\alpha}}}{1+\|v\|_{H}^{2}}\right)\left(1+\sup _{u \in[0, T]}\left\|Z_{u}\right\|_{H}^{2}\right)<\infty .
\end{aligned}
$$

This establishes items (ii) and (iii). Next note that (60) and Minkowski's integral inequality (see, e.g., [18, Proposition 8 in A.1]) ensure that for every $t \in(0, T]$ it holds that

$$
\int_{0}^{t}\left\|e^{(t-\kappa(u)) A} F\left(Z_{u}\right)\right\|_{\mathcal{L}^{p}\left(\mathbb{P} ; H_{\rho}\right)} d u \leq \frac{t^{1-\alpha-\rho}}{1-\alpha-\rho}\left(\sup _{v \in H_{\gamma}} \frac{1+\|F(v)\|_{H_{-\alpha}}}{1+\|v\|_{H}^{2}}\right)\left(1+\sup _{u \in[0, T]}\left\|Z_{u}\right\|_{\mathcal{L}^{2 p}(\mathbb{P} ; H)}^{2}\right) .
$$

The triangle inequality therefore establishes item (iii). This completes the proof of Lemma 3.2 .

Lemma 3.3. Assume Setting [3.1, let $p \in[1, \infty), \rho \in[0, \beta], \eta \in[\rho, \beta], \alpha_{1} \in[0,1-\rho), \alpha_{2} \in$ $[0,1-\eta)$, and assume for every $t \in[0, T]$ that $Z_{t}(\Omega) \subseteq H_{\rho}, \sup _{u \in[0, T]}\left\|Z_{u}\right\|_{H_{\rho}} \leq \sup _{u \in[0, T]}\left\|Y_{u}\right\|_{H_{\rho}}$, $\sup _{u \in[0, T]}\left\|Z_{u}\right\|_{\mathcal{L}^{2 p}\left(\mathbb{P} ; H_{\rho}\right)} \leq \sup _{u \in[0, T]}\left\|Y_{u}\right\|_{\mathcal{L}^{2 p}\left(\mathbb{P} ; H_{\rho}\right)}$, and

$$
\left(\sup _{v \in H_{\max \{\gamma, \rho\}}} \frac{\|F(v)\|_{H_{-\alpha_{2}}}}{1+\|v\|_{H_{\rho}}^{2}}\right)+\left(\sup _{v \in H_{\gamma}} \frac{\|F(v)\|_{H_{-\alpha}}}{1+\|v\|_{H}^{2}}\right)<\infty .
$$

Then

(i) it holds for every $t \in[0, T]$ that $Y_{t}(\Omega) \subseteq H_{\eta}$,

(ii) it holds for every $t \in[0, T]$ that

$$
\begin{aligned}
\left\|Y_{t}\right\|_{H_{\eta}} \leq & \|\xi\|_{H_{\eta}}+\left\|O_{t}\right\|_{H_{\eta}}+\frac{T^{1-\alpha_{2}-\eta}}{1-\alpha_{2}-\eta}\left(\sup _{v \in H_{\max \{\gamma, \rho\}}} \frac{\|F(v)\|_{H_{-\alpha}}}{1+\|v\|_{H_{\rho}}^{2}}\right) \\
\cdot & {\left[1+\|\xi\|_{H_{\rho}}+\sup _{u \in[0, T]}\left\|O_{u}\right\|_{H_{\rho}}\right.} \\
& \left.+\frac{T^{1-\alpha_{1}-\rho}}{1-\alpha_{1}-\rho}\left(\sup _{v \in H_{\gamma}} \frac{\|F(v)\|_{H_{-\alpha}}}{1+\|v\|_{H}^{2}}\right)\left(1+\sup _{u \in[0, T]}\left\|Z_{u}\right\|_{H}^{2}\right)\right]^{2}<\infty
\end{aligned}
$$

and

(iii) it holds for every $t \in[0, T]$ that

$$
\begin{aligned}
\left\|Y_{t}\right\|_{\mathcal{L}^{p}\left(\mathbb{P} ; H_{\eta}\right)} \leq & \|\xi\|_{\mathcal{L}^{p}\left(\mathbb{P} ; H_{\eta}\right)}+\left\|O_{t}\right\|_{\mathcal{L}^{p}\left(\mathbb{P} ; H_{\eta}\right)}+\frac{T^{1-\alpha_{2}-\eta}}{1-\alpha_{2}-\eta}\left(\sup _{v \in H_{\max \{\gamma, \rho\}}} \frac{1+\|F(v)\|_{H_{-\alpha_{2}}}}{1+\|v\|_{H_{\rho}}^{2}}\right) \\
\cdot & {\left[1+\|\xi\|_{\mathcal{L}^{2 p}\left(\mathbb{P} ; H_{\rho}\right)}+\sup _{u \in[0, T]}\left\|O_{u}\right\|_{\mathcal{L}^{2 p}\left(\mathbb{P} ; H_{\rho}\right)}\right.} \\
& \left.+\frac{T^{1-\alpha_{1}-\rho}}{1-\alpha_{1}-\rho}\left(\sup _{v \in H_{\gamma}} \frac{1+\|F(v)\|_{H_{-\alpha_{1}}}}{1+\|v\|_{H}^{2}}\right)\left(1+\sup _{u \in[0, T]}\left\|Z_{u}\right\|_{\mathcal{L}^{4 p}(\mathbb{P} ; H)}^{2}\right)\right]^{2} .
\end{aligned}
$$


Proof of Lemma 3.3. Throughout this proof assume w.l.o.g. that $\sup _{v \in H_{\gamma}}\|F(v)\|_{H}>0$. Note that the assumption that $\forall t \in[0, T]: \kappa(t) \leq t$ implies that for every $t \in(0, T]$ it holds that

$$
\begin{aligned}
& \int_{0}^{t}\left\|e^{(t-\kappa(u)) A} F\left(Z_{u}\right)\right\|_{H_{\eta}} d u \leq \int_{0}^{t}\left\|(-A)^{\alpha_{2}+\eta} e^{(t-\kappa(u)) A}\right\|_{L(H)}\left\|F\left(Z_{u}\right)\right\|_{H_{-\alpha_{2}}} d u \\
& \leq \int_{0}^{t}(t-\kappa(u))^{-\alpha_{2}-\eta}\left\|F\left(Z_{u}\right)\right\|_{H_{-\alpha_{2}}} d u \\
& \leq\left(\sup _{v \in H_{\max \{\gamma, \rho\}}} \frac{\|F(v)\|_{H_{-\alpha}}}{1+\|v\|_{H_{\rho}}^{2}}\right) \int_{0}^{t}(t-\kappa(u))^{-\alpha_{2}-\eta}\left(1+\left\|Z_{u}\right\|_{H_{\rho}}^{2}\right) d u \\
& \leq\left(\sup _{v \in H_{\max \{\gamma, \rho\}}} \frac{\|F(v)\|_{H_{-}}}{1+\|v\|_{H_{\rho}}^{2}}\right) \int_{0}^{t}(t-u)^{-\alpha_{2}-\eta}\left(1+\left\|Z_{u}\right\|_{H_{\rho}}^{2}\right) d u
\end{aligned}
$$

Hence, we obtain that for every $t \in(0, T]$ it holds that

$$
\begin{aligned}
\int_{0}^{t}\left\|e^{(t-\kappa(u)) A} F\left(Z_{u}\right)\right\|_{H_{\eta}} d u & \leq\left(\sup _{v \in H_{\max \{\gamma, \rho\}}} \frac{\|F(v)\|_{H_{-\alpha}}}{1+\|v\|_{H_{\rho}}^{2}}\right) \frac{t^{1-\alpha_{2}-\eta}}{1-\alpha_{2}-\eta}\left(1+\sup _{u \in[0, T]}\left\|Z_{u}\right\|_{H_{\rho}}^{2}\right) \\
& \leq\left(\sup _{v \in H_{\max \{\gamma, \rho\}}} \frac{\|F(v)\|_{H_{-\alpha}}}{1+\|v\|_{H_{\rho}}^{2}}\right) \frac{t^{1-\alpha_{2}-\eta}}{1-\alpha_{2}-\eta}\left(1+\sup _{u \in[0, T]}\left\|Z_{u}\right\|_{H_{\rho}}\right)^{2} .
\end{aligned}
$$

Next observe that (67) and Minkowski's integral inequality (see, e.g., [18, Proposition 8 in A.1]) ensure that for every $t \in(0, T]$ it holds that

$$
\begin{aligned}
& \int_{0}^{t}\left\|e^{(t-\kappa(u)) A} F\left(Z_{u}\right)\right\|_{\mathcal{L}^{p}\left(\mathbb{P} ; H_{\eta}\right)} d u \\
& \leq\left(\sup _{v \in H_{\max \{\gamma, \rho\}}} \frac{1+\|F(v)\|_{H_{-\alpha}}}{1+\|v\|_{H_{\rho}}^{2}}\right) \frac{t^{1-\alpha_{2}-\eta}}{1-\alpha_{2}-\eta}\left(1+\sup _{u \in[0, T]}\left\|Z_{u}\right\|_{\mathcal{L}^{2 p}\left(\mathbb{P} ; H_{\rho}\right)}^{2}\right) \\
& \leq\left(\sup _{v \in H_{\max \{\gamma, \rho\}}} \frac{1+\|F(v)\|_{H_{-\alpha}}}{1+\|v\|_{H_{\rho}}^{2}}\right) \frac{t^{1-\alpha_{2}-\eta}}{1-\alpha_{2}-\eta}\left(1+\sup _{u \in[0, T]}\left\|Z_{u}\right\|_{\mathcal{L}^{2 p}\left(\mathbb{P} ; H_{\rho}\right)}\right)^{2} .
\end{aligned}
$$

Moreover, note that (64) and Lemma 3.2 (with $p=2 p, \rho=\rho, \alpha=\alpha_{1}$ in the notation of Lemma 3.2) imply that

(a) it holds for every $t \in[0, T]$ that $Y_{t}(\Omega) \subseteq H_{\rho}$,

(b) it holds that

$$
\begin{aligned}
& \sup _{u \in[0, T]}\left\|Z_{u}\right\|_{H_{\rho}} \leq \sup _{u \in[0, T]}\left\|Y_{u}\right\|_{H_{\rho}} \\
& \leq\|\xi\|_{H_{\rho}}+\sup _{u \in[0, T]}\left\|O_{u}\right\|_{H_{\rho}}+\frac{T^{1-\alpha_{1}-\rho}}{1-\alpha_{1}-\rho}\left(\sup _{v \in H_{\gamma}} \frac{\|F(v)\|_{H_{-\alpha}}}{1+\|v\|_{H}^{2}}\right)\left(1+\sup _{u \in[0, T]}\left\|Z_{u}\right\|_{H}^{2}\right)<\infty
\end{aligned}
$$

and

(c) it holds that

$$
\begin{aligned}
\sup _{u \in[0, T]}\left\|Z_{u}\right\|_{\mathcal{L}^{2 p}\left(\mathbb{P} ; H_{\rho}\right)} \leq & \sup _{u \in[0, T]}\left\|Y_{u}\right\|_{\mathcal{L}^{2 p}\left(\mathbb{P} ; H_{\rho}\right)} \leq\|\xi\|_{\mathcal{L}^{2 p}\left(\mathbb{P} ; H_{\rho}\right)}+\sup _{u \in[0, T]}\left\|O_{u}\right\|_{\mathcal{L}^{2 p}\left(\mathbb{P} ; H_{\rho}\right)} \\
& +\frac{T^{1-\alpha_{1}-\rho}}{1-\alpha_{1}-\rho}\left(\sup _{v \in H_{\gamma}} \frac{1+\|F(v)\|_{H_{-\alpha}}}{1+\|v\|_{H}^{2}}\right)\left(1+\sup _{u \in[0, T]}\left\|Z_{u}\right\|_{\mathcal{L}^{4 p}(\mathbb{P} ; H)}^{2}\right)
\end{aligned}
$$


Observe that the triangle inequality, (64), (68) and item (bholds that $Y_{t}(\Omega) \subseteq H_{\eta}$ and

$$
\begin{aligned}
\left\|Y_{t}\right\|_{H_{\eta}} \leq & \|\xi\|_{H_{\eta}}+\int_{0}^{t}\left\|e^{(t-\kappa(u)) A} F\left(Z_{u}\right)\right\|_{H_{\eta}} d u+\left\|O_{t}\right\|_{H_{\eta}} \\
\leq & \|\xi\|_{H_{\eta}}+\left\|O_{t}\right\|_{H_{\eta}}+\left(\sup _{v \in H_{\max \{\gamma, \rho\}}} \frac{\|F(v)\|_{H_{-}-\alpha_{2}}}{1+\|v\|_{H_{\rho}}^{2}}\right) \frac{T^{1-\alpha_{2}-\eta}}{1-\alpha_{2}-\eta}\left(1+\sup _{u \in[0, T]}\left\|Z_{u}\right\|_{H_{\rho}}^{2}\right) \\
\leq & \|\xi\|_{H_{\eta}}+\left\|O_{t}\right\|_{H_{\eta}}+\frac{T^{1-\alpha_{2}-\eta}}{1-\alpha_{2}-\eta}\left(\sup _{v \in H_{\max \{\gamma, \rho\}}} \frac{\|F(v)\|_{H_{-\alpha}}}{1+\|v\|_{H_{\rho}}^{2}}\right) \\
& \cdot\left[1+\|\xi\|_{H_{\rho}}+\sup _{u \in[0, T]}\left\|O_{u}\right\|_{H_{\rho}}\right. \\
& \left.\quad+\frac{T^{1-\alpha_{1}-\rho}}{1-\alpha_{1}-\rho}\left(\sup _{v \in H_{\gamma}} \frac{\|F(v)\|_{H_{-\alpha}}}{1+\|v\|_{H}^{2}}\right)\left(1+\sup _{u \in[0, T]}\left\|Z_{u}\right\|_{H}^{2}\right)\right]^{2}<\infty .
\end{aligned}
$$

This establishes items (ii) and (ii). Furthermore, observe that the triangle inequality and (69) prove that for every $t \in[0, T]$ it holds that

$$
\begin{aligned}
\left\|Y_{t}\right\|_{\mathcal{L}^{p}\left(\mathbb{P} ; H_{\eta}\right)} \leq & \|\xi\|_{\mathcal{L}^{p}\left(\mathbb{P} ; H_{\eta}\right)}+\int_{0}^{t}\left\|e^{(t-\kappa(u)) A} F\left(Z_{u}\right)\right\|_{\mathcal{L}^{p}\left(\mathbb{P} ; H_{\eta}\right)} d u+\left\|O_{t}\right\|_{\mathcal{L}^{p}\left(\mathbb{P} ; H_{\eta}\right)} \\
\leq & \|\xi\|_{\mathcal{L}^{p}\left(\mathbb{P} ; H_{\eta}\right)}+\left\|O_{t}\right\|_{\mathcal{L}^{p}\left(\mathbb{P} ; H_{\eta}\right)} \\
& +\left(\sup _{v \in H_{\max \{\gamma, \rho\}}} \frac{1+\|F(v)\|_{H_{-\alpha}}}{1+\|v\|_{H_{\rho}}^{2}}\right) \frac{T^{1-\alpha_{2}-\eta}}{1-\alpha_{2}-\eta}\left(1+\sup _{u \in[0, T]}\left\|Z_{u}\right\|_{\mathcal{L}^{2 p}\left(\mathbb{P} ; H_{\rho}\right)}\right)^{2}
\end{aligned}
$$

Combining this and item (ㄷ) establishes item (iii). The proof of Lemma 3.3 is thus completed.

Lemma 3.4. Assume Setting [3.1, let $p \in[1, \infty), \rho \in[0, \beta], \eta \in[\rho, \beta], \iota \in$ $[\eta, \beta], \quad \alpha_{1} \in[0,1-\rho), \quad \alpha_{2} \in[0,1-\eta)$, and assume for every $t \in[0, T]$ that $Z_{t}(\Omega) \subseteq H_{\eta}, \sup _{u \in[0, T]}\left\|Z_{u}\right\|_{H_{\rho}} \leq \sup _{u \in[0, T]}\left\|Y_{u}\right\|_{H_{\rho}}, \sup _{u \in[0, T]}\left\|Z_{u}\right\|_{H_{\eta}} \leq \sup _{u \in[0, T]}\left\|Y_{u}\right\|_{H_{\eta}}$, $\sup _{u \in[0, T]}\left\|Z_{u}\right\|_{\mathcal{L}^{4 p}\left(\mathbb{P} ; H_{\rho}\right)} \leq \sup _{u \in[0, T]}\left\|Y_{u}\right\|_{\mathcal{L}^{4 p}\left(\mathbb{P} ; H_{\rho}\right)}, \sup _{u \in[0, T]}\left\|Z_{u}\right\|_{\mathcal{L}^{2 p}\left(\mathbb{P} ; H_{\eta}\right)} \leq \sup _{u \in[0, T]}\left\|Y_{u}\right\|_{\mathcal{L}^{2 p}\left(\mathbb{P} ; H_{\eta}\right)}$, and

$$
\left[\sup _{v \in H_{\max \{\gamma, \eta\}}} \frac{\|F(v)\|_{H}}{1+\|v\|_{H_{\eta}}^{2}}\right]+\left[\sup _{v \in H_{\max \{\gamma, \rho\}}} \frac{\|F(v)\|_{H_{-\alpha}}}{1+\|v\|_{H_{\rho}}^{2}}\right]+\left[\sup _{v \in H_{\gamma}} \frac{\|F(v)\|_{H_{-} \alpha_{1}}}{1+\|v\|_{H}^{2}}\right]<\infty
$$

Then

(i) it holds for every $t \in[0, T]$ that $Y_{t}(\Omega) \subseteq H_{\iota}$,

(ii) it holds for every $t \in[0, T]$ that

$$
\begin{aligned}
& \left\|Y_{t}\right\|_{H_{\iota}} \leq\|\xi\|_{H_{\iota}}+\left\|O_{t}\right\|_{H_{\iota}}+\frac{T^{1-\iota}}{1-\iota}\left(\sup _{v \in H_{\max \{\gamma, \eta\}}} \frac{\|F(v)\|_{H}}{1+\|v\|_{H_{\eta}}^{2}}\right) \\
& \cdot\left[1+\|\xi\|_{H_{\eta}}+\sup _{u \in[0, T]}\left\|O_{u}\right\|_{H_{\eta}}+\frac{T^{1-\alpha_{2}-\eta}}{1-\alpha_{2}-\eta}\left(\sup _{v \in H_{\max \{\gamma, \rho\}}} \frac{\|F(v)\|_{H_{-}}}{1+\|v\|_{H_{\rho}}^{2}}\right)\right. \\
& \cdot\left[1+\|\xi\|_{H_{\rho}}+\sup _{u \in[0, T]}\left\|O_{u}\right\|_{H_{\rho}}\right. \\
& \left.\left.\quad+\frac{T^{1-\alpha_{1}-\rho}}{1-\alpha_{1}-\rho}\left(\sup _{v \in H_{\gamma}} \frac{\|F(v)\|_{H_{-\alpha}}}{1+\|v\|_{H}^{2}}\right)\left(1+\sup _{u \in[0, T]}\left\|Z_{u}\right\|_{H}^{2}\right)\right]^{2}\right]^{2}<\infty,
\end{aligned}
$$

and 
(iii) it holds for every $t \in[0, T]$ that

$$
\begin{aligned}
& \left\|Y_{t}\right\|_{\mathcal{L}^{p}\left(\mathbb{P} ; H_{\iota}\right)} \leq\|\xi\|_{\mathcal{L}^{p}\left(\mathbb{P} ; H_{\iota}\right)}+\left\|O_{t}\right\|_{\mathcal{L}^{p}\left(\mathbb{P} ; H_{\iota}\right)}+\frac{T^{1-\iota}}{1-\iota}\left(\sup _{v \in H_{\max \{\gamma, \eta\}}} \frac{1+\|F(v)\|_{H}}{1+\|v\|_{H \eta}^{2}}\right) \\
& \cdot\left[1+\|\xi\|_{\mathcal{L}^{2 p}\left(\mathbb{P} ; H_{\eta}\right)}+\sup _{u \in[0, T]}\left\|O_{u}\right\|_{\mathcal{L}^{2 p}\left(\mathbb{P} ; H_{\eta}\right)}+\frac{T^{1-\alpha_{2}-\eta}}{1-\alpha_{2}-\eta}\left(\sup _{v \in H_{\max \{\gamma, \rho\}}} \frac{1+\|F(v)\|_{H_{-\alpha_{2}}}}{1+\|v\|_{H_{\rho}}^{2}}\right)\right. \\
& \cdot\left[1+\|\xi\|_{\mathcal{L}^{4 p}\left(\mathbb{P} ; H_{\rho}\right)}+\sup _{u \in[0, T]}\left\|O_{u}\right\|_{\mathcal{L}^{4 p}\left(\mathbb{P} ; H_{\rho}\right)}\right. \\
& \left.\left.\quad+\frac{T^{1-\alpha_{1}-\rho}}{1-\alpha_{1}-\rho}\left(\sup _{v \in H_{\gamma}} \frac{1+\|F(v)\|_{H_{-\alpha}}}{1+\|v\|_{H}^{2}}\right)\left(1+\sup _{u \in[0, T]}\left\|Z_{u}\right\|_{\mathcal{L}^{8 p}(\mathbb{P} ; H)}^{2}\right)\right]^{2}\right]^{2} \cdot
\end{aligned}
$$

Proof of Lemma 3.4. Throughout this proof assume w.l.o.g. that $\sup _{v \in H_{\gamma}}\|F(v)\|_{H}>0$. Observe that the assumption that $\forall t \in[0, T]: \kappa(t) \leq t$ implies that for every $t \in(0, T]$ it holds that

$$
\begin{aligned}
& \int_{0}^{t}\left\|e^{(t-\kappa(u)) A} F\left(Z_{u}\right)\right\|_{H_{\iota}} d u \leq \int_{0}^{t}(t-\kappa(u))^{-\iota}\left\|F\left(Z_{u}\right)\right\|_{H} d u \\
& \leq\left(\sup _{v \in H_{\max \{\gamma, \eta\}}} \frac{\|F(v)\|_{H}}{1+\|v\|_{H_{\eta}}^{2}}\right) \int_{0}^{t}(t-\kappa(u))^{-\iota}\left(1+\left\|Z_{u}\right\|_{H_{\eta}}^{2}\right) d u \\
& \leq\left(\sup _{v \in H_{\max \{\gamma, \eta\}}} \frac{\|F(v)\|_{H}}{1+\|v\|_{H_{\eta}}^{2}}\right) \int_{0}^{t}(t-u)^{-\iota}\left(1+\left\|Z_{u}\right\|_{H_{\eta}}^{2}\right) d u .
\end{aligned}
$$

Hence, we obtain that for every $t \in(0, T]$ it holds that

$$
\begin{aligned}
\int_{0}^{t}\left\|e^{(t-\kappa(u)) A} F\left(Z_{u}\right)\right\|_{H_{\iota}} d u & \leq\left(\sup _{v \in H_{\max \{\gamma, \eta\}}} \frac{\|F(v)\|_{H}}{1+\|v\|_{H_{\eta}}^{2}}\right) \frac{t^{1-\iota}}{1-\iota}\left(1+\sup _{u \in[0, T]}\left\|Z_{u}\right\|_{H_{\eta}}^{2}\right) \\
& \leq\left(\sup _{v \in H_{\max \{\gamma, \eta\}}} \frac{\|F(v)\|_{H}}{1+\|v\|_{H_{\eta}}^{2}}\right) \frac{t^{1-\iota}}{1-\iota}\left(1+\sup _{u \in[0, T]}\left\|Z_{u}\right\|_{H_{\eta}}\right)^{2} .
\end{aligned}
$$

Moreover, note that (77) and Minkowski's integral inequality (see, e.g., [18, Proposition 8 in A.1]) prove that for every $t \in(0, T]$ it holds that

$$
\begin{aligned}
& \int_{0}^{t}\left\|e^{(t-\kappa(u)) A} F\left(Z_{u}\right)\right\|_{\mathcal{L}^{p}\left(\mathbb{P} ; H_{\iota}\right)} d u \\
& \leq\left(\sup _{v \in H_{\max \{\gamma, \eta\}}} \frac{\|F(v)\|_{H}}{1+\|v\|_{H_{\eta}}^{2}}\right) \frac{t^{1-\iota}}{1-\iota}\left(1+\sup _{u \in[0, T]}\left\|Z_{u}\right\|_{\mathcal{L}^{2 p}\left(\mathbb{P} ; H_{\eta}\right)}^{2}\right) \\
& \leq\left(\sup _{v \in H_{\max \{\gamma, \eta\}}} \frac{\|F(v)\|_{H}}{1+\|v\|_{H_{\eta}}^{2}}\right) \frac{t^{1-\iota}}{1-\iota}\left(1+\sup _{u \in[0, T]}\left\|Z_{u}\right\|_{\mathcal{L}^{2 p}\left(\mathbb{P} ; H_{\eta}\right)}\right)^{2} .
\end{aligned}
$$

Next observe that (74), the assumption that $\sup _{u \in[0, T]}\left\|Z_{u}\right\|_{H_{\rho}} \leq \sup _{u \in[0, T]}\left\|Y_{u}\right\|_{H_{\rho}}$, the assumption that $\sup _{u \in[0, T]}\left\|Z_{u}\right\|_{\mathcal{L}^{4 p}\left(\mathbb{P} ; H_{\rho}\right)} \leq \sup _{u \in[0, T]}\left\|Y_{u}\right\|_{\mathcal{L}^{4 p}\left(\mathbb{P} ; H_{\rho}\right)}$, and Lemma 3.3 (with $p=2 p, \rho=\rho, \eta=\eta$, $\alpha_{1}=\alpha_{1}, \alpha_{2}=\alpha_{2}$ in the notation of Lemma 3.3) show that

(a) it holds for every $t \in[0, T]$ that $Y_{t}(\Omega) \subseteq H_{\eta}$,

(b) it holds that

$$
\begin{aligned}
& \sup _{u \in[0, T]}\left\|Z_{u}\right\|_{H_{\eta}} \leq \sup _{u \in[0, T]}\left\|Y_{u}\right\|_{H_{\eta}} \\
& \leq\|\xi\|_{H_{\eta}}+\sup _{u \in[0, T]}\left\|O_{u}\right\|_{H_{\eta}}+\frac{T^{1-\alpha_{2}-\eta}}{1-\alpha_{2}-\eta}\left(\sup _{v \in H_{\max \{\gamma, \rho\}}} \frac{\|F(v)\|_{H_{-\alpha}}}{1+\|v\|_{H_{\rho}}^{2}}\right) \\
& \cdot\left[1+\|\xi\|_{H_{\rho}}+\sup _{u \in[0, T]}\left\|O_{u}\right\|_{H_{\rho}}\right. \\
& \left.\quad+\frac{T^{1-\alpha_{1}-\rho}}{1-\alpha_{1}-\rho}\left(\sup _{v \in H_{\gamma}} \frac{\|F(v)\|_{H_{-\alpha}}}{1+\|v\|_{H}^{2}}\right)\left(1+\sup _{u \in[0, T]}\left\|Z_{u}\right\|_{H}^{2}\right)\right]^{2}<\infty,
\end{aligned}
$$


and

(c) it holds that

$$
\begin{aligned}
& \sup _{u \in[0, T]}\left\|Z_{u}\right\|_{\mathcal{L}^{2 p}\left(\mathbb{P} ; H_{\eta}\right)} \leq \sup _{u \in[0, T]}\left\|Y_{u}\right\|_{\mathcal{L}^{2 p}\left(\mathbb{P} ; H_{\eta}\right)} \\
& \leq\|\xi\|_{\mathcal{L}^{2 p}\left(\mathbb{P} ; H_{\eta}\right)}+\sup _{u \in[0, T]}\left\|O_{u}\right\|_{\mathcal{L}^{2 p}\left(\mathbb{P} ; H_{\eta}\right)}+\frac{T^{1-\alpha_{2}-\eta}}{1-\alpha_{2}-\eta}\left(\sup _{v \in H_{\max \{\gamma, \rho\}}} \frac{1+\|F(v)\|_{H_{-\alpha_{2}}}}{1+\|v\|_{H_{\rho}}^{2}}\right) \\
& \cdot\left[1+\|\xi\|_{\mathcal{L}^{4 p}\left(\mathbb{P} ; H_{\rho}\right)}+\sup _{u \in[0, T]}\left\|O_{u}\right\|_{\mathcal{L}^{4 p}\left(\mathbb{P} ; H_{\rho}\right)}\right. \\
& \left.\quad+\frac{T^{1-\alpha_{1}-\rho}}{1-\alpha_{1}-\rho}\left(\sup _{v \in H_{\gamma}} \frac{1+\|F(v)\|_{H_{-\alpha_{1}}}}{1+\|v\|_{H}^{2}}\right)\left(1+\sup _{u \in[0, T]}\left\|Z_{u}\right\|_{\mathcal{L}^{8 p}(\mathbb{P} ; H)}^{2}\right)\right]^{2} \cdot
\end{aligned}
$$

Note that the triangle inequality, (174), (78), and item (b) ensure that for every $t \in[0, T]$ it holds that $Y_{t}(\Omega) \subseteq H_{\iota}$ and

$$
\begin{aligned}
\left\|Y_{t}\right\|_{H_{\iota} \leq} \leq & \|\xi\|_{H_{\iota}}+\int_{0}^{t}\left\|e^{(t-\kappa(u)) A} F\left(Z_{u}\right)\right\|_{H_{\iota}} d u+\left\|O_{t}\right\|_{H_{\iota}} \\
\leq & \|\xi\|_{H_{\iota}}+\left\|O_{t}\right\|_{H_{\iota}}+\left(\sup _{v \in H_{\max \{\gamma, \eta\}}} \frac{\|F(v)\|_{H}}{1+\|v\|_{H_{\eta}}^{2}}\right) \frac{T^{1-\iota}}{1-\iota}\left(1+\sup _{u \in[0, T]}\left\|Z_{u}\right\|_{H_{\eta}}\right)^{2} \\
\leq & \|\xi\|_{H_{\iota}}+\left\|O_{t}\right\|_{H_{\iota}}+\frac{T^{1-\iota}}{1-\iota}\left(\sup _{v \in H_{\max \{\gamma, \eta\}}} \frac{\|F(v)\|_{H}}{1+\|v\|_{H_{\eta}}^{2}}\right) \\
\cdot & {\left[1+\|\xi\|_{H_{\eta}}+\sup _{u \in[0, T]}\left\|O_{u}\right\|_{H_{\eta}}+\frac{T^{1-\alpha_{2}-\eta}}{1-\alpha_{2}-\eta}\left(\sup _{v \in H_{\max \{\gamma, \rho\}}} \frac{\|F(v)\|_{H_{-\alpha}}}{1+\|v\|_{H_{\rho}}^{2}}\right)\right.} \\
\cdot & {\left[1+\|\xi\|_{H_{\rho}}+\sup _{u \in[0, T]}\left\|O_{u}\right\|_{H_{\rho}}\right.} \\
& \left.\left.+\frac{T^{1-\alpha_{1}-\rho}}{1-\alpha_{1}-\rho}\left(\sup _{v \in H_{\gamma}} \frac{\|F(v)\|_{H_{-} \alpha_{1}}}{1+\|v\|_{H}^{2}}\right)\left(1+\sup _{u \in[0, T]}\left\|Z_{u}\right\|_{H}^{2}\right)\right]^{2}\right]^{2}<\infty .
\end{aligned}
$$

This establishes items (ii) and (iii). Furthermore, observe that the triangle inequality and (79) prove that for every $t \in[0, T]$ it holds that

$$
\begin{aligned}
\left\|Y_{t}\right\|_{\mathcal{L}^{p}\left(\mathbb{P} ; H_{\iota}\right)} \leq & \|\xi\|_{\mathcal{L}^{p}\left(\mathbb{P} ; H_{\iota}\right)}+\int_{0}^{t}\left\|e^{(t-\kappa(u)) A} F\left(Z_{u}\right)\right\|_{\mathcal{L}^{p}\left(\mathbb{P} ; H_{\iota}\right)} d u+\left\|O_{t}\right\|_{\mathcal{L}^{p}\left(\mathbb{P} ; H_{\iota}\right)} \\
\leq & \|\xi\|_{\mathcal{L}^{p}\left(\mathbb{P} ; H_{\iota}\right)}+\left\|O_{t}\right\|_{\mathcal{L}^{p}\left(\mathbb{P} ; H_{\iota}\right)} \\
& +\left(\sup _{v \in H_{\max \{\gamma, \eta\}}} \frac{1+\|F(v)\|_{H}}{1+\|v\|_{H_{\eta}}^{2}}\right) \frac{T^{1-\iota}}{1-\iota}\left(1+\sup _{u \in[0, T]}\left\|Z_{u}\right\|_{\mathcal{L}^{2 p}\left(\mathbb{P} ; H_{\eta}\right)}\right)^{2} .
\end{aligned}
$$

Combining this and item (C) establishes item (iii). The proof of Lemma 3.4 is thus completed.

\section{Properties of the nonlinearity}

In this section we recall and derive in Subsection 4.1 and in Subsection 4.2 some partially wellknown properties of certain Sobolev spaces and the nonlinearity appearing in the stochastic Burgers equations, respectively. We employ these results to establish in Theorem 5.10 in Section 5 below the main result of this article.

Setting 4.1. Assume Setting 1.2, let $\lambda: \mathcal{B}((0,1)) \rightarrow[0,1]$ be the Lebesgue-Borel measure on $(0,1)$, for every measure space $(\Omega, \mathcal{F}, \mu)$, every measurable space $(S, \mathcal{S})$, every set $R$, and every 
function $f: \Omega \rightarrow R$ let $[f]_{\mu, \mathcal{S}}=\{g \in \mathcal{M}(\mathcal{F}, \mathcal{S}):(\exists D \in \mathcal{F}: \mu(D)=0$ and $\{\omega \in \Omega: f(\omega) \neq$ $g(\omega)\} \subseteq D)\}$, let $c_{0} \in(0, \infty), c_{1} \in \mathbb{R}$, assume that $\left(H,\langle\cdot, \cdot\rangle_{H},\|\cdot\|_{H}\right)=\left(L^{2}(\lambda ; \mathbb{R}),\langle\cdot, \cdot\rangle_{L^{2}(\lambda ; \mathbb{R})}\right.$, $\left.\|\cdot\|_{L^{2}(\lambda ; \mathbb{R})}\right)$, let $\left(e_{n}\right)_{n \in \mathbb{N}} \subseteq H$ satisfy for every $n \in \mathbb{N}$ that $e_{n}=\left[(\sqrt{2} \sin (n \pi x))_{x \in(0,1)}\right]_{\lambda, \mathcal{B}(\mathbb{R})}$, assume that $\mathbb{H}=\left\{e_{n}: n \in \mathbb{N}\right\}$, assume for every $n \in \mathbb{N}$ that $\mathfrak{v}_{e_{n}}=-c_{0} \pi^{2} n^{2}$, for every $v \in W^{1,2}((0,1), \mathbb{R})$ let $\partial v \in H$ satisfy for every $\varphi \in \mathcal{C}_{c p t}^{\infty}((0,1), \mathbb{R})$ that $\left\langle\partial v,[\varphi]_{\lambda, \mathcal{B}(\mathbb{R})}\right\rangle_{H}=-\left\langle v,\left[\varphi^{\prime}\right]_{\lambda, \mathcal{B}(\mathbb{R})}\right\rangle_{H}$, and let $F: H_{1 / 2} \rightarrow H$ be the function which satisfies for every $w \in H_{1 / 2}$ that $F(w)=c_{1} w \partial w$.

Note that for every $s \in[0, \infty), p \in[1, \infty)$ it holds that $\left(W^{s, p}((0,1), \mathbb{R}),\|\cdot\|_{W^{s, p}((0,1), \mathbb{R})}\right)$ is the Sobolev-Slobodeckij space with smoothness parameter $s$ and integrability parameter $p$ of equivalence classes of $\mathcal{B}((0,1)) / \mathcal{B}(\mathbb{R})$-measurable functions.

\subsection{Auxiliary results on Sobolev and interpolation spaces}

In this subsection we recall some elementary properties of the involved Sobolev and interpolation spaces. Lemmas 4.2 4.5, Lemma 4.6 (cf., e.g., Fujiwara [13]), Lemmas 4.74.10, Lemma 4.11 (cf., e.g., Brezis [5, Exercise 8.15 and (42) in the section Comments on Chapter 8] and Nirenberg [32]), and Lemma4.12 (see, e.g., Sell \& You [37, Theorem B.2]) below are used for the regularity analysis of the considered nonlinearity in Subsection 4.2 below.

Lemma 4.2. Assume Setting 4.1. Then it holds for every $\rho \in[1 / 2, \infty)$ that $\sum_{h \in \mathbb{H}}\left|\mathfrak{v}_{h}\right|^{-2 \rho} \leq\left|c_{0}\right|^{-2 \rho} / 6$, $\sup _{h \in \mathbb{H}}\|\partial h\|_{H}\left|\mathfrak{v}_{h}\right|^{-\rho} \leq\left|c_{0}\right|^{-\rho}$, and $\sup _{h \in \mathbb{H}}\|h\|_{L^{\infty}(\lambda ; \mathbb{R})}=\sqrt{2}$.

Proof of Lemma 4.2. First, observe that

$$
\begin{aligned}
\sum_{h \in \mathbb{H}}\left|\mathfrak{v}_{h}\right|^{-2 \rho} & =\sum_{n \in \mathbb{N}}\left|c_{0} \pi^{2} n^{2}\right|^{-2 \rho}=\left|c_{0}\right|^{-2 \rho} \pi^{-4 \rho} \sum_{n \in \mathbb{N}} n^{-4 \rho} \\
& \leq\left|c_{0}\right|^{-2 \rho} \pi^{-2} \sum_{n \in \mathbb{N}} n^{-2}=\left|c_{0}\right|^{-2 \rho} \pi^{-2} \frac{\pi^{2}}{6}
\end{aligned}
$$

Moreover, note that for every $n \in \mathbb{N}$ it holds that

$$
\begin{aligned}
\left\|\partial e_{n}\right\|_{H}\left|\mathfrak{v}_{e_{n}}\right|^{-\rho} & =\left\|\left[(\pi n \sqrt{2} \cos (n \pi x))_{x \in(0,1)}\right]_{\lambda, \mathcal{B}(\mathbb{R})}\right\|_{H}\left|c_{0} \pi^{2} n^{2}\right|^{-\rho} \\
& =\pi n\left|c_{0} \pi^{2} n^{2}\right|^{-\rho}=\frac{\left|c_{0}\right|^{-\rho}}{(\pi n)^{2 \rho-1}} \leq\left|c_{0}\right|^{-\rho}
\end{aligned}
$$

In addition, observe that for every $n \in \mathbb{N}, x \in(0,1)$ it holds that

$$
|\sqrt{2} \sin (\pi n x)| \leq \sqrt{2} .
$$

This completes the proof of Lemma 4.2.

Lemma 4.3. Assume Setting 4.1. Then

(i) it holds that $W_{0}^{1,2}((0,1), \mathbb{R}) \subseteq H_{1 / 2}$ continuously,

(ii) it holds that $H_{1 / 2} \subseteq W_{0}^{1,2}((0,1), \mathbb{R})$ continuously,

(iii) it holds that $W_{0}^{1,2}((0,1), \mathbb{R}) \subseteq L^{\infty}(\lambda ; \mathbb{R})$ continuously,

(iv) it holds for every $v \in H_{1 / 2}$ that $\|\partial v\|_{H}=\left|c_{0}\right|^{-1 / 2}\|v\|_{H_{1 / 2}}$, and

(v) it holds for every $v \in H_{1 / 2}$ that $\|v\|_{L^{\infty}(\lambda ; \mathbb{R})} \leq\left|3 c_{0}\right|^{-1 / 2}\|v\|_{H_{1 / 2}}$. 
Proof of Lemma 4.3. Note that, e.g., Lunardi [30, Example 4.34] ensures that

$$
H_{1 / 2}=W_{0}^{1,2}((0,1), \mathbb{R}) .
$$

This, the fact that for every $v \in W^{1,2}((0,1), \mathbb{R})$ it holds that

$$
\|v\|_{W^{1,2}((0,1), \mathbb{R})}^{2}=\|v\|_{H}^{2}+\|\partial v\|_{H}^{2},
$$

and the fact that for every $v \in H_{1 / 2}$ it holds that

$$
\|v\|_{H_{1 / 2}}=\sqrt{c_{0}}\|\partial v\|_{H}
$$

(see, e.g., [23, Lemma 6.1]) establish item (ii). Moreover, observe that (87)-(89) and Poincaré's inequality (see, e.g., Brezis [5, Proposition 8.13]) show item (iii). Next note that Lemma 4.2 (with $\rho=1 / 2$ in the notation of Lemma 4.2) and, e.g., [20, Lemma 4.3] (with $d=1, \mathbb{H}=\mathbb{H}, \rho=1 / 2$, $v=v$ for $v \in H_{1 / 2}$ in the notation of [20, Lemma 4.3]) prove that for every $v \in H_{1 / 2}$ it holds that

$$
\|v\|_{L^{\infty}(\lambda ; \mathbb{R})} \leq\|v\|_{H_{1 / 2}}\left(\sup _{h \in \mathbb{H}}\|h\|_{L^{\infty}(\lambda ; \mathbb{R})}\right)\left[\sum_{h \in \mathbb{H}}\left|\mathfrak{v}_{h}\right|^{-1}\right]^{1 / 2} \leq\left|3 c_{0}\right|^{-1 / 2}\|v\|_{H_{1 / 2}} .
$$

This and item (ii) establish item (iii). Moreover, note that (89) shows item (iv). In addition, observe that (90) establishes item ( $(\mathbf{v})$. The proof of Lemma 4.3 is thus completed.

Lemma 4.4. Assume Setting 4.1 and let $u \in W_{0}^{1,2}((0,1), \mathbb{R}), v \in W^{1,2}((0,1), \mathbb{R})$. Then it holds that

$$
\langle\partial u, v\rangle_{H}=-\langle u, \partial v\rangle_{H}
$$

Proof of Lemma 4.4. Throughout this proof let $\left(\mathbf{u}_{n}\right)_{n \in \mathbb{N}} \subseteq \mathcal{C}_{c}^{\infty}(\mathbb{R}, \mathbb{R}),\left(\mathbf{v}_{n}\right)_{n \in \mathbb{N}} \subseteq \mathcal{C}_{c}^{\infty}(\mathbb{R}, \mathbb{R})$, $\left(u_{n}\right)_{n \in \mathbb{N}} \subseteq W_{0}^{1,2}((0,1), \mathbb{R}),\left(v_{n}\right)_{n \in \mathbb{N}} \subseteq W^{1,2}((0,1), \mathbb{R})$ satisfy for every $n \in \mathbb{N}, x \in((-\infty, 0] \cup[1, \infty))$ that $\mathbf{u}_{n}(x)=0, u_{n}=\left[\left.\mathbf{u}_{n}\right|_{(0,1)}\right]_{\lambda, \mathcal{B}(\mathbb{R})}, v_{n}=\left[\left.\mathbf{v}_{n}\right|_{(0,1)}\right]_{\lambda, \mathcal{B}(\mathbb{R})}$, and $\lim \sup _{m \rightarrow \infty}\left(\left\|u-u_{m}\right\|_{W^{1,2}((0,1), \mathbb{R})}+\right.$ $\left.\left\|v-v_{m}\right\|_{W^{1,2}((0,1), \mathbb{R})}\right)=0$. Observe that integration by parts and the fact that for every $n \in \mathbb{N}$ it holds that $\mathbf{u}_{n}(0)=\mathbf{u}_{n}(1)=0$ demonstrate that

$$
\begin{aligned}
& \langle\partial u, v\rangle_{H}=\lim _{n \rightarrow \infty}\left\langle\partial u_{n}, v\right\rangle_{H}=\lim _{n \rightarrow \infty}\left(\lim _{m \rightarrow \infty}\left\langle\partial u_{n}, v_{m}\right\rangle_{H}\right) \\
& =\lim _{n \rightarrow \infty}\left(\lim _{m \rightarrow \infty} \int_{(0,1)}\left(\mathbf{u}_{n}\right)^{\prime}(x) \mathbf{v}_{m}(x) d x\right)=-\lim _{n \rightarrow \infty}\left(\lim _{m \rightarrow \infty} \int_{(0,1)} \mathbf{u}_{n}(x)\left(\mathbf{v}_{m}\right)^{\prime}(x) d x\right) \\
& =-\lim _{n \rightarrow \infty}\left(\lim _{m \rightarrow \infty}\left\langle u_{n}, \partial v_{m}\right\rangle_{H}\right)=-\lim _{n \rightarrow \infty}\left\langle u_{n}, \partial v\right\rangle_{H}=-\langle u, \partial v\rangle_{H} .
\end{aligned}
$$

The proof of Lemma 4.4 is thus completed.

Lemma 4.5. Assume Setting 4.1. Then

(i) it holds that $H_{1} \subseteq W^{2,2}((0,1), \mathbb{R})$ continuously and

(ii) it holds that

$$
\sup _{v \in H_{1} \backslash\{0\}}\left[\frac{\|v\|_{H_{1}}}{\|v\|_{W^{2,2}((0,1), \mathbb{R})}}+\frac{\|v\|_{W^{2,2}((0,1), \mathbb{R})}}{\|v\|_{H_{1}}}\right]<\infty .
$$


Proof of Lemma 4.5. First, observe that the fact that

$$
D(-A)=\left(W_{0}^{1,2}((0,1), \mathbb{R})\right) \cap\left(W^{2,2}((0,1), \mathbb{R})\right)
$$

(cf., e.g., Lunardi [30, Example 4.34] and Sell \& You [37, Section 3.8.1]) and the fact that $D(-A)=$ $H_{1}$ prove that

$$
H_{1} \subseteq W^{2,2}((0,1), \mathbb{R}) .
$$

Hence, we obtain that for every $v \in H_{1}$ it holds that $\partial v \in W^{1,2}((0,1), \mathbb{R})$. The fact that for every $n \in \mathbb{N}$ it holds that $e_{n} \in W_{0}^{1,2}\left((0,1), \mathbb{R}\right.$ ) and Lemma 4.4 (with $u=e_{n}, v=\partial v$ for $n \in \mathbb{N}, v \in H_{1}$ in the notation of Lemma 4.4) therefore prove that for every $v \in H_{1}$ it holds that

$$
\sum_{n=1}^{\infty}\left|\left\langle e_{n}, \partial^{2} v\right\rangle_{H}\right|^{2}=\sum_{n=1}^{\infty}\left|-\left\langle\partial e_{n}, \partial v\right\rangle_{H}\right|^{2}
$$

Furthermore, note that item (iii) of Lemma 4.3 assures that for every $v \in H_{1}$ it holds that $v \in$ $W_{0}^{1,2}((0,1), \mathbb{R})$. Combining (96) , the fact that for every $n \in \mathbb{N}$ it holds that $\partial e_{n} \in W^{1,2}((0,1), \mathbb{R})$, (95)), and Lemma 4.4 (with $u=v, v=\partial e_{n}$ for $n \in \mathbb{N}, v \in H_{1}$ in the notation of Lemma 4.4) hence shows that for every $v \in H_{1}$ it holds that

$$
\sum_{n=1}^{\infty}\left|\left\langle e_{n}, \partial^{2} v\right\rangle_{H}\right|^{2}=\sum_{n=1}^{\infty}\left|\left\langle\partial^{2} e_{n}, v\right\rangle_{H}\right|^{2}=\sum_{n=1}^{\infty}|\pi n|^{4}\left|\left\langle e_{n}, v\right\rangle_{H}\right|^{2}=\frac{1}{\left|c_{0}\right|^{2}}\|v\|_{H_{1}}^{2}<\infty
$$

This proves that for every $v \in H_{1}$ it holds that $\partial^{2} v \in H$ and

$$
\|v\|_{H_{1}}=c_{0}\left\|\partial^{2} v\right\|_{H}
$$

The fact that for every $v \in W^{2,2}((0,1), \mathbb{R})$ it holds that $\|v\|_{W^{2,2}((0,1), \mathbb{R})}^{2}=\|v\|_{H}^{2}+\|\partial v\|_{H}^{2}+\left\|\partial^{2} v\right\|_{H}^{2}$ and (95) hence ensure that for every $v \in H_{1}$ it holds that

$$
\|v\|_{H_{1}}=c_{0}\left\|\partial^{2} v\right\|_{H} \leq c_{0}\|v\|_{W^{2,2}((0,1), \mathbb{R})} .
$$

Next note that item (iii) of Lemma 4.3 and Poincaré's inequality (see, e.g., Brezis [5, Proposition 8.13]) imply that there exists $C \in(0, \infty)$ such that for every $v \in H_{1 / 2}$ it holds that $\|v\|_{W^{1,2}((0,1), \mathbb{R})} \leq C\|\partial v\|_{H}$. Combining this, (95), and (98) proves that there exists $C \in(0, \infty)$ such that for every $v \in H_{1}$ it holds that

$$
\|v\|_{W^{2,2}((0,1), \mathbb{R})}^{2}=\|v\|_{W^{1,2}((0,1), \mathbb{R})}^{2}+\left\|\partial^{2} v\right\|_{H}^{2} \leq C^{2}\|\partial v\|_{H}^{2}+\frac{1}{\left|c_{0}\right|^{2}}\|v\|_{H_{1}}^{2} .
$$

Item (iv) of Lemma 4.3 hence shows that there exists $C \in(0, \infty)$ such that for every $v \in H_{1}$ it holds that $v \in W^{2,2}((0,1), \mathbb{R})$ and

$$
\|v\|_{W^{2,2}((0,1), \mathbb{R})}^{2} \leq \frac{C^{2}}{c_{0}}\|v\|_{H_{1 / 2}}^{2}+\frac{1}{\left|c_{0}\right|^{2}}\|v\|_{H_{1}}^{2} \leq\left[\frac{C^{2}}{\left|c_{0}\right|^{2}}+\frac{1}{\left|c_{0}\right|^{2}}\right]\|v\|_{H_{1}}^{2} .
$$

This establishes item (ii). Moreover, observe that item (ii) and (99) imply item (iii). The proof of Lemma 4.5 is thus completed.

Lemma 4.6. Assume Setting 4.1. Then 
(i) it holds for every $s \in[0,1]$ that $H_{s} \subseteq W^{2 s, 2}((0,1), \mathbb{R})$ continuously,

(ii) it holds for every $s \in[0,1 / 2] \backslash\{1 / 4\}$ that $H_{s} \subseteq W_{0}^{2 s, 2}((0,1), \mathbb{R})$ continuously, and

(iii) it holds for every $s \in[0,1 / 2] \backslash\{1 / 4\}$ that $W_{0}^{2 s, 2}((0,1), \mathbb{R}) \subseteq H_{s}$ continuously.

Proof of Lemma 4.6. Throughout this proof consider the notation in Triebel [38, Section 1.3.2 on page 24] (cf., e.g., Lunardi [30, Definition 1.2]). Note that item (ii) of Lemma 4.5] ensures that

$$
H_{1} \subseteq W^{2,2}((0,1), \mathbb{R})
$$

continuously. Furthermore, observe that, e.g., Triebel [38, the theorem in Section 1.18.10 on page 142] (cf., e.g., Lunardi [30, Theorem 4.36]) and the fact that $\forall s \in$ $[0, \infty):\left(D\left((-A)^{s}\right),\left\|(-A)^{s}(\cdot)\right\|_{H}\right)=\left(H_{s},\|\cdot\|_{H_{s}}\right)$ prove that for every $s \in(0,1)$ it holds that

$$
\left(H, H_{1}\right)_{s, 2}=(H, D(-A))_{s, 2}=D\left((-A)^{s}\right)=H_{s}
$$

and

$$
\sup _{x \in H_{s} \backslash\{0\}}\left(\frac{\|x\|_{\left(H, H_{1}\right)_{s, 2}}}{\|x\|_{H_{s}}}+\frac{\|x\|_{H_{s}}}{\|x\|_{\left(H, H_{1}\right)_{s, 2}}}\right)<\infty .
$$

This, (102), and, e.g., Lunardi [30, Theorem 1.6] imply that for every $s \in(0,1)$ it holds that

$$
H_{s} \subseteq\left(H, W^{2,2}((0,1), \mathbb{R})\right)_{s, 2}
$$

continuously. The fact that for every $s \in(0,1)$ it holds that

$$
\left(H, W^{2,2}((0,1), \mathbb{R})\right)_{s, 2} \subseteq W^{2 s, 2}((0,1), \mathbb{R})
$$

continuously (cf., e.g., Triebel [38, Definition 1 in Section 4.2.1 on page 310, Theorem 1 in Section 4.3.1 on page 317, item (a) in Theorem 1 in Section 4.4.2 on page 323, and Remark 2 in Section 4.4.2 on page 324]) hence establishes item (ii). Moreover, note that, e.g., Triebel [38, the theorem in Section 1.18 .10 on page 142] (cf., e.g., Lunardi [30, Theorem 4.36]) and the fact that $\forall s \in[0, \infty):\left(D\left((-A)^{s}\right),\left\|(-A)^{s}(\cdot)\right\|_{H}\right)=\left(H_{s},\|\cdot\|_{H_{s}}\right)$ prove that for every $s \in(0,1)$ it holds that

$$
\left(H, H_{1 / 2}\right)_{s, 2}=\left(H, D\left((-A)^{1 / 2}\right)\right)_{s, 2}=D\left((-A)^{s / 2}\right)=H_{s / 2}
$$

and

$$
\sup _{x \in H_{s} \backslash\{0\}}\left(\frac{\|x\|_{\left(H, H_{1 / 2}\right)_{s, 2}}}{\|x\|_{H_{s / 2}}}+\frac{\|x\|_{H_{s / 2}}}{\|x\|_{\left(H, H_{1 / 2}\right)_{s, 2}}}\right)<\infty .
$$

The fact that for every $s \in(0,1) \backslash\{1 / 2\}$ it holds that

$$
\left(H, W_{0}^{1,2}((0,1), \mathbb{R})\right)_{s, 2}=W_{0}^{s, 2}((0,1), \mathbb{R}),
$$

(cf., e.g., Triebel [38, Definition 1 and Definition 2 in Section 4.2.1 on page 310, the definition in Section 4.3.2 on page 317, item (c) in Theorem 1 and Theorem 2 in Section 4.3.2 on page 318, item (a) in Theorem 1 in Section 4.4.2 on page 323, and Remark 2 in Section 4.4.2 on page 324]), items (ii) and (iii) of Lemma 4.3, and, e.g., Lunardi [30, Theorem 1.6] therefore assure that for every $s \in(0,1) \backslash\{1 / 2\}$ it holds that

$$
H_{s / 2}=\left(H, H_{1 / 2}\right)_{s, 2}=\left(H, W_{0}^{1,2}((0,1), \mathbb{R})\right)_{s, 2}=W_{0}^{s, 2}((0,1), \mathbb{R})
$$


and

$$
\sup _{x \in H_{s / 2} \backslash\{0\}}\left(\frac{\|x\|_{H_{s / 2}}}{\|x\|_{W^{s, 2}((0,1), \mathbb{R})}}+\frac{\|x\|_{W^{s, 2}((0,1), \mathbb{R})}}{\|x\|_{H_{s / 2}}}\right)<\infty .
$$

This establishes items (iii) and (iii). The proof of Lemma 4.6 is thus completed.

Lemma 4.7. Let $s \in[0, \infty), q, r \in[s, \infty)$ satisfy $r+q-s>1 / 2$. Then

(i) it holds for every $f \in W^{q, 2}((0,1), \mathbb{R}), g \in W^{r, 2}((0,1), \mathbb{R})$ that $f g \in W^{s, 2}((0,1), \mathbb{R})$ and

(ii) it holds that

$$
\sup _{f \in W^{q, 2}((0,1), \mathbb{R}) \backslash\{0\}} \sup _{g \in W^{r, 2}((0,1), \mathbb{R}) \backslash\{0\}}\left[\frac{\|f g\|_{W^{s, 2}((0,1), \mathbb{R})}}{\|f\|_{W^{q, 2}((0,1), \mathbb{R})}\|g\|_{W^{r, 2}((0,1), \mathbb{R})}}\right]<\infty .
$$

Proof of Lemma 4.7. Observe that, e.g., Behzadan \& Holst [2, Theorem 7.5] (with $n=1, \Omega=$ $(0,1), s=s, p=2, s_{1}=q, s_{2}=r, p_{1}=2, p_{2}=2$ in the notation of Behzadan \& Holst [2, Theorem 7.5]) establishes items (ii) and (iii). The proof of Lemma 4.7 is thus completed.

Lemma 4.8. Assume Setting 4.1. Then

(i) there exists a unique bounded linear function $\bar{\partial}: H \rightarrow H_{-1 / 2}$ which satisfies for every $v \in$ $W^{1,2}((0,1), \mathbb{R})$ that $\bar{\partial} v=\partial v$ and

(ii) it holds that $\|\bar{\partial}\|_{L\left(H, H_{-1 / 2}\right)} \leq\left|c_{0}\right|^{-1 / 2}$.

Proof of Lemma 4.8. Observe that Lemma 4.4 and items (ii), (iii), and (iv) of Lemma 4.3 show that for every $v \in W^{1,2}((0,1), \mathbb{R})$ it holds that

$$
\begin{aligned}
\|\partial v\|_{H_{-1 / 2}} & =\sup _{u \in\left(H_{1 / 2} \backslash\{0\}\right)} \frac{\left|\langle\partial v, u\rangle_{H}\right|}{\|u\|_{H_{1 / 2}}}=\sup _{u \in\left(W_{0}^{1,2}((0,1), \mathbb{R}) \backslash\{0\}\right)} \frac{\left|\langle\partial v, u\rangle_{H}\right|}{\|u\|_{H_{1 / 2}}}=\sup _{u \in\left(W_{0}^{1,2}((0,1), \mathbb{R}) \backslash\{0\}\right)} \frac{\left|\langle v, \partial u\rangle_{H}\right|}{\|u\|_{H_{1 / 2}}} \\
& \leq \sup _{u \in\left(W_{0}^{1,2}((0,1), \mathbb{R}) \backslash\{0\}\right)} \frac{\|v\|_{H}\|\partial u\|_{H}}{\|u\|_{H_{1 / 2}}}=\left|c_{0}\right|^{-1 / 2} \sup _{u \in\left(H_{1 / 2} \backslash\{0\}\right)} \frac{\|v\|_{H}\|u\|_{H_{1 / 2}}}{\|u\|_{H_{1 / 2}}}=\left|c_{0}\right|^{-1 / 2}\|v\|_{H} .
\end{aligned}
$$

The fact that $W^{1,2}((0,1), \mathbb{R}) \subseteq H$ densely therefore establishes items (ii) and (ii). The proof of Lemma 4.8 is thus completed.

Lemma 4.9. Assume Setting [4.1] and let $\alpha \in[0,1 / 2]$. Then

$$
\sup _{v \in W^{1,2}((0,1), \mathbb{R}) \backslash\{0\}} \frac{\|\partial v\|_{H_{-\alpha}}}{\|v\|_{W^{1-2 \alpha, 2}((0,1), \mathbb{R})}}<\infty .
$$

Proof of Lemma 4.9. Throughout this proof consider the notation in Triebel [38, Section 1.3.2 on page 24] (cf., e.g., Lunardi [30, Definition 1.2]) and let $\bar{\partial}: H \rightarrow H_{-1 / 2}$ be the continuous linear function which satisfies for every $v \in W^{1,2}((0,1), \mathbb{R})$ that $\bar{\partial} v=\partial v$ (cf. item (ii) of Lemma 4.8). Observe that, e.g., Triebel [38, the theorem in Section 1.18.10 on page 142] (cf., e.g., Lunardi [30, Theorem 4.36]) and the fact that $\forall s \in[0, \infty):\left(D\left((-A)^{s}\right),\left\|(-A)^{s}(\cdot)\right\|_{H}\right)=\left(H_{s},\|\cdot\|_{H_{s}}\right)$ prove that for every $s \in(0,1)$ it holds that

$$
\left(H, H_{1 / 2}\right)_{s, 2}=\left(H, D\left((-A)^{1 / 2}\right)\right)_{s, 2}=D\left((-A)^{s / 2}\right)=H_{s / 2}
$$


and

$$
\sup _{x \in H_{1} \backslash\{0\}}\left(\frac{\|x\|_{\left(H, H_{1 / 2}\right)_{s, 2}}}{\|x\|_{H_{s / 2}}}+\frac{\|x\|_{H_{s / 2}}}{\|x\|_{\left(H, H_{1 / 2}\right)_{s, 2}}}\right)<\infty .
$$

The fact that for every $r \in[0, \infty)$ it holds that $\left(H_{r}\right)^{\prime}$ and $H_{-r}$ are isometrically isomorphic and, e.g., Triebel [38, item (b) of the theorem in Section 1.3.3 on page 25 and the theorem in Section 1.11.2 on page 69] (cf., e.g., Lunardi [30, Theorem 1.18]) hence imply that for every $s \in(0,1)$ it holds that

$$
\left(H_{-1 / 2}, H\right)_{s, 2}=H_{(s-1) / 2}
$$

and

$$
\sup _{x \in H_{(s-1) / 2} \backslash\{0\}}\left(\frac{\|x\|_{H_{(s-1) / 2}}}{\|x\|_{\left(H_{-1 / 2}, H\right)_{s, 2}}}+\frac{\|x\|_{\left(H_{-1 / 2}, H\right)_{s, 2}}}{\|x\|_{H_{(s-1) / 2}}}\right)<\infty .
$$

In addition, note that, e.g., Triebel [38, Definition 1 in Section 4.2.1 on page 310, Theorem 1 in Section 4.3.1 on page 317, item (a) in Theorem 1 in Section 4.4.2 on page 323, and Remark 2 in Section 4.4 .2 on page 324] ensures that for every $s \in(0,1)$ it holds that

$$
\left(H, W^{1,2}((0,1), \mathbb{R})\right)_{s, 2}=W^{s, 2}((0,1), \mathbb{R})
$$

and

$$
\sup _{x \in W^{s, 2}((0,1), \mathbb{R}) \backslash\{0\}}\left(\frac{\|x\|_{W^{s, 2}((0,1), \mathbb{R})}}{\|x\|_{\left(H, W^{1,2}((0,1), \mathbb{R})\right)_{s, 2}}}+\frac{\|x\|_{\left(H, W^{1,2}((0,1), \mathbb{R})\right)_{s, 2}}}{\|x\|_{W^{s, 2}((0,1), \mathbb{R})}}\right)<\infty .
$$

Furthermore, observe that item (iii) of Lemma 4.8 ensures that for every $v \in H$ it holds that

$$
\|\bar{\partial} v\|_{H_{-1 / 2}} \leq\left|c_{0}\right|^{-1 / 2}\|v\|_{H} .
$$

Combining this, the fact that for every $v \in W^{1,2}((0,1), \mathbb{R})$ it holds that

$$
\|\partial v\|_{H} \leq\|v\|_{W^{1,2}((0,1), \mathbb{R})}
$$

(117) -(120), and, e.g, Lunardi [30, Theorem 1.6] establishes (114). The proof of Lemma 4.9 is thus completed.

Lemma 4.10. Assume Setting 4.1 and let $\alpha \in(1 / 4, \infty)$. Then it holds for every $v \in H_{\alpha+(1 / 2)}$ that

$$
\|\partial v\|_{L^{\infty}(\lambda ; \mathbb{R})} \leq \sqrt{2}\left|c_{0}\right|^{-\alpha-(1 / 2)}\|v\|_{H_{\alpha+(1 / 2)}} \sqrt{\sum_{n=1}^{\infty}|\pi n|^{-4 \alpha}} .
$$

Proof of Lemma 4.10. Note that the fact that $\forall v \in H_{1 / 2}: \sum_{n=1}^{\infty}\left|\mathfrak{v}_{e_{n}}\right|\left|\left\langle e_{n}, v\right\rangle_{H}\right|^{2}=\left\|(-A)^{1 / 2} v\right\|_{H}^{2}=$ $\|v\|_{H_{1 / 2}}^{2}<\infty$ shows that for every $v \in H_{1 / 2}$ it holds that

$$
\limsup _{N \rightarrow \infty}\left\|v-\sum_{n=1}^{N}\left\langle e_{n}, v\right\rangle_{H} e_{n}\right\|_{H_{1 / 2}}^{2}=\limsup _{N \rightarrow \infty}\left[\sum_{n=N+1}^{\infty}\left|\mathfrak{v}_{e_{n}} \|\left\langle e_{n}, v\right\rangle_{H}\right|^{2}\right]=0 .
$$

In addition, observe that items (iii) and (iv) of Lemma 4.3 ensure that $\left(H_{1 / 2} \ni u \mapsto \partial u \in H\right) \in$ $L\left(H_{1 / 2}, H\right)$. Combining (124) and the Cauchy-Schwarz inequality hence implies that for every 
$v \in H_{\alpha+(1 / 2)}$ it holds that

$$
\begin{aligned}
& \|\partial v\|_{L^{\infty}(\lambda ; \mathbb{R})}=\left\|\partial\left(\sum_{n=1}^{\infty}\left\langle e_{n}, v\right\rangle_{H} e_{n}\right)\right\|_{L^{\infty}(\lambda ; \mathbb{R})}=\left\|\sum_{n=1}^{\infty}\left\langle e_{n}, v\right\rangle_{H} \partial e_{n}\right\|_{L^{\infty}(\lambda ; \mathbb{R})} \\
& \leq \sum_{n=1}^{\infty}\left|\left\langle e_{n}, v\right\rangle_{H}\right|\left\|\partial e_{n}\right\|_{L^{\infty}(\lambda ; \mathbb{R})} \\
& \leq \sup _{n \in \mathbb{N}}\left(\left|c_{0} \pi^{2} n^{2}\right|^{-1 / 2}\left\|\partial e_{n}\right\|_{L^{\infty}(\lambda ; \mathbb{R})}\right)\left[\sum_{n=1}^{\infty}\left|\left\langle e_{n}, v\right\rangle_{H}\right|\left|c_{0} \pi^{2} n^{2}\right|^{1 / 2}\right] \\
& \leq \sup _{n \in \mathbb{N}}\left(\left|c_{0} \pi^{2} n^{2}\right|^{-1 / 2} \sqrt{2} n \pi\right) \sqrt{\sum_{n=1}^{\infty}\left|\left\langle e_{n}, v\right\rangle_{H}\right|^{2}\left|c_{0} \pi^{2} n^{2}\right|^{1+2 \alpha}} \sqrt{\sum_{n=1}^{\infty}\left|c_{0} \pi^{2} n^{2}\right|^{-2 \alpha}} \\
& =\sqrt{2}\left|c_{0}\right|^{-\alpha-(1 / 2)}\|v\|_{H_{\alpha+(1 / 2)}} \sqrt{\sum_{n=1}^{\infty}|\pi n|^{-4 \alpha}} .
\end{aligned}
$$

The proof of Lemma 4.10 is thus completed.

Lemma 4.11. Let $\lambda: \mathcal{B}((0,1)) \rightarrow[0,1]$ be the Lebesgue-Borel measure on $(0,1)$ and let $q, r \in$ $[1, \infty), \alpha \in(0,1)$ satisfy $\alpha\left(\frac{1}{q}+1-\frac{1}{r}\right)=\frac{1}{q}$. Then there exists $C \in(0, \infty)$ such that for every $u \in W_{0}^{1, r}((0,1), \mathbb{R})$ it holds that

$$
\|u\|_{L^{\infty}(\lambda ; \mathbb{R})} \leq C\|u\|_{W^{1, r}((0,1), \mathbb{R})}^{\alpha}\|u\|_{L^{q}(\lambda ; \mathbb{R})}^{1-\alpha} .
$$

Proof of Lemma 4.11. Throughout this proof let $p \in \mathbb{R}$ satisfy $q=p\left(\frac{1}{\alpha}-1\right)$, for every function $f:(0,1) \rightarrow \mathbb{R}$ let $[f]_{\lambda, \mathcal{B}(\mathbb{R})}$ be the set given by

$$
[f]_{\lambda, \mathcal{B}(\mathbb{R})}=\left\{g:(0,1) \rightarrow \mathbb{R}:\left[\begin{array}{l}
(\exists D \in \mathcal{B}((0,1)):[\lambda(D)=0 \text { and }\{t \in(0,1): f(t) \neq g(t)\} \subseteq D]) \\
\text { and }\left(\forall D \in \mathcal{B}(\mathbb{R}): g^{-1}(D) \in \mathcal{B}((0,1))\right)
\end{array}\right]\right\}
$$

let $():.\left\{[v]_{\lambda, \mathcal{B}(\mathbb{R})}:(v:(0,1) \rightarrow \mathbb{R}\right.$ is uniformly continous $\left.)\right\} \rightarrow \mathcal{C}([0,1], \mathbb{R})$ be the function which satisfies for every $v \in \mathcal{C}([0,1], \mathbb{R})$ that

$$
\underline{\left[\left.v\right|_{(0,1)}\right]_{\lambda, \mathcal{B}(\mathbb{R})}}=v,
$$

for every $u \in W^{1, r}((0,1), \mathbb{R})$ let $\partial u \in L^{r}(\lambda ; \mathbb{R})$ satisfy for every $\varphi \in \mathcal{C}_{c p t}^{\infty}((0,1), \mathbb{R}), v \in \mathcal{L}^{r}(\lambda ; \mathbb{R})$ with $v \in \partial u$ that $\int_{(0,1)} \underline{u}(x) \varphi^{\prime}(x) d x=-\int_{(0,1)} v(x) \varphi(x) d x$, and let $G: \mathbb{R} \rightarrow \mathbb{R}$ be the function which satisfies for every $t \in \mathbb{R}$ that $G(t)=|t|^{\frac{1}{\alpha}-1} t$. Note that

(a) it holds that $G(0)=0$,

(b) it holds that $G \in \mathcal{C}^{1}(\mathbb{R}, \mathbb{R})$, and

(c) it holds for every $t \in[0,1]$ that $G^{\prime}(t)=\frac{1}{\alpha}|t|^{\frac{1}{\alpha}-1}$.

This and, e.g., Brezis [5, Corollary 8.1] show that for every $u \in W^{1, r}((0,1), \mathbb{R})$ it holds that

$$
\left[(G(\underline{u}(x)))_{x \in(0,1)}\right]_{\lambda, \mathcal{B}(\mathbb{R})} \in W^{1, r}((0,1), \mathbb{R})
$$


and

$$
\partial\left[(G(\underline{u}(x)))_{x \in(0,1)}\right]_{\lambda, \mathcal{B}(\mathbb{R})}=\left[\left(G^{\prime}(\underline{u}(x))\right)_{x \in(0,1)}\right]_{\lambda, \mathcal{B}(\mathbb{R})} \partial u .
$$

Combining this and, e.g., Brezis [5, Theorem 8.2] ensures that for every $u \in W^{1, r}((0,1), \mathbb{R})$, $v \in \mathcal{L}^{r}(\lambda ; \mathbb{R}), x \in[0,1]$ with $v \in \partial u$ it holds that

$$
G(\underline{u}(x))=G(\underline{u}(0))+\int_{0}^{x} G^{\prime}(\underline{u}(t)) v(t) d t .
$$

This implies that for every $u \in W^{1, r}((0,1), \mathbb{R}), v \in \mathcal{L}^{r}(\lambda ; \mathbb{R})$ with $v \in \partial u, \underline{u}(0)=0$ it holds that

$$
\begin{aligned}
& \|u\|_{L^{\infty}(\lambda ; \mathbb{R})}^{\frac{1}{\alpha}}=\sup _{x \in[0,1]}|G(\underline{u}(x))| \leq \int_{0}^{1}\left|G^{\prime}(\underline{u}(t)) v(t)\right| d t \\
& =\frac{1}{\alpha} \int_{0}^{1}|\underline{u}(t)|^{\frac{1}{\alpha}-1}|v(t)| d t=\frac{1}{\alpha}\left\||u|^{\frac{1}{\alpha}-1}|\partial u|\right\|_{L^{1}(\lambda ; \mathbb{R})} .
\end{aligned}
$$

Next observe that the fact that $\frac{1}{r}=\frac{1}{q}+1-\frac{1}{q \alpha}$ and the fact that $\frac{1}{p}=\frac{1}{q \alpha}-\frac{1}{q}$ ensure that $\frac{1}{p}+\frac{1}{r}=1$. Combining this with (132) and Hölder's inequality demonstrates that for every $u \in W_{0}^{1, r}((0,1), \mathbb{R})$ it holds that

$$
\|u\|_{L^{\infty}(\lambda ; \mathbb{R})}^{\frac{1}{\alpha}} \leq \frac{1}{\alpha}\left\||u|^{\frac{1}{\alpha}-1}\right\|_{L^{p}(\lambda ; \mathbb{R})}\|\partial u\|_{L^{r}(\lambda ; \mathbb{R})} \leq \frac{1}{\alpha}\|u\|_{L^{p\left(\frac{1}{\alpha}-1\right)}(\lambda ; \mathbb{R})}^{\frac{1-\alpha}{\alpha}}\|\partial u\|_{L^{r}(\lambda ; \mathbb{R})} .
$$

This completes the proof of Lemma 4.11.

Lemma 4.12. Let $\lambda: \mathcal{B}((0,1)) \rightarrow[0,1]$ be the Lebesgue-Borel measure on $(0,1)$ and let $q \in[1, \infty)$, $p \in(q, \infty), r \in(1, \infty), \alpha \in(0,1)$ satisfy $\alpha\left(\frac{1}{q}+1-\frac{1}{r}\right)=\frac{1}{q}-\frac{1}{p}$. Then there exists $C \in(0, \infty)$ such that for every $u \in W_{0}^{1, r}((0,1), \mathbb{R})$ it holds that

$$
\|u\|_{L^{p}(\lambda ; \mathbb{R})} \leq C\|u\|_{W^{1, r}((0,1), \mathbb{R})}^{\alpha}\|u\|_{L^{q}(\lambda ; \mathbb{R})}^{1-\alpha} .
$$

Proof of Lemma 4.12. Throughout this proof let $\beta=\frac{\alpha p}{p-q}$. Note that Hölder's inequality proves that for every $u \in W^{1, r}((0,1), \mathbb{R})$ it holds that

$$
\|u\|_{L^{p}(\lambda ; \mathbb{R})}^{p}=\left\||u|^{q}|u|^{p-q}\right\|_{L^{1}(\lambda ; \mathbb{R})} \leq\|u\|_{L^{q}(\lambda ; \mathbb{R})}^{q}\|u\|_{L^{\infty}(\lambda ; \mathbb{R})}^{p-q} .
$$

Lemma 4.11 (with $q=q, r=r, \alpha=\beta$ in the notation of Lemma 4.11) hence shows that there exists $C \in(0, \infty)$ such that for every $u \in W_{0}^{1, r}((0,1), \mathbb{R})$ it holds that

$$
\begin{aligned}
\|u\|_{L^{p}(\lambda ; \mathbb{R})}^{p} & \leq C^{p}\|u\|_{L^{q}(\lambda ; \mathbb{R})}^{q}\|u\|_{W^{1, r}((0,1), \mathbb{R})}^{\beta(p-q)}\|u\|_{L^{q}(\lambda ; \mathbb{R})}^{(1-\beta)(p-q)} \\
& =C^{p}\|u\|_{L^{q}(\lambda ; \mathbb{R})}^{p-\beta p+\beta q}\|u\|_{W^{1, r}((0,1), \mathbb{R})}^{\beta(p-q)} .
\end{aligned}
$$

This implies that there exists $C \in(0, \infty)$ such that for every $u \in W_{0}^{1, r}((0,1), \mathbb{R})$ it holds that

$$
\|u\|_{L^{p}(\lambda ; \mathbb{R})} \leq C\|u\|_{L^{q}(\lambda ; \mathbb{R})}^{1-\beta\left(1-\frac{q}{p}\right)}\|u\|_{W^{1, r}((0,1), \mathbb{R})}^{\beta\left(1-\frac{q}{p}\right)}=C\|u\|_{L^{q}(\lambda ; \mathbb{R})}^{1-\alpha}\|u\|_{W^{1, r}((0,1), \mathbb{R})}^{\alpha} .
$$

The proof of Lemma 4.12 is thus completed. 


\subsection{Analysis of the nonlinearity}

In this subsection we recall in Lemmas 4.134.17, Corollary 4.18, Lemmas 4.194.21, Corollary 4.22, Lemma 4.23, and Corollary 4.24 below a few elementary and well-known properties of the nonlinearity appearing in the stochastic Burgers equation. Corollaries 4.22 and 4.24 are then used in Section 5 below to establish in Theorem 5.10 the main result of this article.

Lemma 4.13. Assume Setting 4.1. Then

(i) it holds for every $u \in H_{1 / 2}$ that $u^{2} \in W^{1,2}((0,1), \mathbb{R})$ and $u \partial u=\frac{1}{2} \partial\left(u^{2}\right)$,

(ii) it holds for every $v, w \in H_{1 / 2}$ that

$$
\|F(v)-F(w)\|_{H} \leq \frac{\left|c_{1}\right|}{\sqrt{3} c_{0}}\left(\|v\|_{H_{1 / 2}}+\|w\|_{H_{1 / 2}}\right)\|v-w\|_{H_{1 / 2}},
$$

(iii) it holds that $F \in \mathcal{C}^{1}\left(H_{1 / 2}, H\right)$, and

(iv) it holds for every $v, w \in H_{1 / 2}$ that $F^{\prime}(v) w=c_{1}(w \partial v+v \partial w)$.

Proof of Lemma 4.13. Observe that items (iii) and (iii) of Lemma 4.3 and, e.g., [20, Lemma 4.5] imply item (ii). Furthermore, note that for every $v, w \in H_{1 / 2}$ it holds that

$$
\|F(v)-F(w)\|_{H} \leq\left|c_{1}\right|\|\partial v\|_{H}\|v-w\|_{L^{\infty}(\lambda ; \mathbb{R})}+\left|c_{1}\right|\|w\|_{L^{\infty}(\lambda ; \mathbb{R})}\|\partial(v-w)\|_{H} .
$$

Items (iv) and (清) of Lemma 4.3 therefore show that for every $v, w \in H_{1 / 2}$ it holds that

$$
\|F(v)-F(w)\|_{H} \leq \frac{\left|c_{1}\right|}{\sqrt{3} c_{0}}\left(\|v\|_{H_{1 / 2}}\|v-w\|_{H_{1 / 2}}+\|w\|_{H_{1 / 2}}\|v-w\|_{H_{1 / 2}}\right) .
$$

This establishes item (iii). In addition, note that for every $v, w \in H_{1 / 2}$ it holds that

$$
F(v+w)-F(v)=c_{1}((v+w)(\partial v+\partial w)-v \partial v)=c_{1}(v \partial w+w \partial v+w \partial w) .
$$

Items (iv) and ( (v) of Lemma 4.3 hence imply that for every $v, w \in H_{1 / 2}$ it holds that

$$
\begin{aligned}
\left\|F(v+w)-F(v)-c_{1}(v \partial w+w \partial v)\right\|_{H} & =\left\|c_{1} w \partial w\right\|_{H} \\
& \leq\left\|c_{1} w\right\|_{L^{\infty}(\lambda ; \mathbb{R})}\|\partial w\|_{H} \leq \frac{\left|c_{1}\right|}{\sqrt{3} c_{0}}\|w\|_{H_{1 / 2}}^{2} .
\end{aligned}
$$

Therefore, we obtain that

(a) it holds that $F: H_{1 / 2} \rightarrow H$ is differentiable and

(b) it holds for every $v, w \in H_{1 / 2}$ that $F^{\prime}(v) w=c_{1}(w \partial v+v \partial w)$.

Items (iv) and ( (v) of Lemma 4.3 hence assure that for every $u, v \in H_{1 / 2}$ it holds that

$$
\begin{aligned}
& \left\|F^{\prime}(u)-F^{\prime}(v)\right\|_{L\left(H_{1 / 2}, H\right)}=\left|c_{1}\right| \sup _{w \in H_{1 / 2} \backslash\{0\}} \frac{\|u \partial w+w \partial u-(v \partial w+w \partial v)\|_{H}}{\|w\|_{H_{1 / 2}}} \\
& \leq\left|c_{1}\right| \sup _{w \in H_{1 / 2} \backslash\{0\}} \frac{\|u \partial w-v \partial w\|_{H}+\|w \partial u-w \partial v\|_{H}}{\|w\|_{H_{1 / 2}}} \\
& \leq\left|c_{1}\right| \sup _{w \in H_{1 / 2} \backslash\{0\}} \frac{\|u-v\|_{L^{\infty}(\lambda ; \mathbb{R})}\|\partial w\|_{H}+\|w\|_{L^{\infty}(\lambda ; \mathbb{R})}\|\partial(u-v)\|_{H}}{\|w\|_{H_{1 / 2}}} \leq \frac{2\left|c_{1}\right|}{\sqrt{3} c_{0}}\|u-v\|_{H_{1 / 2}} .
\end{aligned}
$$

Combining items (国) and (b) therefore establishes items (iii) and (iv). The proof of Lemma 4.13 is thus completed. 
Lemma 4.14. Let $\left(X, d_{X}\right)$ be a metric space, let $\left(Y, d_{Y}\right)$ be a complete metric space, let $S \subseteq X$ be a dense subset, and let $F: S \rightarrow Y$ be a locally uniformly continuous function. Then there exists a unique continuous function $\bar{F}: X \rightarrow Y$ which satisfies for every $x \in S$ that $\bar{F}(x)=F(x)$.

Proof of Lemma 4.14. Throughout this proof let $U_{x} \subseteq X, x \in S$, be non-empty open sets which satisfy that

(a) it holds for every $x \in S$ that $\left.F\right|_{U_{x} \cap S}: U_{x} \cap S \rightarrow Y$ is uniformly continuous and

(b) it holds for every $x \in S$ that $x \in U_{x}$.

Observe that the fact that for every $x \in S$ it holds that $U_{x} \cap S$ is a dense subset of $U_{x}$ and, e.g., Searcóid [36, Theorem 10.9.1] show that there exist unique uniformly continuous functions $F_{x}: U_{x} \rightarrow Y, x \in S$, which satisfy for every $x \in S, u \in\left(U_{x} \cap S\right)$ that

$$
F_{x}(u)=F(u) .
$$

Note that (144) and the fact that for every $x, \mathbf{x} \in S$ with $\left(U_{x} \cap U_{\mathbf{x}}\right) \neq \emptyset$ it holds that $\left(U_{x} \cap U_{\mathbf{x}}\right) \cap S$ is a dense subset of $\left(U_{x} \cap U_{\mathbf{x}}\right)$ ensure that for every $x, \mathbf{x} \in S, u \in\left(U_{x} \cap U_{\mathbf{x}}\right)$ there exist $\left(u_{n}\right)_{n \in \mathbb{N}} \subseteq$ $\left(U_{x} \cap U_{\mathbf{x}} \cap S\right)$ such that $\lim \sup _{n \rightarrow \infty}\left\|u-u_{n}\right\|_{X}=0$ and

$$
\left\|F_{x}(u)-F_{\mathbf{x}}(u)\right\|_{Y}=\limsup _{n \rightarrow \infty}\left\|F_{x}\left(u_{n}\right)-F_{\mathbf{x}}\left(u_{n}\right)\right\|_{Y}=\limsup _{n \rightarrow \infty}\left\|F\left(u_{n}\right)-F\left(u_{n}\right)\right\|_{Y}=0 .
$$

This proves that for every $x, \mathbf{x} \in S, u \in\left(U_{x} \cap U_{\mathbf{x}}\right)$ it holds that

$$
F_{x}(u)=F_{\mathbf{x}}(u)
$$

Moreover, observe that the assumption that $S \subseteq X$ is a dense subset ensures that $X=\cup_{x \in S} U_{x}$. Combining (144) and (146) hence shows that there exists a unique continuous function $\bar{F}: X \rightarrow Y$ which satisfies for every $u \in S$ that

$$
\bar{F}(u)=F(u) .
$$

The proof of Lemma 4.14 is thus completed.

Lemma 4.15. Assume Setting 4.1] and let $\gamma \in\left(\frac{1}{8}, \frac{1}{2}\right], \nu \in\left(\left[\frac{1}{2}-\gamma, \frac{1}{2}\right] \cap\left(\frac{3}{4}-2 \gamma, \infty\right)\right)$. Then there exists $C \in \mathbb{R}$ such that for every $v, w \in H_{1 / 2}$ it holds that

$$
\|F(v)-F(w)\|_{H_{-\nu}} \leq C\|v-w\|_{H_{\gamma}}\left(1+\|v\|_{H_{\gamma}}+\|w\|_{H_{\gamma}}\right) .
$$

Proof of Lemma 4.15. Note that the fact that $\nu>\frac{3}{4}-2 \gamma$ ensures that $(2 \gamma)+(2 \gamma)-(1-2 \nu)>\frac{1}{2}$. Combining this, Lemma 4.7 (with $s=1-2 \nu, q=2 \gamma, r=2 \gamma$ in the notation of Lemma 4.7), Lemma 4.9 (with $\alpha=\nu$ in the notation of Lemma 4.9), and item (ii) of Lemma 4.13 shows that there exists $C \in[1, \infty)$ such that for every $v, w \in H_{1 / 2}$ it holds that $\left(v^{2}-w^{2}\right) \in W^{1,2}((0,1), \mathbb{R})$ and

$$
\begin{aligned}
\left\|\partial\left(v^{2}-w^{2}\right)\right\|_{H_{-\nu}} & \leq C\left\|v^{2}-w^{2}\right\|_{W^{1-2 \nu, 2}((0,1), \mathbb{R})} \\
& \leq C^{2}\|v-w\|_{W^{2 \gamma, 2}((0,1), \mathbb{R})}\|v+w\|_{W^{2 \gamma, 2}((0,1), \mathbb{R})} .
\end{aligned}
$$

Item (ii) of Lemma 4.6 hence proves that there exists $C \in \mathbb{R}$ such that for every $v, w \in H_{1 / 2}$ it holds that

$$
\left\|\partial\left(v^{2}-w^{2}\right)\right\|_{H_{-\nu}} \leq C\|v-w\|_{H_{\gamma}}\left(\|v\|_{H_{\gamma}}+\|w\|_{H_{\gamma}}\right)
$$

Item (ii) of Lemma 4.13 therefore establishes (148). The proof of Lemma 4.15 is thus completed. 
Lemma 4.16. Assume Setting [4.1 and let $\gamma \in\left(\frac{1}{8}, \frac{1}{2}\right], \nu \in\left(\left[\frac{1}{2}-\gamma, \frac{1}{2}\right] \cap\left(\frac{3}{4}-2 \gamma, \infty\right)\right)$. Then

(i) there exists a unique continuous function $\bar{F}: H_{\gamma} \rightarrow H_{-\nu}$ which satisfies for every $v \in H_{1 / 2}$ that $\bar{F}(v)=F(v)$ and

(ii) there exists $C \in \mathbb{R}$ which satisfies for every $v, w \in H_{\gamma}$ that

$$
\|\bar{F}(v)-\bar{F}(w)\|_{H_{-\nu}} \leq C\|v-w\|_{H_{\gamma}}\left(1+\|v\|_{H_{\gamma}}+\|w\|_{H_{\gamma}}\right) .
$$

Proof of Lemma 4.16. Observe that Lemma 4.15 (with $\gamma=\gamma, \nu=\nu$ in the notation of Lemma 4.15) ensures that there exists $C \in \mathbb{R}$ such that for every $v, w \in H_{1 / 2}$ it holds that

$$
\|F(v)-F(w)\|_{H_{-\nu}} \leq C\|v-w\|_{H_{\gamma}}\left(1+\|v\|_{H_{\gamma}}+\|w\|_{H_{\gamma}}\right) .
$$

Lemma 4.14 (with $X=H_{\gamma}, d_{X}=\left(\left(H_{\gamma} \times H_{\gamma}\right) \ni\left(h_{1}, h_{2}\right) \mapsto\left\|h_{1}-h_{2}\right\|_{H_{\gamma}} \in[0, \infty)\right), Y=H_{-\nu}$, $d_{Y}=\left(\left(H_{-\nu} \times H_{-\nu}\right) \ni\left(h_{1}, h_{2}\right) \mapsto\left\|h_{1}-h_{2}\right\|_{H_{-\nu}} \in[0, \infty)\right), S=H_{1 / 2}, F=F$ in the notation of Lemma 4.14) therefore establishes item (ii). Moreover, note that the fact that $H_{1 / 2} \subseteq H_{\gamma}$ continuously and densely ensures that for every $v \in H_{\gamma}$ there exist $\left(v_{n}\right)_{n \in \mathbb{N}} \subseteq H_{1 / 2}$ such that $\lim \sup _{n \rightarrow \infty}\left\|v-v_{n}\right\|_{H_{\gamma}}=0$. Item (ii) therefore implies that for every $v, w \in H_{\gamma}$ there exist $\left(v_{n}\right)_{n \in \mathbb{N}} \subseteq H_{1 / 2}$ and $\left(w_{n}\right)_{n \in \mathbb{N}} \subseteq H_{1 / 2}$ such that $\lim \sup _{n \rightarrow \infty}\left(\left\|v-v_{n}\right\|_{H_{\gamma}}+\left\|w-w_{n}\right\|_{H_{\gamma}}\right)=0$ and

$$
\begin{aligned}
\|\bar{F}(v)-\bar{F}(w)\|_{H_{-\nu} \leq} \leq & \lim \sup _{n \rightarrow \infty}\left\|\bar{F}(v)-\bar{F}\left(v_{n}\right)\right\|_{H_{-\nu}}+\lim \sup _{n \rightarrow \infty}\left\|\bar{F}\left(v_{n}\right)-\bar{F}\left(w_{n}\right)\right\|_{H_{-\nu}} \\
& +\lim \sup _{n \rightarrow \infty}\left\|\bar{F}\left(w_{n}\right)-\bar{F}(w)\right\|_{H_{-\nu}} \\
= & \limsup _{n \rightarrow \infty}\left\|\bar{F}\left(v_{n}\right)-\bar{F}\left(w_{n}\right)\right\|_{H_{-\nu}} \\
= & \limsup _{n \rightarrow \infty}\left\|F\left(v_{n}\right)-F\left(w_{n}\right)\right\|_{H_{-\nu}} .
\end{aligned}
$$

Combining this and (152) shows that there exists $C \in \mathbb{R}$ such that for every $v, w \in H_{\gamma}$ there exist $\left(v_{n}\right)_{n \in \mathbb{N}} \subseteq H_{1 / 2}$ and $\left(w_{n}\right)_{n \in \mathbb{N}} \subseteq H_{1 / 2}$ such that

$$
\begin{aligned}
\|\bar{F}(v)-\bar{F}(w)\|_{H_{-\nu}} & \leq C \lim \sup _{n \rightarrow \infty}\left(\left\|v_{n}-w_{n}\right\|_{H_{\gamma}}\left(1+\left\|v_{n}\right\|_{H_{\gamma}}+\left\|w_{n}\right\|_{H_{\gamma}}\right)\right) \\
& =C\|v-w\|_{H_{\gamma}}\left(1+\|v\|_{H_{\gamma}}+\|w\|_{H_{\gamma}}\right) .
\end{aligned}
$$

This establish item (iii). The proof of Lemma 4.16 is thus completed.

Lemma 4.17. Assume Setting 4.1 and let $\bar{\partial}: H \rightarrow H_{-1 / 2}$ be the continuous function which satisfies for every $v \in W^{1,2}((0,1), \mathbb{R})$ that $\bar{\partial} v=\partial v$ (cf. item (ii) of Lemma 4.8). Then there exists $C \in \mathbb{R}$ such that for every $v, w \in H_{1 / 8}$ it holds that $\left(v^{2}-w^{2}\right) \in H$ and

$$
\left\|\bar{\partial}\left(v^{2}-w^{2}\right)\right\|_{H_{-1 / 2}} \leq C\|v-w\|_{H_{1 / 8}}\left(1+\|v\|_{H_{1 / 8}}+\|w\|_{H_{1 / 8}}\right) .
$$

Proof of Lemma 4.17. Note that item (ii) of Lemma 4.6 (with $s=1 / 8$ in the notation of item (ii) of Lemma 4.6) ensures that $H_{1 / 8} \subseteq W^{1 / 4,2}((0,1), \mathbb{R})$ continuously. The Sobolev embedding theorem hence shows that

$$
H_{1 / 8} \subseteq L^{4}(\lambda ; \mathbb{R})
$$

continuously. This implies that for every $v \in H_{1 / 8}$ it holds that $v^{2} \in H$ and

$$
\sup _{w \in H_{1 / 8} \backslash\{0\}} \frac{\|w\|_{L^{4}(\lambda ; \mathbb{R})}}{\|w\|_{H_{1 / 8}}}<\infty .
$$

Item (iii) of Lemma 4.8 and the Cauchy-Schwarz inequality hence prove that 
(a) it holds for every $v, w \in H_{1 / 8}$ that $\left(v^{2}-w^{2}\right) \in H$ and

(b) there exists $C \in[1, \infty)$ such that for every $v, w \in H_{1 / 8}$ it holds that

$$
\begin{aligned}
& \left\|\bar{\partial}\left(v^{2}-w^{2}\right)\right\|_{H_{-1 / 2}} \leq C\left\|v^{2}-w^{2}\right\|_{H} \leq C\|v-w\|_{L^{4}(\lambda ; \mathbb{R})}\|v+w\|_{L^{4}(\lambda ; \mathbb{R})} \\
& \leq C^{3}\|v-w\|_{H_{1 / 8}}\|v+w\|_{H_{1 / 8}} \leq C^{3}\|v-w\|_{H_{1 / 8}}\left(1+\|v\|_{H_{1 / 8}}+\|w\|_{H_{1 / 8}}\right) .
\end{aligned}
$$

The proof of Lemma 4.17 is thus completed.

Corollary 4.18. Assume Setting 4.1. Then

(i) there exists a unique continuous function $\bar{F}: H_{1 / 8} \rightarrow H_{-1 / 2}$ which satisfies for every $v \in H_{1 / 2}$ that $\bar{F}(v)=F(v)$ and

(ii) there exists $C \in \mathbb{R}$ which satisfies for every $v, w \in H_{1 / 8}$ that

$$
\|\bar{F}(v)-\bar{F}(w)\|_{H_{-1 / 2}} \leq C\|v-w\|_{H_{1 / 8}}\left(1+\|v\|_{H_{1 / 8}}+\|w\|_{H_{1 / 8}}\right) .
$$

Proof of Corollary 4.18. Throughout this proof let $\bar{\partial}: H \rightarrow H_{-1 / 2}$ be the continuous function which satisfies for every $v \in W^{1,2}((0,1), \mathbb{R})$ that $\bar{\partial} v=\partial v$ (cf. item (ii) of Lemma 4.8). Note that item (ii) of Lemma 4.13 ensures that for every $v \in H_{1 / 2}$ it holds that $v^{2} \in W^{1,2}((0,1), \mathbb{R})$ and

$$
F(v)=\frac{c_{1}}{2} \partial\left(v^{2}\right)=\frac{c_{1}}{2} \bar{\partial}\left(v^{2}\right) .
$$

Lemma 4.17 (with $\bar{\partial}=\bar{\partial}$ in the notation of Lemma 4.17) hence shows that there exists $C \in \mathbb{R}$ such that for every $v, w \in H_{1 / 2}$ it holds that

$$
\begin{aligned}
\|F(v)-F(w)\|_{H_{-1 / 2}} & =\frac{\left|c_{1}\right|}{2}\left\|\partial\left(v^{2}\right)-\partial\left(w^{2}\right)\right\|_{H_{-1 / 2}}=\frac{\left|c_{1}\right|}{2}\left\|\bar{\partial}\left(v^{2}-w^{2}\right)\right\|_{H_{-1 / 2}} \\
& \leq C\|v-w\|_{H_{1 / 8}}\left(1+\|v\|_{H_{1 / 8}}+\|w\|_{H_{1 / 8}}\right) .
\end{aligned}
$$

Lemma 4.14 (with $X=H_{1 / 8}, d_{X}=\left(\left(H_{1 / 8} \times H_{1 / 8}\right) \ni\left(h_{1}, h_{2}\right) \mapsto\left\|h_{1}-h_{2}\right\|_{H_{1 / 8}} \in[0, \infty)\right), Y=H_{-1 / 2}$, $d_{Y}=\left(\left(H_{-1 / 2} \times H_{-1 / 2}\right) \ni\left(h_{1}, h_{2}\right) \mapsto\left\|h_{1}-h_{2}\right\|_{H_{-1 / 2}} \in[0, \infty)\right), S=H_{1 / 2}, F=F$ in the notation of Lemma 4.14) therefore establishes item (ii). Moreover, note that the fact that $H_{1 / 2} \subseteq H_{1 / 8}$ continuously and densely ensures that for every $v \in H_{1 / 8}$ there exist $\left(v_{n}\right)_{n \in \mathbb{N}} \subseteq H_{1 / 2}$ such that $\limsup _{n \rightarrow \infty}\left\|v-v_{n}\right\|_{H_{1 / 8}}=0$. This and item (ii) imply that for every $v, w \in H_{1 / 8}$ there exist $\left(v_{n}\right)_{n \in \mathbb{N}} \subseteq H_{1 / 2}$ and $\left(w_{n}\right)_{n \in \mathbb{N}} \subseteq H_{1 / 2}$ such that $\lim _{\sup _{n \rightarrow \infty}}\left(\left\|v-v_{n}\right\|_{H_{1 / 8}}+\left\|w-w_{n}\right\|_{H_{1 / 8}}\right)=0$ and

$$
\begin{aligned}
\|\bar{F}(v)-\bar{F}(w)\|_{H_{-1 / 2}} \leq & \lim \sup _{n \rightarrow \infty}\left\|\bar{F}(v)-\bar{F}\left(v_{n}\right)\right\|_{H_{-1 / 2}} \\
& +\limsup _{n \rightarrow \infty}\left\|\bar{F}\left(v_{n}\right)-\bar{F}\left(w_{n}\right)\right\|_{H_{-1 / 2}} \\
& +\limsup _{n \rightarrow \infty}\left\|\bar{F}\left(w_{n}\right)-\bar{F}(w)\right\|_{H_{-1 / 2}} \\
= & \lim \sup _{n \rightarrow \infty}\left\|\bar{F}\left(v_{n}\right)-\bar{F}\left(w_{n}\right)\right\|_{H_{-1 / 2}} \\
= & \lim \sup _{n \rightarrow \infty}\left\|F\left(v_{n}\right)-F\left(w_{n}\right)\right\|_{H_{-1 / 2}} .
\end{aligned}
$$

Combining this and (161) shows that there exists $C \in \mathbb{R}$ such that for every $v, w \in H_{1 / 8}$ there exist $\left(v_{n}\right)_{n \in \mathbb{N}} \subseteq H_{1 / 2}$ and $\left(w_{n}\right)_{n \in \mathbb{N}} \subseteq H_{1 / 2}$ such that

$$
\begin{aligned}
\|\bar{F}(v)-\bar{F}(w)\|_{H_{-1 / 2}} & \leq C \lim \sup _{n \rightarrow \infty}\left(\left\|v_{n}-w_{n}\right\|_{H_{1 / 8}}\left(1+\left\|v_{n}\right\|_{H_{1 / 8}}+\left\|w_{n}\right\|_{H_{1 / 8}}\right)\right) \\
& =C\|v-w\|_{H_{1 / 8}}\left(1+\|v\|_{H_{1 / 8}}+\|w\|_{H_{1 / 8}}\right) .
\end{aligned}
$$

This establishes item (iii). The proof of Corollary 4.18 is thus completed. 
Lemma 4.19. Assume Setting 4.1. Then

(i) it holds that $F \in \mathcal{C}^{1}\left(H_{1 / 2}, H\right)$ and

(ii) there exists $C \in(0, \infty)$ such that for every $\varepsilon \in(0, \infty), v, w \in H_{1 / 2}$ it holds that

$$
\left\langle F^{\prime}(v) w, w\right\rangle_{H} \leq \varepsilon\|v\|_{H_{1 / 2}}^{2}\|w\|_{H}^{2}+\frac{C}{\varepsilon^{2}}\|w\|_{H}^{2}+\|w\|_{H_{1 / 2}}^{2} .
$$

Proof of Lemma 4.19. Note that item (iii) of Lemma 4.13 establishes item (ii). Next observe that item (iii) of Lemma 4.3, Lemma 4.4, items (ii) and (iv) of Lemma 4.13, and the Cauchy-Schwarz inequality imply that for every $v, w \in H_{1 / 2}$ it holds that

$$
\begin{aligned}
& 2\left\langle F^{\prime}(v) w, w\right\rangle_{H}=2 c_{1}\langle w \partial v+v \partial w, w\rangle_{H}=2 c_{1}\langle w \partial v, w\rangle_{H}+2 c_{1}\langle v \partial w, w\rangle_{H} \\
& =2 c_{1}\left\langle\partial v, w^{2}\right\rangle_{H}+c_{1}\langle v, 2 w \partial w\rangle_{H}=2 c_{1}\left\langle\partial v, w^{2}\right\rangle_{H}+c_{1}\left\langle v, \partial\left(w^{2}\right)\right\rangle_{H} \\
& =2 c_{1}\left\langle\partial v, w^{2}\right\rangle_{H}-c_{1}\left\langle\partial v, w^{2}\right\rangle_{H}=c_{1}\left\langle\partial v, w^{2}\right\rangle_{H} \\
& \leq\left|c_{1}\right|\|\partial v\|_{H}\left\|w^{2}\right\|_{H}=\left|c_{1}\right|\|\partial v\|_{H}\|w\|_{L^{4}(\lambda ; \mathbb{R})}^{2}
\end{aligned}
$$

Moreover, note that Lemma 4.12 (with $q=2, p=4, r=2, \alpha=1 / 4$ in the notation of Lemma 4.12) and item (iii) of Lemma 4.3 prove that there exists $C \in \mathbb{R}$ such that for every $w \in H_{1 / 2} \subseteq$ $W_{0}^{1,2}((0,1), \mathbb{R})$ it holds that

$$
\|w\|_{L^{4}(\lambda ; \mathbb{R})} \leq C\|w\|_{W^{1,2}((0,1), \mathbb{R})}^{1 / 4}\|w\|_{H}^{3 / 4} .
$$

Items (iii) and (iv) of Lemma 4.3, (165), and the fact that for every $x_{1}, x_{2}, x_{3}, x_{4} \in \mathbb{R}$ it holds that $4 x_{1} x_{2} x_{3} x_{4} \leq\left|x_{1}\right|^{4}+\left|x_{2}\right|^{4}+\left|x_{3}\right|^{4}+\left|x_{4}\right|^{4}$ hence show that there exists $C \in(0, \infty)$ such that for every $\varepsilon \in(0, \infty), v, w \in H_{1 / 2}$ it holds that

$$
\begin{aligned}
& \left\langle F^{\prime}(v) w, w\right\rangle_{H} \leq C\|\partial v\|_{H}\|w\|_{W^{1,2}((0,1), \mathbb{R})}^{1 / 2}\|w\|_{H}^{3 / 2} \\
& \leq C\left|c_{0}\right|^{-1 / 2}\left[\sup _{u \in H_{1 / 2} \backslash\{0\}} \frac{\|u\|_{W^{1,2}((0,1), \mathbb{R})}}{\|u\|_{H_{1 / 2}}}\right]^{1 / 2}\|v\|_{H_{1 / 2}}\|w\|_{H_{1 / 2}}^{1 / 2}\|w\|_{H}^{3 / 2} \\
& =4\left[\left(\frac{\varepsilon}{2}\|v\|_{H_{1 / 2}}^{2}\|w\|_{H}^{2}\right)\left(\frac{\varepsilon}{2}\|v\|_{H_{1 / 2}}^{2}\|w\|_{H}^{2}\right)\left(\frac{C^{4}}{64 \varepsilon^{2}\left|c_{0}\right|^{2}}\left[\sup _{u \in H_{1 / 2} \backslash\{0\}} \frac{\|u\|_{W^{1,2}((0,1), \mathbb{R})}}{\|u\|_{H_{1 / 2}}}\right]^{2}\|w\|_{H}^{2}\right)\|w\|_{H_{1 / 2}}^{2}\right]^{\frac{1}{4}} \\
& \leq \frac{\varepsilon}{2}\|v\|_{H_{1 / 2}}^{2}\|w\|_{H}^{2}+\frac{\varepsilon}{2}\|v\|_{H_{1 / 2}}^{2}\|w\|_{H}^{2}+\frac{C^{4}}{64 \varepsilon^{2}\left|c_{0}\right|^{2}}\left[\sup _{u \in H_{1 / 2} \backslash\{0\}} \frac{\|u\|_{W^{1,2}((0,1), \mathbb{R})}}{\|u\|_{H_{1 / 2}}}\right]^{2}\|w\|_{H}^{2}+\|w\|_{H_{1 / 2}}^{2} \\
& =\varepsilon\|v\|_{H_{1 / 2}}^{2}\|w\|_{H}^{2}+\frac{C^{4}}{64 \varepsilon^{2}\left|c_{0}\right|^{2}}\left[\sup _{u \in H_{1 / 2} \backslash\{0\}} \frac{\|u\|_{W^{1,2}((0,1), \mathbb{R})}}{\|u\|_{H_{1 / 2}}}\right]^{2}\|w\|_{H}^{2}+\|w\|_{H_{1 / 2}}^{2}<\infty .
\end{aligned}
$$

This establishes item (iii). The proof of Lemma 4.19] is thus completed.

Lemma 4.20. Assume Setting [4.1, let $\alpha \in[0,1 / 2] \backslash\{1 / 4\}$, let $\mathcal{P}(\mathbb{H})$ be the power set of $\mathbb{H}$, let $\mathcal{P}_{0}(\mathbb{H})=\{\theta \in \mathcal{P}(\mathbb{H}): \theta$ is a finite set $\}$, and let $\left(P_{I}\right)_{I \in \mathcal{P}(\mathbb{H})} \subseteq L(H)$ satisfy for every $I \in \mathcal{P}(\mathbb{H})$, $v \in H$ that $P_{I}(v)=\sum_{h \in I}\langle h, v\rangle_{H} h$. Then it holds that

$$
\sup _{I \in \mathcal{P}_{0}(\mathbb{H I})} \sup _{v \in H_{\alpha+(1 / 2)} \backslash\{0\}} \frac{\left\|P_{I} F(v)\right\|_{H_{\alpha}}}{\|v\|_{H_{\alpha+(1 / 2)}}^{2}}<\infty .
$$


Proof of Lemma 4.20. Throughout this proof consider the notation in Triebel [38, Section 1.3.2 on page 24] (cf., e.g., Lunardi [30, Definition 1.2]). Note that item (iii) of Lemma 4.6 shows that for every $I \in \mathcal{P}_{0}(\mathbb{H}), v \in H_{\alpha+(1 / 2)}$ it holds that

$$
\left\|P_{I} F(v)\right\|_{H_{\alpha}} \leq\left(\sup _{u \in H_{\alpha} \backslash\{0\}} \frac{\|u\|_{H_{\alpha}}}{\|u\|_{W^{2 \alpha, 2}((0,1), \mathbb{R})}}\right)\left\|P_{I} F(v)\right\|_{W^{2 \alpha, 2}((0,1), \mathbb{R})}<\infty .
$$

Moreover, observe that the fact that

$$
\left(W^{2 \alpha+1,2}((0,1), \mathbb{R}) \ni v \mapsto \partial v \in W^{2 \alpha, 2}((0,1), \mathbb{R})\right) \in L\left(W^{2 \alpha+1,2}((0,1), \mathbb{R}), W^{2 \alpha, 2}((0,1), \mathbb{R})\right)
$$

(cf., e.g., Triebel [38, item (a) of Theorem 1 in Section 4.4 .2 on page 323, and Remark 2 in Section 4.4.2 on page 323]), item (ii) of Lemma 4.6. Lemma 4.7 (with $s=2 \alpha+1, q=2 \alpha+1$, $r=2 \alpha+1$ in the notation of Lemma 4.7), and item (ii) of Lemma 4.13 imply that there exists $C \in$ $(0, \infty)$ such that for every $v \in H_{\alpha+(1 / 2)}$ it holds that $v \in W^{2 \alpha+1,2}((0,1), \mathbb{R}), v^{2} \in W^{2 \alpha+1,2}((0,1), \mathbb{R})$, $F(v) \in W^{2 \alpha, 2}((0,1), \mathbb{R})$, and

$$
\begin{aligned}
& \|F(v)\|_{W^{2 \alpha, 2}((0,1), \mathbb{R})} \leq C\left\|v^{2}\right\|_{W^{2 \alpha+1,2}((0,1), \mathbb{R})} \\
& \leq C\left(\sup _{u \in W^{2 \alpha+1,2}((0,1), \mathbb{R}) \backslash\{0\}} \frac{\left\|u^{2}\right\|_{W^{2 \alpha+1,2}((0,1), \mathbb{R})}}{\|u\|_{W^{2 \alpha+1,2}((0,1), \mathbb{R})}^{2}}\right)\|v\|_{W^{2 \alpha+1,2}((0,1), \mathbb{R})}^{2}<\infty .
\end{aligned}
$$

Moreover, note that, e.g., Triebel [38, Definition 1 in Section 4.2.1 on page 310, Theorem 1 in Section 4.3 .1 on page 317, item (a) in Theorem 1 in Section 4.4 .2 on page 323, and Remark 2 in Section 4.4 .2 on page 324$]$ shows that for every $\iota \in(0,1 / 2)$ it holds that

$$
\left(H, W^{1,2}((0,1), \mathbb{R})\right)_{2 \iota, 2}=W^{2 \iota, 2}((0,1), \mathbb{R})
$$

and

$$
\sup _{x \in W^{2 \iota, 2}((0,1), \mathbb{R}) \backslash\{0\}}\left(\frac{\|x\|_{W^{2 \iota, 2}((0,1), \mathbb{R})}}{\|x\|_{\left(H, W^{1,2}((0,1), \mathbb{R})\right)_{2 \iota, 2}}}+\frac{\|x\|_{\left(H, W^{1,2}((0,1), \mathbb{R})\right)_{2 \iota, 2}}}{\|x\|_{W^{2 \iota, 2}((0,1), \mathbb{R})}}\right)<\infty .
$$

In addition, observe that the fact that $\mathbb{H} \subseteq W^{1,2}((0,1), \mathbb{R})$ is an orthogonal system ensures that for every $I \in \mathcal{P}_{0}(\mathbb{H}), v \in W^{1,2}((0,1), \mathbb{R})$ it holds that

$$
\left\|P_{I} v\right\|_{W^{1,2}((0,1), \mathbb{R})} \leq\|v\|_{W^{1,2}((0,1), \mathbb{R})} .
$$

The fact that for every $I \in \mathcal{P}_{0}(\mathbb{H}), v \in H$ it holds that $\left\|P_{I} v\right\|_{H} \leq\|v\|_{H}$, (172), (173), and, e.g., Lunardi [30, Theorem 1.6] therefore prove that for every $I \in \mathcal{P}_{0}(\mathbb{H}), v \in W^{2 \alpha, 2}((0,1), \mathbb{R})$ it holds that

$$
\left\|P_{I} v\right\|_{W^{2 \alpha, 2}((0,1), \mathbb{R})} \leq\|v\|_{W^{2 \alpha, 2}((0,1), \mathbb{R})} .
$$

Combining (169), (171), and item (ii) of Lemma 4.6 hence implies that there exists $C \in(0, \infty)$ such that for every $I \in \mathcal{P}_{0}(\mathbb{H}), v \in H_{\alpha+(1 / 2)}$ it holds that

$$
\begin{aligned}
\left\|P_{I} F(v)\right\|_{H_{\alpha}} \leq & C\left(\sup _{u \in H_{\alpha} \backslash\{0\}} \frac{\|u\|_{H_{\alpha}}}{\|u\|_{W^{2 \alpha, 2}((0,1), \mathbb{R})}}\right)\left(\sup _{u \in W^{2 \alpha+1,2}((0,1), \mathbb{R}) \backslash\{0\}} \frac{\left\|u^{2}\right\|_{W^{2 \alpha+1,2}((0,1), \mathbb{R})}}{\|u\|_{W^{2 \alpha+1,2}((0,1), \mathbb{R})}^{2}}\right) \\
& \cdot\left(\sup _{u \in H_{\alpha+(1 / 2)} \backslash\{0\}} \frac{\|u\|_{W^{2 \alpha+1,2}((0,1), \mathbb{R})}}{\|u\|_{H_{\alpha+(1 / 2)}}}\right)^{2}\|v\|_{H_{\alpha+(1 / 2)}}^{2}<\infty .
\end{aligned}
$$

The proof of Lemma 4.20 is thus completed. 
Lemma 4.21. Assume Setting 4.1. Then

(i) it holds for every $v \in H_{1 / 2}, \alpha \in(3 / 4, \infty)$ that

$$
\|F(v)\|_{H_{-\alpha}} \leq\left|c_{1}\right|\left|c_{0}\right|^{-\alpha}\left[\frac{1}{2} \sum_{n=1}^{\infty}|\pi n|^{2-4 \alpha}\right]^{1 / 2}\|v\|_{H}^{2}<\infty,
$$

(ii) it holds for every $v \in H_{1 / 2}, \alpha \in(1 / 4,1 / 2]$ that

$$
\begin{aligned}
\|F(v)\|_{H_{-\alpha}} \leq & \frac{\left|c_{1}\right|}{2}\left(\sup _{u \in H_{1 / 2} \backslash\{0\}} \frac{\left\|\partial\left(u^{2}\right)\right\|_{H_{-\alpha}}}{\left\|u^{2}\right\|_{W^{1-2 \alpha, 2}((0,1), \mathbb{R})}}\right)\left(\sup _{u \in H_{1 / 2} \backslash\{0\}} \frac{\left\|u^{2}\right\|_{W^{1-2 \alpha, 2}((0,1), \mathbb{R})}}{\|u\|_{W^{2(1-\alpha) / 3,2}((0,1), \mathbb{R})}^{2}}\right) \\
& \cdot\left(\sup _{u \in H_{1 / 2} \backslash\{0\}} \frac{\|u\|_{W^{2(1-\alpha) / 3,2}((0,1), \mathbb{R})}^{2}}{\|u\|_{H_{(1-\alpha) / 3}}^{2}}\right)\|v\|_{H_{(1-\alpha) / 3}}^{2}<\infty,
\end{aligned}
$$

and

(iii) it holds for every $v \in H_{1 / 2}$ that

$$
\|F(v)\|_{H} \leq \frac{\left|c_{1}\right|}{\sqrt{3} c_{0}}\|v\|_{H_{1 / 2}}^{2} .
$$

Proof of Lemma 4.21. Note that items (ii) and (iii) of Lemma 4.3 ensures that

$$
H_{1 / 2}=W_{0}^{1,2}((0,1), \mathbb{R}) \text {. }
$$

Next observe that item (ii) of Lemma 4.13 shows that for every $v \in H_{1 / 2}$ it holds that $v^{2} \in$ $W^{1,2}((0,1), \mathbb{R})$. This, (180), and Lemma 4.4 (with $u=u, v=v^{2}$ for $u, v \in H_{1 / 2}$ in the notation of Lemma 4.4) ensure that for every $u, v \in H_{1 / 2}$ it holds that $\left\langle\partial\left(v^{2}\right), u\right\rangle_{H}=-\left\langle v^{2}, \partial u\right\rangle_{H}$. Item (ii) of Lemma 4.13 and Lemma 4.10 (with $\alpha=\alpha-\frac{1}{2}$ for $\alpha \in\left(\frac{3}{4}, \infty\right)$ in the notation of Lemma 4.10) therefore prove that for every $v \in H_{1 / 2}, \alpha \in\left(\frac{3}{4}, \infty\right)$ it holds that

$$
\begin{aligned}
2\|F(v)\|_{H_{-\alpha}} & =\left\|c_{1} \partial\left(v^{2}\right)\right\|_{H_{-\alpha}}=\left|c_{1}\right| \sup _{u \in H_{\alpha} \backslash\{0\}} \frac{\left|\left\langle\partial\left(v^{2}\right), u\right\rangle_{H}\right|}{\|u\|_{H_{\alpha}}} \\
& =\left|c_{1}\right| \sup _{u \in H_{\alpha} \backslash\{0\}} \frac{\left|\left\langle v^{2}, \partial u\right\rangle_{H}\right|}{\|u\|_{H_{\alpha}}} \\
& \leq\left|c_{1}\right| \sup _{u \in H_{\alpha} \backslash\{0\}} \frac{\left\|v^{2}\right\|_{L^{1}(\lambda ; \mathbb{R})}\|\partial u\|_{L^{\infty}(\lambda ; \mathbb{R})}}{\|u\|_{H_{\alpha}}} \\
& \leq\left|c_{1}\right|\left|c_{0}\right|^{-\alpha}\|v\|_{H}^{2} \sqrt{2 \sum_{n=1}^{\infty}|\pi n|^{2-4 \alpha}}<\infty .
\end{aligned}
$$

This establishes item (ii). Next note that item (ii) of Lemma 4.13, Lemma 4.9] (with $\alpha=\alpha$ for $\alpha \in[0,1 / 2]$ in the notation of Lemma4.9), and Lemma4.7 (with $s=1-2 \alpha, q=2(1-\alpha) / 3, r=2(1-\alpha) / 3$ for $\alpha \in(1 / 4,1 / 2]$ in the notation of Lemma 4.7) show that for every $v \in H_{1 / 2}, \alpha \in(1 / 4,1 / 2]$ it holds that

$$
\begin{aligned}
& 2\|F(v)\|_{H_{-\alpha}}=\left|c_{1}\right|\left\|\partial\left(v^{2}\right)\right\|_{H_{-\alpha}} \leq\left|c_{1}\right|\left(\sup _{u \in H_{1 / 2} \backslash\{0\}} \frac{\left\|\partial\left(u^{2}\right)\right\|_{H_{-\alpha}}}{\left\|u^{2}\right\|_{W^{1-2 \alpha, 2}((0,1), \mathbb{R})}}\right)\left\|v^{2}\right\|_{W^{1-2 \alpha, 2}((0,1), \mathbb{R})} \\
& \leq\left|c_{1}\right|\left(\sup _{u \in H_{1 / 2} \backslash\{0\}} \frac{\left\|\partial\left(u^{2}\right)\right\|_{H_{-\alpha}}}{\left\|u^{2}\right\|_{W^{1-2 \alpha, 2}((0,1), \mathbb{R})}}\right)\left(\sup _{u \in H_{1 / 2} \backslash\{0\}} \frac{\left\|u^{2}\right\|_{W^{1-2 \alpha, 2}((0,1), \mathbb{R})}}{\|u\|_{W^{2(1-\alpha) / 3,2}((0,1), \mathbb{R})}^{2}}\right)\|v\|_{W^{2(1-\alpha) / 3,2}((0,1), \mathbb{R})}^{2}<\infty .
\end{aligned}
$$


Item (ii) of Lemma 4.6 hence implies item (iii). Furthermore, observe that items (iv) and (䜣) of Lemma 4.3 imply that for every $v \in H_{1 / 2}$ it holds that

$$
\|F(v)\|_{H}=\left|c_{1}\right|\|v \partial v\|_{H} \leq\left|c_{1}\right|\|v\|_{L^{\infty}(\lambda ; \mathbb{R})}\|\partial v\|_{H} \leq \frac{\left|c_{1}\right|}{\sqrt{3} c_{0}}\|v\|_{H_{1 / 2}}^{2} .
$$

This establishes item (iii). The proof of Lemma 4.21 is thus completed.

Corollary 4.22. Assume Setting [4.1] and let $\alpha_{1} \in(3 / 4, \infty), \alpha_{2} \in(1 / 4,1 / 2]$. Then

$$
\left[\sup _{v \in H_{1 / 2} \backslash\{0\}} \frac{\|F(v)\|_{H}}{\|v\|_{H_{1 / 2}}^{2}}\right]+\left[\sup _{v \in H_{1 / 2} \backslash\{0\}} \frac{\|F(v)\|_{H_{-\alpha_{2}}}}{\|v\|_{H_{\left(1-\alpha_{2}\right) / 3}}^{2}}\right]+\left[\sup _{v \in H_{1 / 2} \backslash\{0\}} \frac{\|F(v)\|_{H_{-\alpha_{1}}}}{\|v\|_{H}^{2}}\right]<\infty
$$

Proof of Corollary 4.22. Observe that item (ii) of Lemma 4.21 (with $\alpha=\alpha_{1}$ in the notation of item (ii) of Lemma 4.21) implies that

$$
\left[\sup _{v \in H_{1 / 2} \backslash\{0\}} \frac{\|F(v)\|_{H_{-\alpha}}}{\|v\|_{H}^{2}}\right]<\infty
$$

Next note that item (iii) of Lemma 4.21 (with $\alpha=\alpha_{2}$ in the notation of item (iii) of Lemma 4.21) shows that

$$
\left[\sup _{v \in H_{1 / 2} \backslash\{0\}} \frac{\|F(v)\|_{H_{-\alpha_{2}}}}{\|v\|_{H_{\left(1-\alpha_{2}\right) / 3}}^{2}}\right]<\infty
$$

Moreover, observe that item (iii) Lemma 4.21 ensures that

$$
\left[\sup _{v \in H_{1 / 2} \backslash\{0\}} \frac{\|F(v)\|_{H}}{\|v\|_{H_{1 / 2}}^{2}}\right]<\infty .
$$

Combining (185) and (186) therefore establishes (184). The proof of Corollary 4.22 is thus completed.

Lemma 4.23. Assume Setting 4.1. Then it holds for every $x \in H_{1 / 2}$ that $\langle x, F(x)\rangle_{H}=0$.

Proof of Lemma 4.23. Note that items (ii) and (ii) of Lemma 4.3, item (ii) of Lemma 4.13, and Lemma 4.4 (with $u=x, v=x^{2}$ for $x \in H_{1 / 2}$ in the notation of Lemma 4.4) ensure that for every $x \in H_{1 / 2}=W_{0}^{1,2}((0,1), \mathbb{R})$ it holds that $x^{2} \in W^{1,2}((0,1), \mathbb{R})$ and

$$
\begin{aligned}
2\langle x, F(x)\rangle_{H} & =2 c_{1}\langle x, x \partial x\rangle_{H}=c_{1}\left\langle x, \partial\left(x^{2}\right)\right\rangle_{H} \\
& =-c_{1}\left\langle\partial x, x^{2}\right\rangle_{H}=-c_{1}\langle x \partial x, x\rangle_{H}=-\langle F(x), x\rangle_{H} .
\end{aligned}
$$

The proof of Lemma 4.23 is thus completed.

Corollary 4.24. Assume Setting 4.1 and let $\iota \in(1 / 4, \infty), v \in H_{1 / 2}, w \in H_{\max \{1 / 2, \iota\}}$. Then it holds that

$$
\begin{aligned}
& \langle v, F(v+w)\rangle_{H} \\
& \leq \frac{3\left|c_{1}\right|^{2}}{8\left|c_{0}\right|}\left[\sup _{u \in H_{\iota} \backslash\{0\}} \frac{\|u\|_{L^{\infty}(\lambda ; \mathbb{R})}}{\|u\|_{H_{\iota}}}+\sup _{u \in H_{\iota} \backslash\{0\}} \frac{\|u\|_{L^{4}(\lambda ; \mathbb{R})}^{2}}{\|u\|_{H_{\iota}}^{2}}\right]^{2}\left(\|v\|_{H}^{2}+\|w\|_{H_{\iota}}^{2}\right)\|w\|_{H_{\iota}}^{2}+\|v\|_{H_{1 / 2}}^{2}<\infty .
\end{aligned}
$$


Proof of Corollary 4.24. Throughout this proof assume w.l.o.g. that $c_{1} \neq 0$ and let $C \in[0, \infty]$ satisfy that

$$
C=\sup _{u \in H_{\iota} \backslash\{0\}} \frac{\|u\|_{L}{ }_{(\lambda ; \mathbb{R})}}{\|u\|_{H_{\iota}}}+\sup _{u \in H_{\iota} \backslash\{0\}} \frac{\|u\|_{L^{4}(\lambda ; \mathbb{R})}^{2}}{\|u\|_{H_{\iota}}^{2}} .
$$

Note that the Sobolev embedding theorem and item (ii) of Lemma 4.6 ensure that $C \in(0, \infty)$. Next observe that Lemma 4.23, item (ii) of Lemma 4.13, and Lemma 4.4 (with $u=v, v=w^{2}$ in the notation of Lemma 4.4) ensure that

$$
\begin{aligned}
\langle v, F(v+w)\rangle_{H} & =c_{1}\langle v,(v+w)(\partial v+\partial w)\rangle_{H} \\
& =c_{1}\langle v, v \partial v\rangle_{H}+c_{1}\langle v, w \partial v\rangle_{H}+c_{1}\langle v, v \partial w\rangle_{H}+c_{1}\langle v, w \partial w\rangle_{H} \\
& =c_{1}\langle v, w \partial v\rangle_{H}+c_{1}\langle v, v \partial w\rangle_{H}+\frac{c_{1}}{2}\left\langle v, \partial\left(w^{2}\right)\right\rangle_{H} \\
& =c_{1}\langle v, w \partial v\rangle_{H}+c_{1}\left\langle v^{2}, \partial w\right\rangle_{H}-\frac{c_{1}}{2}\left\langle\partial v, w^{2}\right\rangle_{H} .
\end{aligned}
$$

Lemma 4.4 (with $u=w, v=v^{2}$ in the notation of Lemma 4.4) and item (ii) of Lemma 4.13 therefore imply that

$$
\begin{aligned}
\langle v, F(v+w)\rangle_{H} & =c_{1}\langle v \partial v, w\rangle_{H}-2 c_{1}\langle v \partial v, w\rangle_{H}-\frac{c_{1}}{2}\left\langle\partial v, w^{2}\right\rangle_{H} \\
& =-c_{1}\langle v \partial v, w\rangle_{H}-\frac{c_{1}}{2}\left\langle\partial v, w^{2}\right\rangle_{H} \\
& \leq\left|c_{1}\right|\left|\langle v \partial v, w\rangle_{H}\right|+\frac{\left|c_{1}\right|}{2}\left|\left\langle\partial v, w^{2}\right\rangle_{H}\right|
\end{aligned}
$$

Hölder's inequality and item (iv) of Lemma 4.3 hence prove that

$$
\begin{aligned}
&\langle v, F(v+w)\rangle_{H} \leq \frac{\left|c_{1}\right|}{2}\left(2\|v\|_{H}\|\partial v\|_{H}\|w\|_{L^{\infty}(\lambda ; \mathbb{R})}+\|\partial v\|_{H}\|w\|_{L^{4}(\lambda ; \mathbb{R})}^{2}\right) \\
& \leq \frac{\left|c_{1}\right|}{2\left|c_{0}\right|^{1 / 2}}\left(2\|v\|_{H}\|v\|_{H_{1 / 2}}\|w\|_{L^{\infty}(\lambda ; \mathbb{R})}+\|v\|_{H_{1 / 2}}\|w\|_{L^{4}(\lambda ; \mathbb{R})}^{2}\right) \\
& \leq \frac{\left|c_{1}\right|}{2\left|c_{0}\right|^{1 / 2}}\left(2\|v\|_{H}\|v\|_{H_{1 / 2}}\left[\sup _{u \in H_{\iota} \backslash\{0\}} \frac{\|u\|_{L^{\infty}(\lambda ; \mathbb{R})}}{\|u\|_{H_{\iota}}}\right]\|w\|_{H_{\iota}}\right. \\
&\left.\quad+\|v\|_{H_{1 / 2}}\left[\sup _{u \in H_{\iota} \backslash\{0\}} \frac{\|u\|_{L^{4}(\lambda ; \mathbb{R})}}{\|u\|_{H_{\iota}}}\right]^{2}\|w\|_{H_{\iota}}^{2}\right) \\
& \leq \frac{\left|c_{1}\right| C}{2\left|c_{0}\right|^{1 / 2}}\left(2\|v\|_{H}\|v\|_{H_{1 / 2}}\|w\|_{H_{\iota}}+\|v\|_{H_{1 / 2}}\|w\|_{H_{\iota}}^{2}\right) .
\end{aligned}
$$

The fact that for every $x, y \in \mathbb{R}, \varepsilon \in(0, \infty)$ it holds that $2 x y \leq \frac{x^{2}}{\varepsilon}+\varepsilon y^{2}$ therefore shows that

$$
\begin{aligned}
& \langle v, F(v+w)\rangle_{H} \\
& \leq \frac{\left|c_{1}\right| C}{2\left|c_{0}\right|^{1 / 2}}\left(\left[\frac{3\left|c_{1}\right| C}{4\left|c_{0}\right|^{1 / 2}}\|v\|_{H}^{2}\|w\|_{H_{\iota}}^{2}+\frac{4\left|c_{0}\right|^{1 / 2}}{3\left|c_{1}\right| C}\|v\|_{H_{1 / 2}}^{2}\right]+\frac{1}{2}\left[\frac{4\left|c_{0}\right|^{1 / 2}}{3\left|c_{1}\right| C}\|v\|_{H_{1 / 2}}^{2}+\frac{3\left|c_{1}\right| C}{4\left|c_{0}\right|^{1 / 2}}\|w\|_{H_{\iota}}^{4}\right]\right) \\
& =\frac{3\left|c_{1}\right|^{2} C^{2}}{8\left|c_{0}\right|}\|v\|_{H}^{2}\|w\|_{H_{\iota}}^{2}+\frac{2}{3}\|v\|_{H_{1 / 2}}^{2}+\frac{1}{3}\|v\|_{H_{1 / 2}}^{2}+\frac{3\left|c_{1}\right|^{2} C^{2}}{16\left|c_{0}\right|}\|w\|_{H_{\iota}}^{4} \\
& =\frac{3\left|c_{1}\right|^{2} C^{2}}{8\left|c_{0}\right|}\|v\|_{H}^{2}\|w\|_{H_{\iota}}^{2}+\frac{3\left|c_{1}\right|^{2} C^{2}}{16 c_{0} \mid}\|w\|_{H_{\iota}}^{4}+\|v\|_{H_{1 / 2}}^{2} \\
& \leq \frac{3\left|c_{1}\right|^{2} C^{2}}{8\left|c_{0}\right|}\left(\|v\|_{H}^{2}+\|w\|_{H_{\iota}}^{2}\right)\|w\|_{H_{\iota}}^{2}+\|v\|_{H_{1 / 2}}^{2} .
\end{aligned}
$$

The proof of Corollary 4.24 is thus completed. 


\section{$5 \quad$ Existence and uniqueness of mild solutions to stochastic Burgers equations}

In this section we prove in Theorem 5.10 below the unique existence of suitably regular mild solutions to stochastic Burgers equations with additive trace class noise. To do so, we first establish in Lemmas 5.15.6 (cf., e.g., Blömker \& Jentzen [4, Lemma 5.5]), Lemma 5.7 (cf., e.g., Kloeden \& Neuenkirch [27, Lemma 2.1]), and Lemma 5.8 (cf., e.g., Blömker \& Jentzen [4, Lemma 4.3]) a few elementary and partially well-known auxiliary results. Only for the sake of completeness we include in this section also a proof of Lemma 5.7. Thereafter, we combine these auxiliary results with the results from Subsection 4.2 and the abstract existence and uniqueness result in Blömker \& Jentzen [4, Theorem 3.1] to establish in Theorem 5.10 below the main result of this article.

Lemma 5.1. Assume Setting 4.1, let $T \in(0, \infty), \iota \in(1 / 4, \infty), \xi \in H$, let $I \subseteq \mathbb{H}$ be a finite set, let $P \in L(H)$ satisfy for every $v \in H$ that $P v=\sum_{h \in I}\langle h, v\rangle_{H} h$, and let $O, X \in \mathcal{C}([0, T], P(H))$ satisfy for every $t \in[0, T]$ that

$$
X_{t}=e^{t A} P \xi+\int_{0}^{t} e^{(t-s) A} P F\left(X_{s}\right) d s+O_{t} .
$$

Then it holds for every $t \in[0, T]$ that

$$
\begin{aligned}
& \left\|X_{t}\right\|_{H} \leq\left\|O_{t}\right\|_{H} \\
& +\left(\|\xi\|_{H}^{2}+\frac{3\left|c_{1}\right|^{2}}{8\left|c_{0}\right|}\left[\sup _{u \in H_{\iota} \backslash\{0\}} \frac{\|u\|_{L^{\infty}(\lambda ; \mathbb{R})}}{\|u\|_{H_{\iota}}}+\sup _{u \in H_{\iota} \backslash\{0\}} \frac{\|u\|_{L^{4}(\lambda ; \mathbb{R})}^{2}}{\|u\|_{H_{\iota}}^{2}}\right]^{2}\left[1+\sup _{u \in[0, T]}\left\|O_{u}\right\|_{H_{\iota}}^{2}\right]^{2} T\right)^{\frac{1}{2}} \\
& \quad \cdot \exp \left(\frac{3\left|c_{1}\right|^{2}}{16\left|c_{0}\right|}\left[\sup _{u \in H_{\iota} \backslash\{0\}} \frac{\|u\|_{L^{\infty}(\lambda ; \mathbb{R})}}{\|u\|_{H_{\iota}}}+\sup _{u \in H_{\iota} \backslash\{0\}} \frac{\|u\|_{L^{4}(\lambda ; \mathbb{R})}^{2}}{\|u\|_{H_{\iota}}^{2}}\right]^{2}\left[1+\sup _{u \in[0, T]}\left\|O_{u}\right\|_{H_{\iota}}^{2}\right]^{2} T\right)<\infty .
\end{aligned}
$$

Proof of Lemma 5.1. Throughout this proof let $C \in[0, \infty]$ satisfy that

$$
C=\frac{3\left|c_{1}\right|^{2}}{8\left|c_{0}\right|}\left[\sup _{u \in H_{\iota} \backslash\{0\}} \frac{\|u\|_{L^{\infty}(\lambda ; \mathbb{R})}}{\|u\|_{H_{\iota}}}+\sup _{u \in H_{\iota} \backslash\{0\}} \frac{\|u\|_{L^{4}(\lambda ; \mathbb{R})}^{2}}{\|u\|_{H_{\iota}}^{2}}\right]^{2}
$$

and let $Z:[0, T] \rightarrow P(H)$ be the function which satisfies for every $t \in[0, T]$ that $Z_{t}=X_{t}-O_{t}$. Observe that the Sobolev embedding theorem and item (ii) of Lemma 4.6 ensure that $C \in[0, \infty$ ). Next note that for every $t \in[0, T]$ it holds that

$$
Z_{t}=e^{t A} P \xi+\int_{0}^{t} e^{(t-s) A} P F\left(Z_{s}+O_{s}\right) d s .
$$

This implies for every $t \in[0, T]$ that

$$
Z_{t}=P \xi+\int_{0}^{t}\left[A Z_{s}+P F\left(Z_{s}+O_{s}\right)\right] d s .
$$

Therefore, we obtain that for every $t \in[0, T]$ it holds that

$$
\begin{aligned}
\left\|Z_{t}\right\|_{H}^{2} & =\|P \xi\|_{H}^{2}+2 \int_{0}^{t}\left\langle Z_{s}, A Z_{s}+P F\left(Z_{s}+O_{s}\right)\right\rangle_{H} d s \\
& \leq\|\xi\|_{H}^{2}+2 \int_{0}^{t}\left\langle Z_{s}, A Z_{s}+F\left(Z_{s}+O_{s}\right)\right\rangle_{H} d s .
\end{aligned}
$$


Corollary 4.24 (with $\iota=\iota, v=Z_{s}, w=O_{s}$ for $s \in[0, T]$ in the notation of Corollary 4.24) hence proves that for every $t \in[0, T]$ it holds that

$$
\begin{aligned}
\left\|Z_{t}\right\|_{H}^{2} & \leq\|\xi\|_{H}^{2}+2 \int_{0}^{t} \frac{C}{2}\left[\left\|Z_{s}\right\|_{H}^{2}+\left\|O_{s}\right\|_{H_{\iota}}^{2}\right]\left\|O_{s}\right\|_{H_{\iota}}^{2} d s \\
& \leq\|\xi\|_{H}^{2}+C\left[1+\sup _{u \in[0, T]}\left\|O_{u}\right\|_{H_{\iota}}^{2}\right]^{2} \int_{0}^{t}\left[1+\left\|Z_{s}\right\|_{H}^{2}\right] d s .
\end{aligned}
$$

The fact that $O, Z \in \mathcal{C}([0, T], P(H))$ and Gronwall's lemma therefore establish that for every $t \in[0, T]$ it holds that

$$
\left\|Z_{t}\right\|_{H}^{2} \leq\left(\|\xi\|_{H}^{2}+C\left[1+\sup _{u \in[0, T]}\left\|O_{u}\right\|_{H_{\iota}}^{2}\right]^{2} T\right) \exp \left(C\left[1+\sup _{u \in[0, T]}\left\|O_{u}\right\|_{H_{\iota}}^{2}\right]^{2} T\right) .
$$

This completes the proof of Lemma 5.1.

Lemma 5.2. Assume Setting 1.2, let $\alpha \in \mathbb{R}, I \subseteq \mathbb{H}$, and let $R: H_{\max \{\alpha, 0\}} \rightarrow H_{\alpha}$ be the function which satisfies for every $v \in H_{\max \{\alpha, 0\}}$ that $R v=\sum_{h \in I}\langle h, v\rangle_{H} h$. Then

(i) it holds that there exists $P \in L\left(H_{\alpha}\right)$ which satisfies for every $v \in H_{\max \{\alpha, 0\}}$ that $P v=R v$ and

(ii) it holds that $\|P\|_{L\left(H_{\alpha}\right)} \leq 1$.

Proof of Lemma 5.2. Note that for every $v \in H_{\max \{\alpha, 0\}}$ it holds that

$$
\|R v\|_{H_{\max \{\alpha, 0\}}}^{2}=\sum_{h \in I}\left|\langle h, v\rangle_{H}\right|^{2}\left|\mathfrak{v}_{h}\right|^{2 \max \{\alpha, 0\}} \leq \sum_{h \in \mathbb{H}}\left|\langle h, v\rangle_{H}\right|^{2}\left|\mathfrak{v}_{h}\right|^{2 \max \{\alpha, 0\}}=\|v\|_{H_{\max \{\alpha, 0\}}}^{2} .
$$

Furthermore, observe that the fact that $\forall v \in H_{\max \{\alpha, 0\}}: R v \in H$ ensures that for every $v \in$ $H_{\max \{\alpha, 0\}}$ it holds that

$$
\begin{aligned}
& \|R v\|_{H_{\min \{\alpha, 0\}}}^{2}=\left\|(-A)^{\min \{\alpha, 0\}} R v\right\|_{H}^{2}=\left\|\sum_{h \in \mathbb{H}}\left|\mathfrak{v}_{h}\right|^{\min \{\alpha, 0\}}\langle h, R v\rangle_{H} h\right\|_{H}^{2} \\
& =\sum_{h \in I}\left|\langle h, v\rangle_{H}\right|^{2}\left|\mathfrak{v}_{h}\right|^{2 \min \{\alpha, 0\}} \leq \sum_{h \in \mathbb{H}}\left|\langle h, v\rangle_{H}\right|^{2}\left|\mathfrak{v}_{h}\right|^{2 \min \{\alpha, 0\}}=\|v\|_{H_{\min \{\alpha, 0\}}}^{2}
\end{aligned}
$$

Combining this and (203) proves that for every $v \in H_{\max \{\alpha, 0\}}$ it holds that

$$
\|R v\|_{H_{\alpha}} \leq\|v\|_{H_{\alpha}} .
$$

The fact that $H_{\max \{\alpha, 0\}} \subseteq H_{\alpha}$ densely therefore establishes items (ii) and (iii). The proof of Lemma 5.2 is thus completed.

Lemma 5.3. Assume Setting 4.1, let $\mathcal{P}(\mathbb{H})$ be the power set of $\mathbb{H}$, let $T \in(0, \infty), \iota \in[0,1)$, $\gamma \in(1 / 4, \infty), \xi \in H_{\iota}, \mathcal{P}_{0}(\mathbb{H})=\{\theta \in \mathcal{P}(\mathbb{H}): \theta$ is a finite set $\}$, let $\left(P_{I}\right)_{I \in \mathcal{P}(\mathbb{H})} \subseteq L(H)$ satisfy for every $I \in \mathcal{P}(\mathbb{H}), v \in H$ that $P_{I}(v)=\sum_{h \in I}\langle h, v\rangle_{H} h$, let $O^{I} \in \mathcal{C}\left([0, T], P_{I}(H)\right), I \in \mathcal{P}_{0}(\mathbb{H})$, satisfy $\sup _{I \in \mathcal{P}_{0}(\mathbb{H})} \sup _{u \in[0, T]}\left\|O_{u}^{I}\right\|_{H_{\max \{\gamma, \iota\}}}<\infty$, let $X^{I} \in \mathcal{C}\left([0, T], P_{I}(H)\right), I \in \mathcal{P}_{0}(\mathbb{H})$, and assume for every $I \in \mathcal{P}_{0}(\mathbb{H}), t \in[0, T]$ that

$$
X_{t}^{I}=e^{t A} P_{I} \xi+\int_{0}^{t} e^{(t-s) A} P_{I} F\left(X_{s}^{I}\right) d s+O_{t}^{I} .
$$

Then it holds that

$$
\sup _{I \in \mathcal{P}_{0}(\mathbb{H})} \sup _{t \in[0, T]}\left\|X_{t}^{I}\right\|_{H_{\iota}}<\infty
$$


Proof of Lemma 5.3. Note that Corollary 4.22 (with $\alpha_{1}=\alpha_{1}, \alpha_{2}=\alpha_{2}$ for $\alpha_{1} \in(3 / 4, \infty), \alpha_{2} \in$ $(1 / 4,1 / 2]$ in the notation of Corollary 4.22) shows that for every $\alpha_{1} \in(3 / 4, \infty), \alpha_{2} \in(1 / 4,1 / 2]$ it holds that

$$
\left(\sup _{v \in H_{1 / 2} \backslash\{0\}} \frac{\|F(v)\|_{H}}{\|v\|_{H_{1 / 2}}^{2}}\right)+\left(\sup _{v \in H_{1 / 2} \backslash\{0\}} \frac{\|F(v)\|_{H_{-\alpha_{2}}}}{\|v\|_{H_{\left(1-\alpha_{2}\right) / 3}}^{2}}\right)+\left(\sup _{v \in H_{1 / 2} \backslash\{0\}} \frac{\|F(v)\|_{H_{-\alpha_{1}}}}{\|v\|_{H}^{2}}\right)<\infty .
$$

In addition, observe that Lemma 5.2 (with $\alpha=-\alpha, I=I, R=\left(H \ni x \mapsto P_{I} x \in H_{-\alpha}\right.$ ) for $I \in \mathcal{P}_{0}(\mathbb{H}), \alpha \in \mathbb{R}$ in the notation of Lemma [5.2) proves that for every $x \in H, I \in \mathcal{P}_{0}(\mathbb{H}), \alpha \in \mathbb{R}$ it holds that

$$
\left\|P_{I} x\right\|_{H_{-\alpha}} \leq\left\|P_{I}\right\|_{L_{\left(H_{-\alpha}\right)}}\|x\|_{H_{-\alpha}} \leq\|x\|_{H_{-\alpha}}
$$

Combining this and (208) ensures that for every $\alpha_{1} \in(3 / 4, \infty), \alpha_{2} \in(1 / 4,1 / 2]$ it holds that

$$
\begin{aligned}
& \sup _{I \in \mathcal{P}_{0}(\mathbb{H})}\left(\sup _{v \in H_{1 / 2} \backslash\{0\}} \frac{\left\|P_{I} F(v)\right\|_{H}}{\|v\|_{H_{1 / 2}}^{2}}\right)<\infty, \\
& \sup _{I \in \mathcal{P}_{0}(\mathbb{H})}\left(\sup _{v \in H_{1 / 2} \backslash\{0\}} \frac{\left\|P_{I} F(v)\right\|_{H_{-\alpha_{2}}}}{\|v\|_{H_{\left(1-\alpha_{2}\right) / 3}}^{2}}\right)<\infty,
\end{aligned}
$$

and

$$
\sup _{I \in \mathcal{P}_{0}(\mathbb{H})}\left(\sup _{v \in H_{1 / 2} \backslash\{0\}} \frac{\left\|P_{I} F(v)\right\|_{H_{-\alpha_{1}}}}{\|v\|_{H}^{2}}\right)<\infty .
$$

Moreover, observe that Lemma 5.1 (with $T=T, \iota=\max \{\gamma, \iota\}, \xi=\xi, I=I, P=P_{I}, O=O^{I}$, $X=X^{I}$ for $I \in \mathcal{P}_{0}(\mathbb{H})$ in the notation of Lemma 5.1) implies that

$$
\sup _{I \in \mathcal{P}_{0}(\mathbb{H})} \sup _{t \in[0, T]}\left\|X_{t}^{I}\right\|_{H}<\infty .
$$

Combining (212) and Lemma 3.2 (with $(\Omega, \mathcal{F}, \mathbb{P})=\left(\{1\},\{\emptyset,\{1\}\},\left(\{\emptyset,\{1\}\} \ni A \mapsto \mathbb{1}_{A}(1) \in\right.\right.$ $[0,1])), T=T, \beta=1 / 2, \gamma=1 / 2, \xi=\left(\{1\} \ni \omega \mapsto P_{I} \xi \in H_{1 / 2}\right), F=\left(H_{1 / 2} \ni v \mapsto P_{I} F(v) \in H\right)$, $\kappa=([0, T] \ni t \mapsto t \in[0, T]), Z=\left([0, T] \times\{1\} \ni(t, \omega) \mapsto X_{t}^{I} \in H_{1 / 2}\right), O=([0, T] \times\{1\} \ni(t, \omega) \mapsto$ $\left.O_{t}^{I} \in H_{1 / 2}\right), Y=\left([0, T] \times\{1\} \ni(t, \omega) \mapsto X_{t}^{I} \in H\right), p=1, \rho=\rho, \alpha=\alpha_{1}$ for $\alpha_{1} \in(3 / 4,1-\rho)$, $\rho \in[0,1 / 4), I \in \mathcal{P}_{0}(\mathbb{H})$ in the notation of Lemma [3.2) hence shows that for every $\rho \in[0,1 / 4)$, $\alpha_{1} \in(3 / 4,1-\rho), I \in \mathcal{P}_{0}(\mathbb{H}), t \in[0, T]$ it holds that

$$
\left\|X_{t}^{I}\right\|_{H_{\rho}} \leq\left\|P_{I} \xi\right\|_{H_{\rho}}+\left\|O_{t}^{I}\right\|_{H_{\rho}}+\frac{T^{1-\alpha_{1}-\rho}}{1-\alpha_{1}-\rho}\left(\sup _{v \in H_{1 / 2}} \frac{\left\|P_{I} F(v)\right\|_{H_{-\alpha}}}{1+\|v\|_{H}^{2}}\right)\left(1+\sup _{u \in[0, T]}\left\|X_{u}^{I}\right\|_{H}^{2}\right) .
$$

This, (212), (213), and the assumption that $\sup _{I \in \mathcal{P}_{0}(\mathbb{H})} \sup _{u \in[0, T]}\left\|O_{u}^{I}\right\|_{H_{\iota}}<\infty$ show that for every $\rho \in[0,1 / 4)$ with $\rho \leq \iota$ it holds that

$$
\sup _{I \in \mathcal{P}_{0}(\mathbb{H})} \sup _{t \in[0, T]}\left\|X_{t}^{I}\right\|_{H_{\rho}}<\infty .
$$

Furthermore, observe that Lemma 3.3 (with $H=H,(\Omega, \mathcal{F}, \mathbb{P})=(\{1\},\{\emptyset,\{1\}\},(\{\emptyset,\{1\}\} \ni$ $\left.\left.A \mapsto \mathbb{1}_{A}(1) \in[0,1]\right)\right), T=T, \beta=1 / 2, \gamma=1 / 2, \xi=\left(\{1\} \ni \omega \mapsto P_{I} \xi \in H_{1 / 2}\right), F=\left(H_{1 / 2} \ni\right.$ $\left.v \mapsto P_{I} F(v) \in H\right), \kappa=([0, T] \ni t \mapsto t \in[0, T]), Z=\left([0, T] \times\{1\} \ni(t, \omega) \mapsto X_{t}^{I} \in H_{1 / 2}\right)$, $O=\left([0, T] \times\{1\} \ni(t, \omega) \mapsto O_{t}^{I} \in H_{1 / 2}\right), Y=\left([0, T] \times\{1\} \ni(t, \omega) \mapsto X_{t}^{I} \in H\right), p=1, \rho=\left(1-\alpha_{2}\right) / 3$, $\eta=\eta, \alpha_{1}=\alpha_{1}, \alpha_{2}=\alpha_{2}$ for $\alpha_{1} \in\left(3 / 4,\left(2+\alpha_{2}\right) / 3\right), \alpha_{2} \in(1 / 4,1 / 2), \eta \in[1 / 4,1 / 2], I \in \mathcal{P}_{0}(\mathbb{H})$ in the 
notation of Lemma 3.3), (211), and (212) ensure that for every $\alpha_{2} \in(1 / 4,1 / 2), \alpha_{1} \in\left(3 / 4,\left(2+\alpha_{2}\right) / 3\right)$, $\eta \in[1 / 4,1 / 2], I \in \mathcal{P}_{0}(\mathbb{H}), t \in[0, T]$ it holds that

$$
\begin{aligned}
\left\|X_{t}^{I}\right\|_{H_{\eta}} \leq & \left\|P_{I} \xi\right\|_{H_{\eta}}+\left\|O_{t}^{I}\right\|_{H_{\eta}}+\frac{T^{1-\alpha_{2}-\eta}}{1-\alpha_{2}-\eta}\left(\sup _{v \in H_{1 / 2}} \frac{\left\|P_{I} F(v)\right\|_{H_{-\alpha}}}{1+\|v\|_{H_{\left(1-\alpha_{2}\right) / 3}}^{2}}\right) \\
\cdot & {\left[1+\|\xi\|_{H_{\left(1-\alpha_{2}\right) / 3}}+\sup _{u \in[0, T]}\left\|O_{u}^{I}\right\|_{H_{\left(1-\alpha_{2}\right) / 3}}\right.} \\
& \left.\quad+\frac{T^{1-\alpha_{1}-\left(\left(1-\alpha_{2}\right) / 3\right)}}{1-\alpha_{1}-\left(\left(1-\alpha_{2}\right) / 3\right)}\left(\sup _{v \in H_{1 / 2}} \frac{\left\|P_{I} F(v)\right\|_{H_{-\alpha}}}{1+\|v\|_{H}^{2}}\right)\left(1+\sup _{u \in[0, T]}\left\|X_{u}^{I}\right\|_{H}^{2}\right)\right]^{2} .
\end{aligned}
$$

Combining (211) $-(213)$ and the assumption that $\sup _{I \in \mathcal{P}_{0}(\mathbb{H I})} \sup _{u \in[0, T]}\left\|O_{u}^{I}\right\|_{H_{\iota}}<\infty$ hence implies that for every $\eta \in[1 / 4,1 / 2]$ with $\eta \leq \iota$ it holds that

$$
\sup _{I \in \mathcal{P}_{0}(\mathbb{H})} \sup _{t \in[0, T]}\left\|X_{t}^{I}\right\|_{H_{\eta}}<\infty .
$$

Moreover, note that Lemma 3.4 (with $H=H,(\Omega, \mathcal{F}, \mathbb{P})=\left(\{1\},\{\emptyset,\{1\}\},\left(\{\emptyset,\{1\}\} \ni A \mapsto \mathbb{1}_{A}(1) \in\right.\right.$ $[0,1])), T=T, \beta=\kappa, \gamma=1 / 2, \xi=\left(\{1\} \ni \omega \mapsto P_{I} \xi \in H_{\kappa}\right), F=\left(H_{1 / 2} \ni v \mapsto P_{I} F(v) \in H\right)$, $\kappa=([0, T] \ni t \mapsto t \in[0, T]), Z=\left([0, T] \times\{1\} \ni(t, \omega) \mapsto X_{t}^{I} \in H_{1 / 2}\right), O=([0, T] \times\{1\} \ni(t, \omega) \mapsto$ $\left.O_{t}^{I} \in H_{\kappa}\right), Y=\left([0, T] \times\{1\} \ni(t, \omega) \mapsto X_{t}^{I} \in H\right), p=1, \rho=\left(1-\alpha_{2}\right) / 3, \eta=1 / 2, \iota=\kappa, \alpha_{1}=\alpha_{1}$, $\alpha_{2}=\alpha_{2}$ for $\alpha_{1} \in\left[0,\left(2+\alpha_{2}\right) / 3\right), \alpha_{2} \in[0,1 / 2), \kappa \in[1 / 2,1), I \in \mathcal{P}_{0}(\mathbb{H})$ in the notation of Lemma 3.4) and (210) -(212) prove that for every $\alpha_{2} \in(1 / 4,1 / 2), \alpha_{1} \in\left(3 / 4,\left(2+\alpha_{2}\right) / 3\right), \kappa \in[1 / 2,1), I \in \mathcal{P}_{0}(\mathbb{H})$, $t \in[0, T]$ it holds that

$$
\begin{aligned}
\left\|X_{t}^{I}\right\|_{H_{\kappa}} \leq & \left\|P_{I} \xi\right\|_{H_{\kappa}}+\sup _{u \in[0, T]}\left\|O_{u}^{I}\right\|_{H_{\kappa}}+\frac{T^{1-\kappa}}{1-\kappa}\left(\sup _{v \in H_{1 / 2}} \frac{\left\|P_{I} F(v)\right\|_{H}}{1+\|v\|_{H_{1 / 2}}^{2}}\right) \\
& \cdot\left[1+\|\xi\|_{H_{1 / 2}}+\sup _{u \in[0, T]}\left\|O_{u}^{I}\right\|_{H_{1 / 2}}+\frac{T^{(1 / 2)-\alpha_{2}}}{(1 / 2)-\alpha_{2}}\left(\sup _{v \in H_{1 / 2}} \frac{\left\|P_{I} F(v)\right\|_{H_{-\alpha_{2}}}}{1+\|v\|_{H_{\left(1-\alpha_{2}\right) / 3}}}\right)\right. \\
\cdot & {\left[1+\|\xi\|_{H_{\left(1-\alpha_{2}\right) / 3}}+\sup _{u \in[0, T]}\left\|O_{u}^{I}\right\|_{H_{\left(1-\alpha_{2}\right) / 3}}\right.} \\
& \left.\left.+\frac{T^{1-\alpha_{1}-\left(\left(1-\alpha_{2}\right) / 3\right)}}{1-\alpha_{1}-\left(\left(1-\alpha_{2}\right) / 3\right)}\left(\sup _{v \in H_{1 / 2}} \frac{\left\|P_{I} F(v)\right\|_{H_{-\alpha}}}{1+\|v\|_{H}^{2}}\right) \sup _{u \in[0, T]}\left\|X_{u}^{I}\right\|_{H}^{2}\right]^{2}\right]^{2} .
\end{aligned}
$$

Combining (210)-(213) and the assumption that $\sup _{I \in \mathcal{P}_{0}(\mathbb{H})} \sup _{u \in[0, T]}\left\|O_{u}^{I}\right\|_{H_{\iota}}<\infty$ therefore assures that for every $\kappa \in[1 / 2,1)$ with $\kappa \leq \iota$ it holds that

$$
\sup _{I \in \mathcal{P}_{0}(\mathbb{H})} \sup _{t \in[0, T]}\left\|X_{t}^{I}\right\|_{H_{\kappa}}<\infty .
$$

This, (215), and (217) establish (207). The proof of Lemma 5.3 is thus completed.

Lemma 5.4. Assume Setting 1.2 and let $T \in(0, \infty), \alpha \in(0,1), \gamma \in \mathbb{R}, \mathcal{Z} \in \mathcal{C}\left([0, T], H_{\gamma}\right)$. Then

(i) it holds for every $t \in[0, T]$ that $\int_{0}^{t}\left\|(t-u)^{\alpha-1} e^{(t-u) A} \mathcal{Z}_{u}\right\|_{H_{\gamma}} d u<\infty$ and

(ii) it holds that $\left([0, T] \ni t \mapsto \int_{0}^{t}(t-u)^{\alpha-1} e^{(t-u) A} \mathcal{Z}_{u} d u \in H_{\gamma}\right) \in \mathcal{C}\left([0, T], H_{\gamma}\right)$.

Proof of Lemma 5.4. Note that for every $t \in[0, T]$ it holds that

$$
\begin{aligned}
\int_{0}^{t}\left\|(t-u)^{\alpha-1} e^{(t-u) A} \mathcal{Z}_{u}\right\|_{H_{\gamma}} d u & \leq \int_{0}^{t}(t-u)^{\alpha-1}\left\|\mathcal{Z}_{u}\right\|_{H_{\gamma}} d u \\
& \leq \frac{t^{\alpha}}{\alpha} \sup _{u \in[0, T]}\left\|\mathcal{Z}_{u}\right\|_{H_{\gamma}}<\infty
\end{aligned}
$$


This establishes item (ii). Next observe that item (ii) ensures that there exists a function $Z:[0, T] \rightarrow$ $H_{\gamma}$ which satisfies for every $t \in[0, T]$ that

$$
Z_{t}=\int_{0}^{t}(t-u)^{\alpha-1} e^{(t-u) A} \mathcal{Z}_{u} d u
$$

Note that (221) and the triangle inequality show that for every $s \in[0, T], t \in[s, T]$ it holds that

$$
\begin{aligned}
& \left\|Z_{t}-Z_{s}\right\|_{H_{\gamma}} \\
& \leq\left\|\int_{s}^{t}(t-u)^{\alpha-1} e^{(t-u) A} \mathcal{Z}_{u} d u\right\|_{H_{\gamma}}+\left\|\int_{0}^{s}\left((t-u)^{\alpha-1} e^{(t-u) A}-(s-u)^{\alpha-1} e^{(s-u) A}\right) \mathcal{Z}_{u} d u\right\|_{H_{\gamma}} \\
& \leq \int_{s}^{t}(t-u)^{\alpha-1}\left\|e^{(t-u) A} \mathcal{Z}_{u}\right\|_{H_{\gamma}} d u+\int_{0}^{s}\left\|\left((t-u)^{\alpha-1} e^{(t-u) A}-(s-u)^{\alpha-1} e^{(s-u) A}\right) \mathcal{Z}_{u}\right\|_{H_{\gamma}} d u .
\end{aligned}
$$

Furthermore, observe that for every $s \in[0, T], t \in[s, T]$ it holds that

$$
\begin{aligned}
& \int_{s}^{t}(t-u)^{\alpha-1}\left\|e^{(t-u) A} \mathcal{Z}_{u}\right\|_{H_{\gamma}} d u \leq \int_{s}^{t}(t-u)^{\alpha-1}\left\|\mathcal{Z}_{u}\right\|_{H_{\gamma}} d u \\
& \leq\left[\sup _{u \in[0, T]}\left\|\mathcal{Z}_{u}\right\|_{H_{\gamma}}\right] \int_{s}^{t}(t-u)^{\alpha-1} d u \leq\left[\sup _{u \in[0, T]}\left\|\mathcal{Z}_{u}\right\|_{H_{\gamma}}\right] \frac{(t-s)^{\alpha}}{\alpha} .
\end{aligned}
$$

In addition, note that the triangle inequality assures that for every $s \in[0, T], t \in[s, T]$ it holds that

$$
\begin{aligned}
& \int_{0}^{s}\left\|\left((t-u)^{\alpha-1} e^{(t-u) A}-(s-u)^{\alpha-1} e^{(s-u) A}\right) \mathcal{Z}_{u}\right\|_{H_{\gamma}} d u \\
& \leq \int_{0}^{s}\left[(t-u)^{\alpha-1}\left\|\left(e^{(t-u) A}-e^{(s-u) A}\right) \mathcal{Z}_{u}\right\|_{H_{\gamma}}+\left((s-u)^{\alpha-1}-(t-u)^{\alpha-1}\right)\left\|e^{(s-u) A} \mathcal{Z}_{u}\right\|_{H_{\gamma}}\right] d u \\
& \leq \int_{0}^{s}(t-u)^{\alpha-1}\left\|e^{(s-u) A}\left(e^{(t-s) A}-\operatorname{Id}_{H_{\gamma}}\right) \mathcal{Z}_{u}\right\|_{H_{\gamma}} d u \\
& \quad+\int_{0}^{s}\left((s-u)^{\alpha-1}-(t-u)^{\alpha-1}\right)\left\|\mathcal{Z}_{u}\right\|_{H_{\gamma}} d u
\end{aligned}
$$

Next observe that the fact that for every $t \in(0, T], s \in(0, t), u \in[0, s)$ it holds that $(t-u)^{\alpha-1} \leq$ $(t-s)^{\alpha-1}$ proves that for every $s \in[0, T], t \in[s, T], \rho \in(1-\alpha, 1)$ it holds that

$$
\begin{aligned}
& \int_{0}^{s}(t-u)^{\alpha-1}\left\|e^{(s-u) A}\left(e^{(t-s) A}-\operatorname{Id}_{H_{\gamma}}\right) \mathcal{Z}_{u}\right\|_{H_{\gamma}} d u \\
& \leq \int_{0}^{s}(t-u)^{\alpha-1}\left\|(-A)^{\rho} e^{(s-u) A}\right\|_{L\left(H_{\gamma}\right)}\left\|(-A)^{-\rho}\left(e^{(t-s) A}-\operatorname{Id}_{H_{\gamma}}\right)\right\|_{L\left(H_{\gamma}\right)}\left\|\mathcal{Z}_{u}\right\|_{H_{\gamma}} d u \\
& \leq\left[\sup _{u \in[0, T]}\left\|\mathcal{Z}_{u}\right\|_{H_{\gamma}}\right] \int_{0}^{s}(t-u)^{\alpha-1}(s-u)^{-\rho}(t-s)^{\rho} d u \\
& \leq(t-s)^{\rho+\alpha-1}\left[\sup _{u \in[0, T]}\left\|\mathcal{Z}_{u}\right\|_{H_{\gamma}}\right] \int_{0}^{s}(s-u)^{-\rho} d u=(t-s)^{\rho+\alpha-1}\left[\sup _{u \in[0, T]}\left\|\mathcal{Z}_{u}\right\|_{H_{\gamma}}\right] \frac{s^{1-\rho}}{1-\rho} .
\end{aligned}
$$

Moreover, observe that the fact that for every $x, y \in[0, T], z \in[0,1]$ it holds that $\left|x^{z}-y^{z}\right| \leq|x-y|^{z}$ ensures that for every $t \in(0, T], s \in(0, t), u \in[0, s)$ it holds that

$$
(s-u)^{\alpha-1}-(t-u)^{\alpha-1} \leq \frac{(t-s)^{1-\alpha}}{(s-u)^{1-\alpha}(t-u)^{1-\alpha}} .
$$


This implies that for every $s \in[0, T], t \in[s, T], \varepsilon \in(0, \min \{\alpha /(2(1-\alpha)), 1 / 2,1-\alpha\})$ it holds that

$$
\begin{aligned}
& \int_{0}^{s}\left((s-u)^{\alpha-1}-(t-u)^{\alpha-1}\right) d u \leq(t-s)^{1-\alpha} \int_{0}^{s} \frac{d u}{(s-u)^{1-\alpha}(t-u)^{1-\alpha}} \\
& =(t-s)^{1-\alpha} \int_{0}^{s}(s-u)^{\alpha-1}(t-u)^{\alpha-1+\varepsilon}(t-u)^{-\varepsilon} d u \\
& \leq(t-s)^{1-\alpha} \int_{0}^{s}(s-u)^{\alpha-1}(t-s)^{\alpha-1+\varepsilon}(t-u)^{-\varepsilon} d u \\
& \leq(t-s)^{\varepsilon} \int_{0}^{s}(s-u)^{\alpha-1}(t-u)^{-\varepsilon} d u \\
& \leq(t-s)^{\varepsilon}\left[\int_{0}^{s}(s-u)^{(\alpha-1)(1+2 \varepsilon)} d u\right]^{1 /(1+2 \varepsilon)}\left[\int_{0}^{s}(t-u)^{-\varepsilon(1+2 \varepsilon) / 2 \varepsilon} d u\right]^{2 \varepsilon /(1+2 \varepsilon)} \\
& \leq(t-s)^{\varepsilon}\left[\frac{s^{1+(\alpha-1)(1+2 \varepsilon)}}{1+(\alpha-1)(1+2 \varepsilon)}\right]^{1 /(1+2 \varepsilon)}\left[\int_{0}^{t}(t-u)^{-\varepsilon-(1 / 2)} d u\right]^{2 \varepsilon /(1+2 \varepsilon)} \\
& =(t-s)^{\varepsilon}\left[\frac{s^{1+(\alpha-1)(1+2 \varepsilon)}}{1+(\alpha-1)(1+2 \varepsilon)}\right]^{1 /(1+2 \varepsilon)}\left[\frac{t^{(1 / 2)-\varepsilon}}{(1 / 2)-\varepsilon}\right]^{2 \varepsilon /(1+2 \varepsilon)} .
\end{aligned}
$$

Combining (222) -(225) therefore demonstrates that for every $s \in[0, T], t \in[s, T], \rho \in(1-\alpha, 1)$, $\varepsilon \in(0, \min \{\alpha /(2(1-\alpha)), 1 / 2,1-\alpha\})$ it holds that

$$
\begin{aligned}
& \left\|Z_{t}-Z_{s}\right\|_{H_{\gamma}} \leq \sup _{u \in[0, T]}\left\|\mathcal{Z}_{u}\right\|_{H_{\gamma}} \\
& \cdot\left[\frac{(t-s)^{\alpha}}{\alpha}+(t-s)^{\rho+\alpha-1} \frac{s^{1-\rho}}{1-\rho}+(t-s)^{\varepsilon}\left[\frac{\max \{T, 1\}}{1+(\alpha-1)(1+2 \varepsilon)}\right]^{1 /(1+2 \varepsilon)}\left[\frac{\max \{T, 1\}}{(1 / 2)-\varepsilon}\right]^{2 \varepsilon /(1+2 \varepsilon)}\right] .
\end{aligned}
$$

This establishes item (iii). The proof of Lemma 5.4 is thus completed.

Lemma 5.5. Assume Setting 1.2, let $T \in(0, \infty), \beta \in \mathbb{R}, \gamma \in(-\infty, 1 / 2+\beta), B \in \operatorname{HS}\left(H, H_{\beta}\right)$, let $(\Omega, \mathcal{F}, \mathbb{P})$ be a probability space, for every set $R$ and every function $f: \Omega \rightarrow R$ let $[f]_{\mathbb{P}, \mathcal{B}\left(H_{\gamma}\right)}=\{g \in$ $\mathcal{M}\left(\mathcal{F}, \mathcal{B}\left(H_{\gamma}\right)\right):(\exists D \in \mathcal{F}: \mathbb{P}(D)=0$ and $\left.\{\omega \in \Omega: f(\omega) \neq g(\omega)\} \subseteq D)\right\}$, and let $\left(W_{t}\right)_{t \in[0, T]}$ be an $\operatorname{Id}_{H}$-cylindrical Wiener process. Then there exists an up to indistinguishability unique stochastic process $O:[0, T] \times \Omega \rightarrow H_{\gamma}$ with continuous sample paths which satisfies for every $t \in[0, T]$ that $\left[O_{t}\right]_{\mathbb{P}, \mathcal{B}\left(H_{\gamma}\right)}=\int_{0}^{t} e^{(t-s) A} B d W_{s}$.

Proof of Lemma 5.5. Note that the fact that $\gamma-\beta<1 / 2$ ensures that for every $t \in[0, T]$ it holds that

$$
\begin{aligned}
& \int_{0}^{t}\left\|e^{(t-s) A} B\right\|_{\mathrm{HS}\left(H, H_{\gamma}\right)}^{2} d s=\int_{0}^{t}\left\|(-A)^{\min \{0, \gamma-\beta\}}(-A)^{\max \{0, \gamma-\beta\}} e^{(t-s) A} B\right\|_{\mathrm{HS}\left(H, H_{\beta}\right)}^{2} d s \\
& \leq\left\|(-A)^{\min \{0, \gamma-\beta\}}\right\|_{L(H)}^{2} \int_{0}^{t}\left\|(-A)^{\max \{0, \gamma-\beta\}} e^{(t-s) A}\right\|_{L(H)}^{2}\|B\|_{\mathrm{HS}\left(H, H_{\beta}\right)}^{2} d s \\
& \leq\left\|(-A)^{\min \{0, \gamma-\beta\}}\right\|_{L(H)}^{2}\|B\|_{\mathrm{HS}\left(H, H_{\beta}\right)}^{2} \int_{0}^{t}(t-s)^{-2 \max \{0, \gamma-\beta\}} d s \\
& =\left\|(-A)^{\min \{0, \gamma-\beta\}}\right\|_{L(H)}^{2}\|B\|_{\mathrm{HS}\left(H, H_{\beta}\right)}^{2} \frac{t^{1-2 \max \{0, \gamma-\beta\}}}{1-2 \max \{0, \gamma-\beta\}} .
\end{aligned}
$$

This shows that there exists a stochastic process $\mathbf{O}:[0, T] \times \Omega \rightarrow H_{\gamma}$ which satisfies for every $t \in[0, T]$ that

$$
\left[\mathbf{O}_{t}\right]_{\mathbb{P}, \mathcal{B}\left(H_{\gamma}\right)}=\int_{0}^{t} e^{(t-s) A} B d W_{s}
$$


Observe that (230) and the Burkholder-Davis-Gundy-type inequality in Da Prato \& Zabczyk [9, Lemma 7.7] prove that for every $p \in[2, \infty), s \in[0, T], t \in[s, T], \rho \in(0, \min \{1,1 / 2+\beta-\gamma\})$ it holds that

$$
\begin{aligned}
& \left\|\mathbf{O}_{t}-\mathbf{O}_{s}\right\|_{\mathcal{L}^{p}\left(\mathbb{P} ; H_{\gamma}\right)}=\left\|\int_{0}^{t} e^{(t-u) A} B d W_{u}-\int_{0}^{s} e^{(s-u) A} B d W_{u}\right\|_{L^{p}\left(\mathbb{P} ; H_{\gamma}\right)} \\
& \leq\left\|\int_{s}^{t} e^{(t-u) A} B d W_{u}\right\|_{L^{p}\left(\mathbb{P} ; H_{\gamma}\right)}+\left\|\int_{0}^{s} e^{(s-u) A}\left(e^{(t-s) A}-\operatorname{Id}_{H_{\beta}}\right) B d W_{u}\right\|_{L^{p}\left(\mathbb{P} ; H_{\gamma}\right)} \\
& \leq\left[\frac{p(p-1)}{2} \int_{s}^{t}\left\|(-A)^{\min \{0, \gamma-\beta\}}\right\|_{L(H)}^{2}\left\|(-A)^{\max \{0, \gamma-\beta\}} e^{(t-u) A}\right\|_{L(H)}^{2}\|B\|_{\mathrm{HS}\left(H, H_{\beta}\right)}^{2} d u\right]^{1 / 2} \\
& +\left[\frac{p(p-1)}{2} \int_{0}^{s}\left\|(-A)^{\min \{0, \rho+\gamma-\beta\}}\right\|_{L(H)}^{2}\left\|(-A)^{\max \{0, \rho+\gamma-\beta\}} e^{(s-u) A}\right\|_{L(H)}^{2}\right. \\
& \left.\cdot\left\|(-A)^{-\rho}\left(e^{(t-s) A}-\operatorname{Id}_{H}\right)\right\|_{L(H)}^{2}\|B\|_{\mathrm{HS}\left(H, H_{\beta}\right)}^{2} d u\right]^{1 / 2} \\
& \leq \frac{p}{\sqrt{2}}\|B\|_{\mathrm{HS}\left(H, H_{\beta}\right)}\left(\left\|(-A)^{\min \{0, \gamma-\beta\}}\right\|_{L(H)}\left[\int_{s}^{t}(t-u)^{-2 \max \{0, \gamma-\beta\}} d u\right]^{1 / 2}\right. \\
& \left.+\left\|(-A)^{\min \{0, \rho+\gamma-\beta\}}\right\|_{L(H)}\left[\int_{0}^{s}(s-u)^{-2 \max \{0, \rho+\gamma-\beta\}}(t-s)^{2 \rho} d u\right]^{1 / 2}\right) \\
& \leq \frac{p}{\sqrt{2}}\|B\|_{\mathrm{HS}\left(H, H_{\beta}\right)}\left(\left\|(-A)^{\min \{0, \gamma-\beta\}}\right\|_{L(H)}+\left\|(-A)^{\min \{0, \rho+\gamma-\beta\}}\right\|_{L(H)}\right) \\
& \cdot\left[\frac{(t-s)^{(1 / 2)-\max \{0, \gamma-\beta\}}}{\sqrt{1-2 \max \{0, \gamma-\beta\}}}+(t-s)^{\rho} \frac{s^{(1 / 2)-\max \{0, \rho+\gamma-\beta\}}}{\sqrt{1-2 \max \{0, \rho+\gamma-\beta\}}}\right] .
\end{aligned}
$$

The Kolmogorov-Chentsov theorem (cf., e.g., Kallenberg [24, Theorem 2.23]) therefore assures that there exists an up to indistinguishability unique stochastic process $O:[0, T] \times \Omega \rightarrow H_{\gamma}$ with continuous sample paths which satisfies for every $t \in[0, T]$ that $\left[O_{t}\right]_{\mathbb{P}, \mathcal{B}\left(H_{\gamma}\right)}=\int_{0}^{t} e^{(t-s) A} B d W_{s}$. The proof of Lemma 5.5 is thus completed.

Lemma 5.6. Assume Setting [1.2, let $T \in(0, \infty), I \subseteq \mathbb{H}, \beta \in \mathbb{R}, \gamma \in\left(-\infty, \frac{1}{2}+\beta\right)$, $\alpha \in$ $\left(0, \frac{1}{2}-\max \{0, \gamma-\beta\}\right), B \in \operatorname{HS}\left(H, H_{\beta}\right)$, let $\mathbb{B} \in L(H), P \in L\left(H_{\min \{0, \gamma\}}\right)$ satisfy for every $u, v \in H$ that $\langle B u, v\rangle_{H}=\langle u, \mathbb{B} v\rangle_{H}$ and $P v=\sum_{h \in I}\langle h, v\rangle_{H} h$, let $(\Omega, \mathcal{F}, \mathbb{P})$ be a probability space, for every set $R$ and every function $f: \Omega \rightarrow R$ let $[f]_{\mathbb{P}, \mathcal{B}\left(H_{\gamma}\right)}=\left\{g \in \mathcal{M}\left(\mathcal{F}, \mathcal{B}\left(H_{\gamma}\right)\right):(\exists D \in \mathcal{F}: \mathbb{P}(D)=\right.$ 0 and $\{\omega \in \Omega: f(\omega) \neq g(\omega)\} \subseteq D)\}$, let $\left(W_{t}\right)_{t \in[0, T]}$ be an $\operatorname{Id}_{H^{-}}$cylindrical Wiener process, and let $O:[0, T] \times \Omega \rightarrow H_{\gamma}$ be a stochastic process with continuous sample paths which satisfies for every $t \in[0, T]$ that $\left[O_{t}\right]_{\mathbb{P}, \mathcal{B}\left(H_{\gamma}\right)}=\int_{0}^{t} e^{(t-s) A} B d W_{s}$. Then it holds for every $p \in(1 / \alpha, \infty)$ that

$$
\left(\mathbb{E}\left[\sup _{t \in[0, T]}\left\|P O_{t}\right\|_{H_{\gamma}}^{p}\right]\right)^{1 / p} \leq T^{\alpha} 2^{\alpha-1}\left[\frac{p(p-1)}{p \alpha-1}\right]\left[\left(\int_{0}^{\infty} s^{-2 \alpha} e^{-s} d s\right) \sum_{h \in I}\|\mathbb{B} h\|_{H}^{2}\left|\mathfrak{v}_{h}\right|^{2(\alpha+\gamma)-1}\right]^{1 / 2} .
$$

Proof of Lemma 5.6. Note that for every $t \in[0, T]$ it holds that

$$
\begin{aligned}
& \int_{0}^{t}(t-u)^{-2 \alpha}\left\|e^{(t-u) A} B\right\|_{\mathrm{HS}\left(U, H_{\gamma}\right)}^{2} d u \\
& \leq \int_{0}^{t}(t-u)^{-2 \alpha}\left\|(-A)^{\max \{0, \gamma-\beta\}} e^{(t-u) A}\right\|_{L(H)}^{2}\left\|(-A)^{\min \{0, \gamma-\beta\}}\right\|_{L(H)}^{2}\|B\|_{\mathrm{HS}\left(U, H_{\beta}\right)}^{2} d u \\
& \leq\left\|(-A)^{\min \{0, \gamma-\beta\}}\right\|_{L(H)}^{2}\|B\|_{\mathrm{HS}\left(U, H_{\beta}\right)}^{2} \int_{0}^{t}(t-u)^{-2[\alpha+\max \{0, \gamma-\beta\}]} d u<\infty .
\end{aligned}
$$


This ensures that there exists a stochastic process $Z:[0, T] \times \Omega \rightarrow H_{\gamma}$ which satisfies for every $t \in[0, T]$ that

$$
\left[Z_{t}\right]_{\mathbb{P}, \mathcal{B}\left(H_{\gamma}\right)}=\int_{0}^{t}(t-u)^{-\alpha} e^{(t-u) A} B d W_{u} .
$$

Note that (234) and the triangle inequality prove that for every $p \in[2, \infty), t \in(0, T], s \in[0, t)$ it holds that

$$
\begin{aligned}
\left\|Z_{t}-Z_{s}\right\|_{\mathcal{L}^{p}\left(\mathbb{P} ; H_{\gamma}\right)}= & \left\|\int_{0}^{s}\left((t-u)^{-\alpha} e^{(t-u) A}-(s-u)^{-\alpha} e^{(s-u) A}\right) B d W_{u}\right\|_{L^{p}\left(\mathbb{P} ; H_{\gamma}\right)} \\
& +\left\|\int_{s}^{t}(t-u)^{-\alpha} e^{(t-u) A} B d W_{u}\right\|_{L^{p}\left(\mathbb{P} ; H_{\gamma}\right)} \\
\leq & \left\|\int_{0}^{s}(t-u)^{-\alpha}\left(e^{(t-u) A}-e^{(s-u) A}\right) B d W_{u}\right\|_{L^{p}\left(\mathbb{P} ; H_{\gamma}\right)} \\
& +\left\|\int_{0}^{s} e^{(s-u) A}\left((t-u)^{-\alpha}-(s-u)^{-\alpha}\right) B d W_{u}\right\|_{L^{p}\left(\mathbb{P} ; H_{\gamma}\right)} \\
& +\left\|\int_{s}^{t}(t-u)^{-\alpha} e^{(t-u) A} B d W_{u}\right\|_{L^{p}\left(\mathbb{P} ; H_{\gamma}\right)}
\end{aligned}
$$

The Burkholder-Davis-Gundy-type inequality in Da Prato \& Zabczyk [9, Lemma 7.7] hence shows that for every $p \in[2, \infty), t \in(0, T], s \in[0, t)$ it holds that

$$
\begin{aligned}
& \left\|Z_{t}-Z_{s}\right\|_{\mathcal{L}^{p}\left(\mathbb{P} ; H_{\gamma}\right)} \leq \frac{\sqrt{p(p-1)}}{\sqrt{2}}\left[\int_{0}^{s}(t-u)^{-2 \alpha}\left\|(-A)^{\gamma-\beta}\left(e^{(t-u) A}-e^{(s-u) A}\right) B\right\|_{\mathrm{HS}\left(H, H_{\beta}\right)}^{2} d u\right]^{1 / 2} \\
& +\frac{\sqrt{p(p-1)}}{\sqrt{2}}\left[\int_{0}^{s}\left((s-u)^{-\alpha}-(t-u)^{-\alpha}\right)^{2}\left\|(-A)^{\min \{0, \gamma-\beta\}}\right\|_{L(H)}^{2}\right. \\
& \left.\cdot\left\|(-A)^{\max \{0, \gamma-\beta\}} e^{(s-u) A}\right\|_{L(H)}^{2}\|B\|_{\mathrm{HS}\left(H, H_{\beta}\right)}^{2} d u\right]^{1 / 2} \\
& +\frac{\sqrt{p(p-1)}}{\sqrt{2}}\left[\int_{s}^{t}(t-u)^{-2 \alpha}\left\|(-A)^{\min \{0, \gamma-\beta\}}\right\|_{L(H)}^{2}\left\|(-A)^{\max \{0, \gamma-\beta\}} e^{(t-u) A}\right\|_{L(H)}^{2}\|B\|_{\mathrm{HS}\left(H, H_{\beta}\right)}^{2} d u\right]^{1 / 2} \cdot
\end{aligned}
$$

Therefore, we obtain that for every $p \in[2, \infty), \varepsilon \in\left(0, \frac{1}{2}+\beta-\alpha-\gamma\right), t \in(0, T], s \in[0, t)$ it holds 
that

$$
\begin{aligned}
& \left\|Z_{t}-Z_{s}\right\|_{\mathcal{L}^{p}\left(\mathbb{P} ; H_{\gamma}\right)} \leq \frac{\sqrt{p(p-1)}}{\sqrt{2}}\|B\|_{\mathrm{HS}\left(H, H_{\beta}\right)} \\
& \cdot\left(\left[\int_{0}^{s}(t-u)^{-2 \alpha}\left\|(-A)^{\min \{0, \varepsilon+\alpha+\gamma-\beta\}}\right\|_{L(H)}\left\|(-A)^{\max \{0, \varepsilon+\alpha+\gamma-\beta\}} e^{(s-u) A}\right\|_{L(H)}^{2}\right.\right. \\
& \left.\quad \cdot\left\|(-A)^{-\varepsilon-\alpha}\left(e^{(t-s) A}-\operatorname{Id}_{H}\right)\right\|_{L(H)}^{2} d u\right]^{1 / 2} \\
& +\left\|(-A)^{\min \{0, \gamma-\beta\}}\right\|_{L(H)}\left[\int_{0}^{s}\left((s-u)^{-\alpha}-(t-u)^{-\alpha}\right)^{2}(s-u)^{-2 \max \{0, \gamma-\beta\}} d u\right]^{1 / 2} \\
& \left.+\left\|(-A)^{\min \{0, \gamma-\beta\}}\right\|_{L(H)}\left[\int_{s}^{t}(t-u)^{-2 \alpha-2 \max \{0, \gamma-\beta\}} d u\right]^{1 / 2}\right) \\
& \leq \frac{\sqrt{p(p-1)}}{\sqrt{2}}\|B\|_{\operatorname{HS}\left(H, H_{\beta}\right)} \\
& \cdot\left(\left\|(-A)^{\min \{0, \varepsilon+\alpha+\gamma-\beta\}}\right\|_{L(H)}\left[\int_{0}^{s}(t-u)^{-2 \alpha}(s-u)^{-2 \max \{0, \varepsilon+\alpha+\gamma-\beta\}}(t-s)^{2 \varepsilon+2 \alpha} d u\right]^{1 / 2}\right. \\
& +\left\|(-A)^{\min \{0, \gamma-\beta\}}\right\|_{L(H)}\left[\int_{0}^{s}\left((s-u)^{-\alpha}-(t-u)^{-\alpha}\right)^{2}(s-u)^{-2 \max \{0, \gamma-\beta\}} d u\right]^{1 / 2} \\
& \left.+\left\|(-A)^{\min \{0, \gamma-\beta\}}\right\|_{L(H)}\left[\frac{(t-s)^{1-2 \alpha-2 \max \{0, \gamma-\beta\}}}{1-2 \alpha-2 \max \{0, \gamma-\beta\}}\right]^{1 / 2}\right) .
\end{aligned}
$$

In addition, note that for every $\varepsilon \in\left(0, \frac{1}{2}+\beta-\alpha-\gamma\right), t \in(0, T], s \in[0, t)$ it holds that

$$
\begin{aligned}
& \int_{0}^{s}(t-u)^{-2 \alpha}(s-u)^{-2 \max \{0, \varepsilon+\alpha+\gamma-\beta\}}(t-s)^{2 \varepsilon+2 \alpha} d u \\
& \leq \int_{0}^{s}(t-s)^{-2 \alpha}(s-u)^{-2 \max \{0, \varepsilon+\alpha+\gamma-\beta\}}(t-s)^{2 \varepsilon+2 \alpha} d u \\
& \leq(t-s)^{2 \varepsilon} \frac{s^{1-2 \max \{0, \varepsilon+\alpha+\gamma-\beta\}}}{1-2 \max \{0, \varepsilon+\alpha+\gamma-\beta\}} \leq(t-s)^{2 \varepsilon} \frac{\max \{T, 1\}}{1-2 \max \{0, \varepsilon+\alpha+\gamma-\beta\}} .
\end{aligned}
$$

Next observe that the fact that for every $x, y \in[0, T], z \in[0,1]$ it holds that $\left|x^{z}-y^{z}\right| \leq|x-y|^{z}$ ensures that for every $t \in(0, T], s \in(0, t), u \in(0, s)$ it holds that

$$
(s-u)^{-\alpha}-(t-u)^{-\alpha} \leq \frac{(t-s)^{\alpha}}{(s-u)^{\alpha}(t-u)^{\alpha}} .
$$

Hölder's inequality hence proves that for every $\varepsilon \in\left(0, \min \left\{\frac{1}{8(\alpha+\max \{0, \gamma-\beta\})}-\frac{1}{4}, \frac{1}{4}, \alpha\right\}\right), t \in(0, T]$, $s \in[0, t)$ it holds that

$$
\begin{aligned}
& \int_{0}^{s}\left((s-u)^{-\alpha}-(t-u)^{-\alpha}\right)^{2}(s-u)^{-2 \max \{0, \gamma-\beta\}} d u \leq \int_{0}^{s} \frac{(t-s)^{2 \alpha}}{(s-u)^{2 \alpha}(t-u)^{2 \alpha}}(s-u)^{-2 \max \{0, \gamma-\beta\}} d u \\
& =(t-s)^{2 \varepsilon} \int_{0}^{s}(s-u)^{-2 \alpha-2 \max \{0, \gamma-\beta\}}(t-u)^{-2 \alpha}(t-s)^{2 \alpha-2 \varepsilon} d u \\
& \leq(t-s)^{2 \varepsilon} \int_{0}^{s}(s-u)^{-2 \alpha-2 \max \{0, \gamma-\beta\}}(t-u)^{-2 \varepsilon} d u \\
& \leq(t-s)^{2 \varepsilon}\left(\int_{0}^{s}(s-u)^{-2(\alpha+\max \{0, \gamma-\beta\})(1+4 \varepsilon)} d u\right)^{1 /(1+4 \varepsilon)}\left(\int_{0}^{s}(t-u)^{-2 \varepsilon(1+4 \varepsilon) / 4 \varepsilon} d u\right)^{4 \varepsilon /(1+4 \varepsilon)} \\
& =(t-s)^{2 \varepsilon}\left(\frac{s^{1-2(\alpha+\max \{0, \gamma-\beta\})(1+4 \varepsilon)}}{1-2(\alpha+\max \{0, \gamma-\beta\})(1+4 \varepsilon)}\right)^{1 /(1+4 \varepsilon)}\left(\frac{t^{(1 / 2)-2 \varepsilon}-(t-s)^{(1 / 2)-2 \varepsilon}}{(1 / 2)-2 \varepsilon}\right)^{4 \varepsilon /(1+4 \varepsilon)} \\
& \leq(t-s)^{2 \varepsilon} \frac{\max \{T, 1\}}{(1-2(\alpha+\max \{0, \gamma-\beta\})(1+4 \varepsilon))^{1 /(1+4 \varepsilon)((1 / 2)-2 \varepsilon)^{4 \varepsilon /(1+4 \varepsilon)}}}
\end{aligned}
$$


Combining this, (237), and (238) demonstrates that for every $\varepsilon \in\left(0, \min \left\{\frac{1}{8(\alpha+\max \{0, \gamma-\beta\})}-\right.\right.$ $\left.\left.\frac{1}{4}, \frac{1}{4}, \alpha, \frac{1}{2}+\beta-\alpha-\gamma\right\}\right), p \in[2, \infty)$ it holds that

$$
\sup _{t \in(0, T], s \in[0, t)} \frac{\left\|Z_{t}-Z_{s}\right\|_{\mathcal{L}^{p}\left(\mathbb{P} ; H_{\gamma}\right)}}{|t-s|^{\varepsilon}}<\infty .
$$

The Kolmogorov-Chentsov theorem (cf., e.g., Kallenberg [24, Theorem 2.23]) therefore assures that there exists a stochastic process $\mathcal{Z}:[0, T] \times \Omega \rightarrow H_{\gamma}$ with continuous sample paths which satisfies for every $t \in[0, T]$ that

$$
\mathbb{P}\left(\mathcal{Z}_{t}=Z_{t}\right)=1 \text {. }
$$

Next note that the fact that $0<\alpha<\frac{1}{2}-\max \{0, \gamma-\beta\}$ ensures that for every $t \in[0, T]$ it holds that

$$
\begin{aligned}
& \int_{0}^{t}(t-s)^{\alpha-1}\left[\int_{0}^{s}(s-u)^{-2 \alpha} \mathbb{E}\left[\left\|e^{(t-u) A} P B\right\|_{\mathrm{HS}\left(H, H_{\gamma}\right)}^{2} d u\right]^{1 / 2} d s\right. \\
& =\int_{0}^{t}(t-s)^{\alpha-1}\left[\int_{0}^{s}(s-u)^{-2 \alpha}\left\|(-A)^{\min \{0, \gamma-\beta\}+\max \{0, \gamma-\beta\}} e^{(t-u) A} P B\right\|_{\mathrm{HS}\left(H, H_{\beta}\right)}^{2} d u\right]^{1 / 2} d s \\
& \leq\|B\|_{\mathrm{HS}\left(H, H_{\beta}\right)}\left\|(-A)^{\min \{0, \gamma-\beta\}}\right\|_{L(H)} \int_{0}^{t}(t-s)^{\alpha-1}\left[\int_{0}^{s}(s-u)^{-2(\alpha+\max \{0, \gamma-\beta\})} d u\right]^{1 / 2} d s \\
& =\|B\|_{\mathrm{HS}\left(H, H_{\beta}\right)}\left\|(-A)^{\min \{0, \gamma-\beta\}}\right\|_{L(H)} \frac{1}{\sqrt{1-2(\alpha+\max \{0, \gamma-\beta\})}} \int_{0}^{t}(t-s)^{\alpha-1} s^{(1 / 2)-(\alpha+\max \{0, \gamma-\beta\})} d s \\
& \leq\|B\|_{\mathrm{HS}\left(H, H_{\beta}\right)}\left\|(-A)^{\min \{0, \gamma-\beta\}}\right\|_{L(H)} \frac{[\max \{T, 1\}]^{1 / 2}}{\sqrt{1-2(\alpha+\max \{0, \gamma-\beta\})}} \frac{t^{\alpha}}{\alpha}<\infty .
\end{aligned}
$$

Combining (234), (242), the fact that $\mathcal{Z}:[0, T] \times \Omega \rightarrow H_{\gamma}$ has continuous sample paths, item (ii) of Lemma 5.4 (with $T=T, \alpha=\alpha, \gamma=\gamma, \mathcal{Z}=\left([0, T] \ni t \mapsto P \mathcal{Z}_{t}(\omega) \in H_{\gamma}\right.$ ) for $\omega \in \Omega$ in the notation of item (ii) of Lemma 5.4), and, e.g., Da Prato \& Zabczyk [11, Theorem 5.10] therefore establishes that for every $t \in[0, T]$ it holds that

$$
\int_{0}^{t} e^{(t-s) A} P B d W_{s}=\left[\frac{\sin (\alpha \pi)}{\pi} \int_{0}^{t}(t-s)^{\alpha-1} e^{(t-s) A} P \mathcal{Z}_{s} d s\right]_{\mathbb{P}, \mathcal{B}\left(H_{\gamma}\right)} .
$$

This, the fact that $\mathcal{Z}:[0, T] \times \Omega \rightarrow H_{\gamma}$ has continuous sample paths, and Lemma 5.4 (with $T=T$, $\alpha=\alpha, \gamma=\gamma, \mathcal{Z}=\left([0, T] \ni t \mapsto P \mathcal{Z}_{t}(\omega) \in H_{\gamma}\right)$ for $\omega \in \Omega$ in the notation of Lemma 5.4) imply that for every $\omega \in \Omega, p \in[1, \infty)$ it holds that $\left([0, T] \ni t \mapsto \int_{0}^{t}(t-s)^{\alpha-1} e^{(t-s) A} P \mathcal{Z}_{s}(\omega) d s \in H_{\gamma}\right) \in$ $\mathcal{C}\left([0, T], H_{\gamma}\right)$ and

$$
\begin{aligned}
\mathbb{E}\left[\sup _{t \in[0, T]}\left\|P O_{t}\right\|_{H_{\gamma}}^{p}\right] & =\mathbb{E}\left[\sup _{t \in[0, T]}\left\|\frac{\sin (\alpha \pi)}{\pi} \int_{0}^{t}(t-s)^{\alpha-1} e^{(t-s) A} P \mathcal{Z}_{s} d s\right\|_{H_{\gamma}}^{p}\right] \\
& \leq \mathbb{E}\left[\sup _{t \in[0, T]}\left(\int_{0}^{t}(t-s)^{\alpha-1}\left\|e^{(t-s) A} P \mathcal{Z}_{s}\right\|_{H_{\gamma}} d s\right)^{p}\right] .
\end{aligned}
$$

Hölder's inequality and Tonelli's theorem hence prove that for every $p \in(1 / \alpha, \infty)$ it holds that

$$
\begin{aligned}
& \mathbb{E}\left[\sup _{t \in[0, T]}\left\|P O_{t}\right\|_{H_{\gamma}}^{p}\right] \leq \mathbb{E}\left[\sup _{t \in[0, T]}\left(\int_{0}^{t}(t-s)^{\alpha-1}\left\|P \mathcal{Z}_{s}\right\|_{H_{\gamma}} d s\right)^{p}\right] \\
& \leq \mathbb{E}\left[\sup _{t \in[0, T]}\left\{\left(\int_{0}^{t}(t-s)^{\frac{p(\alpha-1)}{p-1}} d s\right)^{p-1}\left(\int_{0}^{t}\left\|P \mathcal{Z}_{s}\right\|_{H_{\gamma}}^{p} d s\right)\right\}\right] \\
& \leq\left(\frac{t^{1+(p(\alpha-1) /(p-1))}}{1+(p(\alpha-1) /(p-1))}\right)^{p-1} \int_{0}^{T} \mathbb{E}\left[\left\|P \mathcal{Z}_{s}\right\|_{H_{\gamma}}^{p}\right] d s \leq\left(\frac{p-1}{p \alpha-1}\right)^{p-1} T^{p \alpha} \sup _{s \in[0, T]} \mathbb{E}\left[\left\|P \mathcal{Z}_{s}\right\|_{H_{\gamma}}^{p}\right] .
\end{aligned}
$$


In addition, observe that the Burkholder-Davis-Gundy-type inequality in Da Prato \& Zabczyk [9, Lemma 7.7] shows that for every $p \in(1 / \alpha, \infty), t \in[0, T]$ it holds that

$$
\begin{aligned}
\left\|P \mathcal{Z}_{t}\right\|_{\mathcal{L}^{p}\left(\mathbb{P} ; H_{\gamma}\right)}^{2} & =\left\|\int_{0}^{t}(t-u)^{-\alpha} e^{(t-u) A} P B d W_{u}\right\|_{L^{p}\left(\mathbb{P} ; H_{\gamma}\right)}^{2} \\
& \leq \frac{p(p-1)}{2} \int_{0}^{t}(t-u)^{-2 \alpha}\left\|e^{(t-u) A} P B\right\|_{\mathrm{HS}\left(H, H_{\gamma}\right)}^{2} d u .
\end{aligned}
$$

Tonelli's theorem therefore implies that for every $p \in(1 / \alpha, \infty), t \in[0, T]$ it holds that

$$
\begin{aligned}
& \left\|P \mathcal{Z}_{t}\right\|_{\mathcal{L}^{p}\left(\mathbb{P} ; H_{\gamma}\right)}^{2} \leq \frac{p(p-1)}{2} \int_{0}^{t}(t-u)^{-2 \alpha}\left\|(-A)^{\gamma} e^{(t-u) A} P B\right\|_{\mathrm{HS}(H)}^{2} d u \\
& =\frac{p(p-1)}{2} \int_{0}^{t}(t-u)^{-2 \alpha}\left\|\mathbb{B}(-A)^{\gamma} P e^{(t-u) A}\right\|_{\mathrm{HS}(H)}^{2} d u \\
& =\frac{p(p-1)}{2} \int_{0}^{t}(t-u)^{-2 \alpha} \sum_{h \in I}\left\|\mathbb{B}(-A)^{\gamma} e^{(t-u) A} h\right\|_{H}^{2} d u \\
& =\frac{p(p-1)}{2} \int_{0}^{t}(t-u)^{-2 \alpha} \sum_{h \in I}\|\mathbb{B} h\|_{H}^{2}\left|\mathfrak{v}_{h}\right|^{2 \gamma} e^{2(t-u) \mathfrak{v}_{h}} d u \\
& =\frac{p(p-1)}{2} \sum_{h \in I}\|\mathbb{B} h\|_{H}^{2} \int_{0}^{t}(t-u)^{-2 \alpha} e^{2(t-u) \mathfrak{v}_{h}}\left|\mathfrak{v}_{h}\right|^{2 \gamma} d u \\
& =\frac{p(p-1)}{2} \sum_{h \in I}\|\mathbb{B} h\|_{H}^{2}\left(\int_{0}^{2\left|\mathfrak{v}_{h}\right| t} s^{-2 \alpha} e^{-s} d s\right) 2^{2 \alpha-1}\left(\left|\mathfrak{v}_{h}\right|\right)^{2(\alpha+\gamma)-1} \\
& \leq 2^{2 \alpha-2} p^{2}\left(\int_{0}^{\infty} s^{-2 \alpha} e^{-s} d s\right) \sum_{h \in I}\|\mathbb{B} h\|_{H}^{2}\left|\mathfrak{v}_{h}\right|^{2(\alpha+\gamma)-1} .
\end{aligned}
$$

Combining this with (246) ensures that

$$
\begin{aligned}
& \left(\mathbb{E}\left[\sup _{t \in[0, T]}\left\|P O_{t}\right\|_{H_{\gamma}}^{p}\right]\right)^{1 / p} \\
& \leq 2^{\alpha-1} p\left(\frac{p-1}{p \alpha-1}\right)^{(p-1) / p} T^{\alpha}\left[\left(\int_{0}^{\infty} s^{-2 \alpha} e^{-s} d s\right) \sum_{h \in I}\|\mathbb{B} h\|_{H}^{2}\left|\mathfrak{v}_{h}\right|^{2(\alpha+\gamma)-1}\right]^{1 / 2} .
\end{aligned}
$$

The proof of Lemma 5.6 is thus completed.

Lemma 5.7. Let $\left(V,\|\cdot\|_{V}\right)$ be an $\mathbb{R}$-Banach space, let $(\Omega, \mathcal{F}, \mathbb{P})$ be a probability space, let $\alpha \in$ $(0, \infty)$, and let $Z_{n}: \Omega \rightarrow V, n \in \mathbb{N}$, be $\mathcal{F} / \mathcal{B}(V)$-measurable functions which satisfy for every $p \in[1, \infty)$ that $\sup _{n \in \mathbb{N}}\left(n^{\alpha}\left\|Z_{n}\right\|_{\mathcal{L}^{p}(\mathbb{P} ; V)}\right)<\infty$. Then it holds for every $\varepsilon \in(0, \infty), p \in[1, \infty)$ that

$$
\mathbb{P}\left(\sup _{n \in \mathbb{N}}\left(n^{\alpha-\varepsilon}\left\|Z_{n}\right\|_{V}\right)<\infty\right)=1 \quad \text { and } \quad \mathbb{E}\left[\left(\sup _{n \in \mathbb{N}}\left(n^{\alpha-\varepsilon}\left\|Z_{n}\right\|_{V}\right)\right)^{p}\right]<\infty .
$$

Proof of Lemma 5.7. Observe that for every $\varepsilon, \delta \in(0, \infty), p \in(\max \{1 / \varepsilon, 1\}, \infty)$ it holds that

$$
\begin{aligned}
& \mathbb{E}\left[\left(\sup _{n \in \mathbb{N}}\left(n^{\alpha-\varepsilon}\left\|Z_{n}\right\|_{V}\right)\right)^{p}\right]=\mathbb{E}\left[\sup _{n \in \mathbb{N}}\left(n^{p(\alpha-\varepsilon)}\left\|Z_{n}\right\|_{V}^{p}\right)\right] \\
& \leq \sum_{n=1}^{\infty} n^{p(\alpha-\varepsilon)} \mathbb{E}\left[\left\|Z_{n}\right\|_{V}^{p}\right] \leq\left(\sup _{n \in \mathbb{N}}\left(n^{\alpha}\left\|Z_{n}\right\|_{\mathcal{L}^{p}(\mathbb{P} ; V)}\right)\right)^{p} \sum_{n=1}^{\infty} n^{-p \varepsilon}<\infty .
\end{aligned}
$$

Jensen's inequality therefore demonstrates that for every $\varepsilon \in(0, \infty), p \in[1, \infty)$ it holds that

$$
\mathbb{E}\left[\left(\sup _{n \in \mathbb{N}}\left(n^{\alpha-\varepsilon}\left\|Z_{n}\right\|_{V}\right)\right)^{p}\right]<\infty
$$

This establishes (250). The proof of Lemma 5.7 is thus completed. 
Lemma 5.8. Assume Setting 4.1, let $T \in(0, \infty), \beta \in \mathbb{R}, \gamma \in(-\infty, 1 / 2+\beta), B \in \operatorname{HS}\left(H, H_{\beta}\right)$, let $\mathcal{P}(\mathbb{H})$ be the power set of $\mathbb{H}$, let $\left(P_{I}\right)_{I \in \mathcal{P}(\mathbb{H})} \subseteq L\left(H_{\min \{0, \gamma\}}\right)$ satisfy for every $I \in \mathcal{P}(\mathbb{H}), v \in H_{\min \{0, \gamma\}}$ that $P_{I}(v)=\sum_{h \in I}\left\langle(-A)^{-\min \{0, \gamma\}} h,(-A)^{\min \{0, \gamma\}} v\right\rangle_{H} h$, let $(\Omega, \mathcal{F}, \mathbb{P})$ be a probability space, let $\left(W_{t}\right)_{t \in[0, T]}$ be an $\operatorname{Id}_{H}$-cylindrical Wiener process, and let $O:[0, T] \times \Omega \rightarrow H_{\gamma}$ be a stochastic process with continuous sample paths which satisfies for every $t \in[0, T]$ that $\left[O_{t}\right]_{\mathbb{P}, \mathcal{B}\left(H_{\gamma}\right)}=\int_{0}^{t} e^{(t-s) A} B d W_{s}$. Then

$$
\mathbb{P}\left(\forall \eta \in(-\infty, 1+2(\beta-\gamma)): \sup _{n \in \mathbb{N}}\left(n^{\eta} \sup _{t \in[0, T]}\left\|P_{\mathbb{H} \backslash\left\{e_{1}, \ldots, e_{n}\right\}} O_{t}\right\|_{H_{\gamma}}\right)<\infty\right)=1 .
$$

Proof of Lemma 5.8. Throughout this proof let $\mathbb{B} \in L(H)$ satisfy for every $u, v \in H$ that $\langle B u, v\rangle_{H}=\langle u, \mathbb{B} v\rangle_{H}$ and let $\left(I_{n}\right)_{n \in \mathbb{N}} \subseteq \mathbb{H}$ satisfy for every $n \in \mathbb{N}$ that $I_{n}=\left\{e_{1}, \ldots, e_{n}\right\}$. Note that Lemma 5.6 (with $T=T, I=\mathbb{H} \backslash I_{n}, \beta=\beta, \gamma=\gamma, \alpha=\alpha, B=B, \mathbb{B}=\mathbb{B}, P=P_{\mathbb{H} \backslash I_{n}}$, $(\Omega, \mathcal{F}, \mathbb{P})=(\Omega, \mathcal{F}, \mathbb{P}),\left(W_{t}\right)_{t \in[0, T]}=\left(W_{t}\right)_{t \in[0, T]}, O=O$ for $n \in \mathbb{N}, \alpha \in\left(0, \frac{1}{2}-\max \{0, \gamma-\beta\}\right)$ in the notation of Lemma (5.6) ensures that for every $n \in \mathbb{N}, \alpha \in\left(0, \frac{1}{2}-\max \{0, \gamma-\beta\}\right), p \in(1 / \alpha, \infty)$ it holds that

$$
\begin{aligned}
& \left(\mathbb{E}\left[\sup _{t \in[0, T]}\left\|P_{\mathbb{H} \backslash I_{n}} O_{t}\right\|_{H_{\gamma}}^{p}\right]\right)^{1 / p} \\
& \leq 2^{\alpha-1} \frac{p(p-1)}{p \alpha-1} T^{\alpha}\left[\left(\int_{0}^{\infty} s^{-2 \alpha} e^{-s} d s\right) \sum_{h \in \mathbb{H} \backslash I_{n}}\|\mathbb{B} h\|_{H}^{2}\left|\mathfrak{v}_{h}\right|^{2(\alpha+\gamma)-1}\right]^{1 / 2} \\
& \leq 2^{\alpha-1} \frac{p(p-1)}{p \alpha-1} T^{\alpha}\left[\left(\int_{0}^{\infty} s^{-2 \alpha} e^{-s} d s\right) \sum_{h \in \mathbb{H}}\|\mathbb{B} h\|_{H}^{2}\left|\mathfrak{v}_{h}\right|^{2 \beta}\right]^{1 / 2}\left(\sup _{h \in \mathbb{H} \backslash I_{n}}\left(\left|\mathfrak{v}_{h}\right|^{\alpha+\gamma-\beta-(1 / 2)}\right)\right) \\
& =2^{\alpha-1} \frac{p(p-1)}{p \alpha-1} T^{\alpha}\left[\left(\int_{0}^{\infty} s^{-2 \alpha} e^{-s} d s\right) \sum_{h \in \mathbb{H}}\left\|\mathbb{B}(-A)^{\beta} h\right\|_{H}^{2}\right]^{1 / 2}\left[\sqrt{\left|c_{0}\right|} \pi(n+1)\right]^{2(\alpha+\gamma-\beta)-1} \\
& =2^{\alpha-1} \frac{p(p-1)}{p \alpha-1} T^{\alpha}\left[\left(\int_{0}^{\infty} s^{-2 \alpha} e^{-s} d s\right)\left\|\mathbb{B}(-A)^{\beta}\right\|_{\mathrm{HS}(H)}^{2}\right]^{1 / 2}\left[\sqrt{\left|c_{0}\right|} \pi(n+1)\right]^{2(\alpha+\gamma-\beta)-1} \\
& =2^{\alpha-1} \frac{p(p-1)}{p \alpha-1} T^{\alpha}\left[\left(\int_{0}^{\infty} s^{-2 \alpha} e^{-s} d s\right)\|B\|_{\mathrm{HS}\left(H, H_{\beta}\right)}^{2}\right]^{1 / 2}\left[\sqrt{\left|c_{0}\right|} \pi(n+1)\right]^{2(\alpha+\gamma-\beta)-1} .
\end{aligned}
$$

Jensen's inequality hence implies that for every $\alpha \in\left(0, \frac{1}{2}-\max \{0, \gamma-\beta\}\right), p \in[1, \infty)$ it holds that

$$
\sup _{n \in \mathbb{N}}\left\{n^{1+2(\beta-\alpha-\gamma)}\left(\mathbb{E}\left[\sup _{t \in[0, T]}\left\|P_{\mathbb{H} \backslash I_{n}} O_{t}\right\|_{H_{\gamma}}^{p}\right]\right)^{1 / p}\right\}<\infty .
$$

Lemma $5.7\left(\right.$ with $V=\mathbb{R},(\Omega, \mathcal{F}, \mathbb{P})=(\Omega, \mathcal{F}, \mathbb{P}), \alpha=1+2(\beta-\alpha-\gamma), Z_{n}=\sup _{t \in[0, T]}\left\|P_{\mathbb{H} \backslash I_{n}} O_{t}\right\|_{H_{\gamma}}$ for $n \in \mathbb{N}, \alpha \in\left(0, \frac{1}{2}-\max \{0, \gamma-\beta\}\right)$ in the notation of Lemma 5.7) therefore shows that for every $\alpha \in\left(0, \frac{1}{2}-\max \{0, \gamma-\beta\}\right), \eta \in(0,1+2(\beta-\alpha-\gamma))$ it holds that

$$
\mathbb{P}\left(\sup _{n \in \mathbb{N}}\left(n^{\eta} \sup _{t \in[0, T]}\left\|P_{\mathbb{H} \backslash I_{n}} O_{t}\right\|_{H_{\gamma}}\right)<\infty\right)=1 .
$$

This completes the proof of Lemma 5.8,

Lemma 5.9. Assume Setting 4.1, let $T \in(0, \infty), \beta \in \mathbb{R}, \gamma \in(-\infty, 1 / 2+\beta), B \in \operatorname{HS}\left(H, H_{\beta}\right)$, let $(\Omega, \mathcal{F}, \mathbb{P})$ be a probability space with a normal filtration $\left(\mathbb{F}_{t}\right)_{t \in[0, T]}$, let $\left(W_{t}\right)_{t \in[0, T]}$ be an $\operatorname{Id}_{H^{-}}$ cylindrical $\left(\mathbb{F}_{t}\right)_{t \in[0, T]}$-Wiener process, let $\xi \in \mathcal{M}\left(\mathbb{F}_{0}, \mathcal{B}(H)\right)$, let $\mathcal{P}(\mathbb{H})$ be the power set of $\mathbb{H}$, let $\mathcal{P}_{0}(\mathbb{H})=\{\theta \in \mathcal{P}(\mathbb{H}): \theta$ is a finite set $\}$, let $\left(P_{I}\right)_{I \in \mathcal{P}_{0}(\mathbb{H})} \subseteq L\left(H_{\min \{0, \gamma\}}, H\right)$ satisfy for every $I \in$ $\mathcal{P}_{0}(\mathbb{H}), v \in H_{\min \{0, \gamma\}}$ that $P_{I}(v)=\sum_{h \in I}\left\langle(-A)^{-\min \{0, \gamma\}} h,(-A)^{\min \{0, \gamma\}} v\right\rangle_{H} h$, and let $O:[0, T] \times \Omega \rightarrow$ $H_{\gamma}$ be a stochastic process with continuous sample paths which satisfies for every $t \in[0, T]$ that 
$\left[O_{t}\right]_{\mathbb{P}, \mathcal{B}\left(H_{\gamma}\right)}=\int_{0}^{t} e^{(t-s) A} B d W_{s}$. Then there exist $\left(\mathbb{F}_{t}\right)_{t \in[0, T]}$-adapted stochastic processes $X^{I}:[0, T] \times$ $\Omega \rightarrow P_{I}(H), I \in \mathcal{P}_{0}(\mathbb{H})$, with continuous sample paths such that for every $I \in \mathcal{P}_{0}(\mathbb{H}), t \in[0, T]$ it holds that

$$
X_{t}^{I}=e^{t A} P_{I} \xi+\int_{0}^{t} e^{(t-s) A} P_{I} F\left(X_{s}^{I}\right) d s+P_{I} O_{t}
$$

Proof of Lemma 5.9. Throughout this proof let $\Phi: H_{1 / 2} \rightarrow[0, \infty)$ be the function which satisfies for every $w \in H_{1 / 2}$ that

$$
\Phi(w)=\frac{3\left|c_{1}\right|^{2}}{8\left|c_{0}\right|}\left[\sup _{u \in H_{1 / 2} \backslash\{0\}} \frac{\|u\|_{L^{\infty}(\lambda ; \mathbb{R})}}{\|u\|_{H_{1 / 2}}}+\sup _{u \in H_{1 / 2} \backslash\{0\}} \frac{\|u\|_{L^{4}(\lambda ; \mathbb{R})}^{2}}{\|u\|_{H_{1 / 2}}^{2}}\right]^{2}\left(1+\|w\|_{H_{1 / 2}}^{2}\right)^{2},
$$

let $\mathcal{A}_{I}: P_{I}(H) \rightarrow P_{I}(H), I \in \mathcal{P}_{0}(\mathbb{H})$, be the linear operators which satisfy for every $I \in \mathcal{P}_{0}(\mathbb{H})$, $v \in P_{I}(H)$ that $\mathcal{A}_{I} v=A v$, and for every $I \in \mathcal{P}_{0}(\mathbb{H})$ let $\left(\mathcal{H}_{I, s},\langle\cdot, \cdot\rangle_{\mathcal{H}_{I, s}},\|\cdot\|_{\mathcal{H}_{I, s}}\right), s \in \mathbb{R}$, be a family of interpolation spaces associated to $-\mathcal{A}_{I}$. Note that item (iii) of Lemma 4.13 proves that for every $I \in \mathcal{P}_{0}(\mathbb{H}), v, w \in H_{1 / 2}$ it holds that

$$
\left\|P_{I} F(v)-P_{I} F(w)\right\|_{H} \leq\|F(v)-F(w)\|_{H} \leq \frac{\left|c_{1}\right|}{\sqrt{3} c_{0}}\left(\|v\|_{H_{1 / 2}}+\|w\|_{H_{1 / 2}}\right)\|v-w\|_{H_{H_{1} / 2}}
$$

Moreover, observe that Corollary 4.24 (with $\iota=1 / 2, v=v, w=w$ for $v, w \in H_{1 / 2}$ in the notation of Corollary 4.24) shows that for every $I \in \mathcal{P}_{0}(\mathbb{H}), v, w \in P_{I}(H) \subseteq H_{1 / 2}$ it holds that

$$
\begin{aligned}
& \left\langle v, P_{I} F(v+w)\right\rangle_{H}=\left\langle P_{I} v, F(v+w)\right\rangle_{H}=\langle v, F(v+w)\rangle_{H} \\
& \leq \frac{3\left|c_{1}\right|^{2}}{8\left|c_{0}\right|}\left[\sup _{u \in H_{1 / 2} \backslash\{0\}} \frac{\|u\|_{L^{\infty}(\lambda ; \mathbb{R})}}{\|u\|_{H_{1 / 2}}}+\sup _{u \in H_{1 / 2} \backslash\{0\}} \frac{\|u\|_{L^{4}(\lambda ; \mathbb{R})}^{2}}{\|u\|_{H_{1 / 2}}^{2}}\right]^{2}\left(\|v\|_{H}^{2}+\|w\|_{H_{1 / 2}}^{2}\right)\|w\|_{H_{1 / 2}}^{2}+\|v\|_{H_{1 / 2}}^{2} \\
& \leq \Phi(w)\left(1+\|v\|_{H}^{2}\right)+\|v\|_{H_{1 / 2}}^{2}<\infty .
\end{aligned}
$$

Combining (259) and Corollary 2.4 (with $\left(H,\langle\cdot, \cdot\rangle_{H},\|\cdot\|_{H}\right)=\left(P_{I}(H),\langle\cdot, \cdot\rangle_{H},\|\cdot\|_{H}\right), \mathbb{H}=I, \mathfrak{v}_{e_{n}}=$ $-c_{0} \pi^{2} n^{2}, A=\mathcal{A}_{I},\left(H_{s}\right)_{s \in \mathbb{R}}=\left(\mathcal{H}_{I, s}\right)_{s \in \mathbb{R}}, T=T, s=0, C=\left|c_{1}\right| / c_{0}, c=1, \delta=1 / 2, \kappa=1 / 2$, $F=\left(P_{I}(H) \ni x \mapsto P_{I} F(x) \in P_{I}(H)\right), \Phi=\left(P_{I}(H) \ni x \mapsto \Phi(x) \in[0, \infty)\right),\left(\Omega, \mathcal{F}, \mathbb{P},\left(\mathbb{F}_{t}\right)_{t \in[0, T]}\right)=$ $\left(\Omega, \mathcal{F}, \mathbb{P},\left(\mathbb{F}_{t}\right)_{t \in[0, T]}\right), \xi=\left(\Omega \ni \omega \mapsto P_{I} \xi(\omega) \in P_{I}(H)\right), O=\left([0, T] \times \Omega \ni(t, \omega) \mapsto P_{I} O_{t}(\omega) \in\right.$ $\left.P_{I}(H)\right)$ for $I \in \mathcal{P}_{0}(\mathbb{H}), n \in\left\{m \in \mathbb{N}: e_{m} \in P_{I}(\mathbb{H})\right\}$ in the notation of Corollary 2.4) therefore completes the proof of Lemma 5.9.

Theorem 5.10. Let $\lambda: \mathcal{B}((0,1)) \rightarrow[0,1]$ be the Lebesgue-Borel measure on $(0,1)$, for every measure space $(\Omega, \mathcal{F}, \mu)$, every measurable space $(S, \mathcal{S})$, every set $R$, and every function $f: \Omega \rightarrow R$ let $[f]_{\mu, \mathcal{S}}=\{g: \Omega \rightarrow S:(\exists D \in \mathcal{F}:[\mu(D)=0$ and $\{\omega \in \Omega: f(\omega) \neq g(\omega)\} \subseteq D])$ and $(\forall D \in$ $\left.\left.\mathcal{S}: g^{-1}(D) \in \mathcal{F}\right)\right\}$, let $T, \varepsilon, c_{0} \in(0, \infty), c_{1} \in \mathbb{R}, \beta \in(-1 / 4, \infty), \gamma \in(1 / 4, \min \{1,1 / 2+\beta\})$, $\left(H,\langle\cdot, \cdot\rangle_{H},\|\cdot\|_{H}\right)=\left(L^{2}(\lambda ; \mathbb{R}),\langle\cdot, \cdot\rangle_{L^{2}(\lambda ; \mathbb{R})},\|\cdot\|_{L^{2}(\lambda ; \mathbb{R})}\right)$, let $\left(e_{n}\right)_{n \in \mathbb{N}} \subseteq H$ satisfy for every $n \in \mathbb{N}$ that $e_{n}=\left[(\sqrt{2} \sin (n \pi x))_{x \in(0,1)}\right]_{\lambda, \mathcal{B}(\mathbb{R})}$, let $A: D(A) \subseteq H \rightarrow H$ be the linear operator which satisfies $D(A)=\left\{v \in H: \sum_{n=1}^{\infty}\left|n^{2}\left\langle e_{n}, v\right\rangle_{H}\right|^{2}<\infty\right\}$ and $\forall v \in D(A): A v=-\sum_{n=1}^{\infty} c_{0} \pi^{2} n^{2}\left\langle e_{n}, v\right\rangle_{H} e_{n}$, let $\left(H_{r},\langle\cdot, \cdot\rangle_{H_{r}},\|\cdot\|_{H_{r}}\right), r \in \mathbb{R}$, be a family of interpolation spaces associated to $-A$ (cf., e.g., [37, Section 3.7]), for every $v \in W^{1,2}((0,1), \mathbb{R})$ let $\partial v \in H$ satisfy for every $\varphi \in \mathcal{C}_{c p t}^{\infty}((0,1), \mathbb{R})$ that $\left\langle\partial v,[\varphi]_{\lambda, \mathcal{B}(\mathbb{R})}\right\rangle_{H}=-\left\langle v,\left[\varphi^{\prime}\right]_{\lambda, \mathcal{B}(\mathbb{R})}\right\rangle_{H}$, let $(\Omega, \mathcal{F}, \mathbb{P})$ be a probability space with a normal filtration $\left(\mathbb{F}_{t}\right)_{t \in[0, T]}$, let $\left(W_{t}\right)_{t \in[0, T]}$ be an $\operatorname{Id}_{H}$-cylindrical $\left(\mathbb{F}_{t}\right)_{t \in[0, T]}$-Wiener process, let $B \in \operatorname{HS}\left(H, H_{\beta}\right)$, and let $\xi: \Omega \rightarrow H_{\gamma+\varepsilon}$ be an $\mathbb{F}_{0} / \mathcal{B}\left(H_{\gamma+\varepsilon}\right)$-measurable function. Then 
(i) there exists a unique continuous function $F: H_{1 / 8} \rightarrow H_{-1 / 2}$ which satisfies for every $v \in H_{1 / 2}$ that $F(v)=c_{1} v \partial v$ and

(ii) there exists an up to indistinguishability unique $\left(\mathbb{F}_{t}\right)_{t \in[0, T] \text {-adapted stochastic process }}$ $X:[0, T] \times \Omega \rightarrow H_{\gamma}$ with continuous sample paths which satisfies for every $t \in[0, T]$ that

$$
\left[X_{t}\right]_{\mathbb{P}, \mathcal{B}\left(H_{\gamma}\right)}=\left[e^{t A} \xi+\int_{0}^{t} e^{(t-s) A} F\left(X_{s}\right) d s\right]_{\mathbb{P}, \mathcal{B}\left(H_{\gamma}\right)}+\int_{0}^{t} e^{(t-s) A} B d W_{s} .
$$

Proof of Theorem 5.10. Throughout this proof let $f: H_{1 / 2} \rightarrow H$ be the function which satisfies for every $v \in H_{1 / 2}$ that $f(v)=c_{1} v \partial v$, let $\mathbb{H}=\left\{e_{n}: n \in \mathbb{N}\right\}$, let $\mathcal{P}(\mathbb{H})$ be the power set of $\mathbb{H}$, let $\mathcal{P}_{0}(\mathbb{H})=\{\theta \in \mathcal{P}(\mathbb{H}): \theta$ is a finite set $\}$, let $\left(P_{I}\right)_{I \in \mathcal{P}(\mathbb{H})} \subseteq L(H)$ satisfy for every $I \in \mathcal{P}(\mathbb{H}), v \in H$ that $P_{I}(v)=\sum_{h \in I}\langle h, v\rangle_{H} h$, let $\nu=(2-4 \min \{\gamma, 1 / 2\}) / 3, \eta \in(0, \min \{2 \varepsilon, 1+2(\beta-\gamma), 2(1-\gamma-\nu)\})$, and let $\left(I_{n}\right)_{n \in \mathbb{N}} \subseteq \mathcal{P}_{0}(\mathbb{H})$ satisfy for every $n \in \mathbb{N}$ that $I_{n}=\left\{e_{1}, \ldots, e_{n}\right\}$. Note that item (ii) of Corollary 4.18 (with $F=f, \bar{F}=F$ in the notation of Corollary 4.18) establishes item (ii). Next we intend to apply Blömker \& Jentzen [4, Theorem 3.1] to prove item (iii). For this observe that Lemma 5.5 (with $H=H, \mathbb{H}=\mathbb{H}, \mathfrak{v}_{e_{n}}=-c_{0} \pi^{2} n^{2}, A=A, H_{r}=H_{r}, T=T, \beta=\beta, \gamma=\gamma$, $B=B,(\Omega, \mathcal{F}, \mathbb{P})=(\Omega, \mathcal{F}, \mathbb{P}),\left(W_{t}\right)_{t \in[0, T]}=\left(W_{t}\right)_{t \in[0, T]}$ for $n \in \mathbb{N}, r \in \mathbb{R}$ in the notation of Lemma [5.5) ensures that there exists an $\left(\mathbb{F}_{t}\right)_{t \in[0, T]}$-adapted stochastic process $O:[0, T] \times \Omega \rightarrow H_{\gamma}$ with continuous sample paths which satisfies for every $t \in[0, T]$ that

$$
\left[O_{t}\right]_{\mathbb{P}, \mathcal{B}\left(H_{\gamma}\right)}=\int_{0}^{t} e^{(t-s) A} B d W_{s} .
$$

Note that (262) and Lemma 5.9 (with $c_{0}=c_{0}, c_{1}=c_{1}, H=H, \mathbb{H}=\mathbb{H}, \mathfrak{v}_{e_{n}}=-c_{0} \pi^{2} n^{2}$, $e_{n}=e_{n}, A=A, H_{r}=H_{r}, F=f, T=T, \beta=\beta, \gamma=\gamma, B=B,(\Omega, \mathcal{F}, \mathbb{P})=(\Omega, \mathcal{F}, \mathbb{P})$, $\left(\mathbb{F}_{t}\right)_{t \in[0, T]}=\left(\mathbb{F}_{t}\right)_{t \in[0, T]},\left(W_{t}\right)_{t \in[0, T]}=\left(W_{t}\right)_{t \in[0, T]}, \xi=(\Omega \ni \omega \mapsto \xi(\omega) \in H), P_{I}=P_{I}, O=O$ for $n \in \mathbb{N}, r \in \mathbb{R}, I \in \mathcal{P}_{0}(\mathbb{H})$ in the notation of Lemma $[5.9)$ show that there exist $\left(\mathbb{F}_{t}\right)_{t \in[0, T] \text {-adapted }}$ stochastic processes $X^{I}:[0, T] \times \Omega \rightarrow P_{I}(H), I \in \mathcal{P}_{0}(\mathbb{H})$, with continuous sample paths which satisfy for every $I \in \mathcal{P}_{0}(\mathbb{H}), t \in[0, T]$ that

$$
X_{t}^{I}=e^{t A} P_{I} \xi+\int_{0}^{t} e^{(t-s) A} P_{I} f\left(X_{s}^{I}\right) d s+P_{I} O_{t}
$$

Next let $\Sigma \in \mathcal{F}$ be the set which satisfies that

$$
\Sigma=\left\{\omega \in \Omega: \sup _{n \in \mathbb{N}}\left(n^{\eta} \sup _{t \in[0, T]}\left\|O_{t}(\omega)-P_{I_{n}} O_{t}(\omega)\right\|_{H_{\gamma}}\right)<\infty\right\},
$$

let $\mathcal{O}:[0, T] \times \Omega \rightarrow H_{\gamma}$ be the stochastic process which satisfies for every $t \in[0, T], \omega \in \Omega$ that

$$
\mathcal{O}_{t}(\omega)= \begin{cases}O_{t}(\omega) & : \omega \in \Sigma \\ -e^{t A} \xi(\omega)-\int_{0}^{t} e^{(t-s) A} f(0) d s & : \omega \in(\Omega \backslash \Sigma),\end{cases}
$$

and let $\mathcal{X}^{I}:[0, T] \times \Omega \rightarrow P_{I}(H), I \in \mathcal{P}_{0}(\mathbb{H})$, be the stochastic processes which satisfy for every $I \in \mathcal{P}_{0}(\mathbb{H}), t \in[0, T], \omega \in \Sigma$ that

$$
\mathcal{X}_{t}^{I}(\omega)= \begin{cases}X_{t}^{I}(\omega) & : \omega \in \Sigma \\ 0 & : \omega \in(\Omega \backslash \Sigma)\end{cases}
$$


Moreover, note that the fact that $(\gamma+\nu) \in(0,1)$ shows that for every $t \in(0, T]$ it holds that

$$
\left\|e^{t A}\right\|_{L\left(H_{-\nu}, H_{\gamma}\right)} \leq t^{-\gamma-\nu} \leq t^{-\gamma-\nu-(\eta / 2)} T^{\eta / 2}
$$

This ensures that

$$
\sup _{t \in(0, T]}\left(t^{\gamma+\nu+(\eta / 2)}\left\|e^{t A}\right\|_{L\left(H_{-\nu}, H_{\gamma}\right)}\right)<\infty .
$$

In addition, observe that the fact that $(\gamma+\nu+(\eta / 2)) \in(0,1)$ implies that for every $n \in \mathbb{N}, t \in[0, T]$ it holds that

$$
\begin{aligned}
& \left\|P_{\mathbb{H} \backslash I_{n}} e^{t A}\right\|_{L\left(H_{-\nu}, H_{\gamma}\right)}=\left\|(-A)^{-\eta / 2} P_{\mathbb{H} \backslash I_{n}}(-A)^{\gamma+\nu+(\eta / 2)} e^{t A}\right\|_{L(H)} \\
& \leq\left\|(-A)^{-\eta / 2} P_{\mathbb{H} \backslash I_{n}}\right\|_{L(H)}\left\|(-A)^{\gamma+\nu+(\eta / 2)} e^{t A}\right\|_{L(H)} \leq\left[c_{0} \pi^{2}(n+1)^{2}\right]^{-\eta / 2} t^{-\gamma-\nu-(\eta / 2)} .
\end{aligned}
$$

This proves that

$$
\sup _{n \in \mathbb{N}} \sup _{t \in(0, T]}\left(t^{\gamma+\nu+(\eta / 2)} n^{\eta}\left\|e^{t A}-P_{I_{n}} e^{t A}\right\|_{L\left(H_{-\nu}, H_{\gamma}\right)}\right)<\infty .
$$

Next note that the fact that for every $x \in(1 / 8,1 / 2]$ it holds that

$$
\left(\frac{(2-4 x)}{3}\right) \in\left(\left[\frac{1}{2}-x, \frac{1}{2}\right] \cap\left(\frac{3}{4}-2 x, \infty\right)\right)
$$

and Lemma4.16 (with $\gamma=\min \{\gamma, 1 / 2\}, \nu=(2-4 \min \{\gamma, 1 / 2\}) / 3$ in the notation of Lemma 4.16) ensure that there exists $C \in[0, \infty)$ such that for every $v, w \in H_{\gamma} \subseteq H_{\min \{\gamma, 1 / 2\}}$ it holds that

$$
\begin{aligned}
& \|F(v)-F(w)\|_{H_{-}(2-4 \min \{\gamma, 1 / 2\}) / 3} \leq C\|v-w\|_{H_{\min \{\gamma, 1 / 2\}}}\left(1+\|v\|_{H_{\min \{\gamma, 1 / 2\}}}+\|w\|_{H_{\min \{\gamma, 1 / 2\}}}\right) \\
& \leq C\left[\max \left\{1,\left\|(-A)^{\min \{0,(1 / 2)-\gamma\}}\right\|_{L(H)}\right\}\right]^{2}\|v-w\|_{H_{\gamma}}\left(1+\|v\|_{H_{\gamma}}+\|w\|_{H_{\gamma}}\right) .
\end{aligned}
$$

This demonstrates that there exists $C \in \mathbb{R}$ such that for every $v, w \in H_{\gamma}$ it holds that

$$
\|F(v)-F(w)\|_{H_{-\nu}}=\|F(v)-F(w)\|_{H_{-(2-4 \min \{\gamma, 1 / 2\}) / 3}} \leq C\|v-w\|_{H_{\gamma}}\left(1+\|v\|_{H_{\gamma}}+\|w\|_{H_{\gamma}}\right) .
$$

Furthermore, observe that (264), the fact that $\eta \in(0,1+2(\beta-\gamma)$ ), and Lemma 5.8 (with $T=T$, $\beta=\beta, \gamma=\gamma, B=B, P_{I}=P_{I},(\Omega, \mathcal{F}, \mathbb{P})=(\Omega, \mathcal{F}, \mathbb{P}),\left(W_{t}\right)_{t \in[0, T]}=\left(W_{t}\right)_{t \in[0, T]}, O=O$ for $I \in \mathcal{P}(\mathbb{H})$ in the notation of Lemma [5.8) show that $\mathbb{P}(\Sigma)=1$. This and (265) prove that

$$
\mathbb{P}\left(\forall t \in[0, T]: \mathcal{O}_{t}=O_{t}\right)=1 .
$$

In the next step we note that the fact that $f(0)=0$ ensures that for every $n \in \mathbb{N}, \omega \in(\Omega \backslash \Sigma)$, $t \in[0, T]$ it holds that

$$
\left\|P_{\mathbb{H} \backslash I_{n}}\left(\mathcal{O}_{t}(\omega)+e^{t A} \xi(\omega)\right)\right\|_{H_{\gamma}}=\left\|P_{\mathbb{H} \backslash I_{n}} \int_{0}^{t} e^{(t-s) A} f(0) d s\right\|_{H_{\gamma}}=0 .
$$

Furthermore, observe that for every $n \in \mathbb{N}, \omega \in \Omega, t \in[0, T]$ it holds that

$$
\left\|P_{\mathbb{H} \backslash I_{n}} e^{t A} \xi(\omega)\right\|_{H_{\gamma}} \leq\left(\sup _{h \in \mathbb{H} \backslash I_{n}}\left|\mathfrak{v}_{h}\right|^{-\eta / 2}\right)\|\xi(\omega)\|_{H_{\gamma+(\eta / 2)}}=\left[c_{0} \pi^{2}(n+1)^{2}\right]^{-\eta / 2}\|\xi(\omega)\|_{H_{\gamma+(\eta / 2)}} .
$$

Combining this, (264), (275), and the triangle inequality demonstrates that for every $\omega \in \Omega$ it holds that

$$
\sup _{n \in \mathbb{N}}\left(n^{\eta} \sup _{t \in[0, T]}\left\|P_{\mathbb{H} \backslash I_{n}}\left(\mathcal{O}_{t}(\omega)+e^{t A} \xi(\omega)\right)\right\|_{H_{\gamma}}\right)<\infty
$$


Moreover, note that (263) holds that

$$
\mathcal{X}_{t}^{I}(\omega)=e^{t A} P_{I} \xi(\omega)+\int_{0}^{t} e^{(t-s) A} P_{I} f\left(\mathcal{X}_{s}^{I}(\omega)\right) d s+P_{I} \mathcal{O}_{t}(\omega)
$$

In addition, observe that the fact that $\mathcal{O}:[0, T] \times \Omega \rightarrow H_{\gamma}$ has continuous sample paths establishes for every $\omega \in \Omega$ that

$$
\sup _{t \in[0, T]}\left\|\mathcal{O}_{t}(\omega)\right\|_{H_{\gamma}}<\infty
$$

The fact that $\gamma<1$, (278), and Lemma 5.3 (with $F=f, T=T, \iota=\gamma, \gamma=\gamma, \xi=\xi(\omega)$, $O_{t}^{I}=P_{I} \mathcal{O}_{t}(\omega), X_{t}^{I}=\mathcal{X}_{t}^{I}(\omega)$ for $n \in \mathbb{N}, I \in \mathcal{P}_{0}(\mathbb{H}), t \in[0, T], \omega \in \Omega$ in the notation of Lemma 5.3) therefore prove that for every $\omega \in \Omega$ it holds that

$$
\sup _{n \in \mathbb{N}} \sup _{t \in[0, T]}\left\|\mathcal{X}_{t}^{I_{n}}(\omega)\right\|_{H_{\gamma}}<\infty
$$

Furthermore, note that item (ii) and (278) show that for every $I \in \mathcal{P}_{0}(\mathbb{H}), \omega \in \Omega, t \in[0, T]$ it holds that

$$
\mathcal{X}_{t}^{I}(\omega)=e^{t A} P_{I} \xi(\omega)+\int_{0}^{t} e^{(t-s) A} P_{I} F\left(\mathcal{X}_{s}^{I}(\omega)\right) d s+P_{I} \mathcal{O}_{t}(\omega)
$$

Combining the fact that $0<\gamma+\nu+(\eta / 2)<1$, (268), (270), (273), (277), and (280) with Blömker \& Jentzen [4, Theorem 3.1] (with $T=T,(\Omega, \mathcal{F}, \mathbb{P})=(\Omega, \mathcal{F}, \mathbb{P}), V=H_{\gamma}, W=H_{-\nu}, P_{n}=P_{I_{n}}$, $\alpha=\gamma+\nu+(\eta / 2), \gamma=\eta, S=\left((0, T] \ni s \mapsto e^{s A} \in L\left(H_{-\nu}, H_{\gamma}\right)\right), F=\left(H_{\gamma} \ni v \mapsto F(v) \in H_{-\nu}\right)$, $O_{t}=\mathcal{O}_{t}+e^{t A} \xi, X_{t}^{n}=\mathcal{X}_{t}^{I_{n}}$ for $t \in[0, T], n \in \mathbb{N}$ in the notation of Blömker \& Jentzen [4, Theorem 3.1]) therefore shows that

(a) there exists a unique stochastic process $X:[0, T] \times \Omega \rightarrow H_{\gamma}$ with continuous sample paths which satisfies for every $t \in[0, T]$ that

$$
X_{t}=e^{t A} \xi+\int_{0}^{t} e^{(t-s) A} F\left(X_{s}\right) d s+\mathcal{O}_{t}
$$

and

(b) there exists a $\mathcal{F} / \mathcal{B}([0, \infty))$-measurable function $K: \Omega \rightarrow[0, \infty)$ such that for every $\omega \in \Omega$, $n \in \mathbb{N}$ it holds that

$$
\sup _{t \in[0, T]}\left\|X_{t}(\omega)-\mathcal{X}_{t}^{I_{n}}(\omega)\right\|_{H_{\gamma}} \leq \frac{K(\omega)}{n^{\eta}} .
$$

Observe that the fact that for every $n \in \mathbb{N}$ it holds that $\left(\mathcal{X}_{t}^{I_{n}}\right)_{t \in[0, T]}$ is $\left(\mathbb{F}_{t}\right)_{t \in[0, T]}$-adapted and item (b) imply that $\left(X_{t}\right)_{t \in[0, T]}$ is $\left(\mathbb{F}_{t}\right)_{t \in[0, T]}$-adapted. Combining (262), (274), and item (国) hence establishes item (iii). This completes the proof of Theorem 5.10 .

\section{Acknowledgements}

This project has been partially supported through the SNSF-Research project 200021_156603 "Numerical approximations of nonlinear stochastic ordinary and partial differential equations". 


\section{References}

[1] Aliprantis, C. D., And Border, K. C. Infinite dimensional analysis, third ed. Springer, Berlin, 2006. A hitchhiker's guide.

[2] Behzadan, A., And Holst, M. Multiplication in Sobolev spaces, revisited. arXiv:1512.07379 (2017), 24 pages.

[3] Bertini, L., Cancrini, N., and Jona-Lasinio, G. The stochastic Burgers equation. Comm. Math. Phys. 165, 2 (1994), 211-232.

[4] Blömker, D., And Jentzen, A. Galerkin approximations for the stochastic Burgers equation. SIAM J. Numer. Anal. 51, 1 (2013), 694-715.

[5] Brezis, H. Functional analysis, Sobolev spaces and partial differential equations. Universitext. Springer, New York, 2011.

[6] Brzeźniak, Z., Debbi, L., And Goldys, B. Ergodic properties of fractional stochastic Burgers equation. Glob. Stoch. Anal. 1, 2 (2011), 149-174.

[7] Da Prato, G., Debussche, A., and Temam, R. Stochastic Burgers' equation. NoDEA Nonlinear Differential Equations Appl. 1, 4 (1994), 389-402.

[8] Da Prato, G., And Gatarek, D. Stochastic Burgers equation with correlated noise. Stochastics Stochastics Rep. 52, 1-2 (1995), 29-41.

[9] Da Prato, G., And ZabczyK, J. Stochastic equations in infinite dimensions, vol. 44 of Encyclopedia of Mathematics and its Applications. Cambridge University Press, Cambridge, 1992.

[10] Da Prato, G., And Zabczyk, J. Ergodicity for infinite-dimensional systems, vol. 229 of London Mathematical Society Lecture Note Series. Cambridge University Press, Cambridge, 1996.

[11] Da Prato, G., And Zabczyk, J. Stochastic equations in infinite dimensions, second ed., vol. 152 of Encyclopedia of Mathematics and its Applications. Cambridge University Press, Cambridge, 2014.

[12] Dong, Z., And Xu, T. G. One-dimensional stochastic Burgers equation driven by Lévy processes. J. Funct. Anal. 243, 2 (2007), 631-678.

[13] Fujiwara, D. Concrete characterization of the domains of fractional powers of some elliptic differential operators of the second order. Proc. Japan Acad. 43 (1967), 82-86.

[14] Grecksch, W., And Tudor, C. Stochastic Evolution Equations: A Hilbert Space Approach. Wiley, New York, 1996.

[15] Gyöngy, I. Existence and uniqueness results for semilinear stochastic partial differential equations. Stochastic Process. Appl. 73, 2 (1998), 271-299.

[16] Gyöngy, I., And Nualart, D. On the stochastic Burgers' equation in the real line. Ann. Probab. 27, 2 (1999), 782-802. 
[17] Hausenblas, E., And GiRi, A. K. Stochastic Burgers equation with polynomial nonlinearity driven by Lévy process. Commun. Stoch. Anal. 7, 1 (2013), 91-112.

[18] Jentzen, A., And Kloeden, P. Taylor Approximations for Stochastic Partial Differential Equations, vol. 83 of CBMS-NSF Regional Conference Series in Applied Mathematics. Society for Industrial and Applied Mathematics (SIAM), Philadelphia, PA, 2012.

[19] Jentzen, A., Lindner, F., And Pušnik, P. On the Alekseev-Gröbner formula in Banach spaces. arXiv:1810.10030 (2018), 36 pages.

[20] Jentzen, A., And Pušnik, P. Exponential moments for numerical approximations of stochastic partial differential equations. Stoch. Partial Differ. Equ. Anal. Comput. 6, 4 (2018), 565-617.

[21] Jentzen, A., AND PUŠnik, P. Strong convergence rates for an explicit numerical approximation method for stochastic evolution equations with non-globally Lipschitz continuous nonlinearities. IMA Journal of Numerical Analysis (April 2019). https://doi.org/10.1093/imanum/drz009, in press, published online: 12 April 2019 (Accessed 1 August 2019).

[22] Jentzen, A., And RöCKner, M. Regularity analysis of stochastic partial differential equations with nonlinear multiplicative trace class noise. J. Differential Equations 252, 1 (2012), 114-136.

[23] Jentzen, A., Salimova, D., And Welti, T. Strong convergence for explicit space-time discrete numerical approximation methods for stochastic Burgers equations. J. Math. Anal. Appl. 469, 2 (2019), 661-704.

[24] Kallenberg, O. Foundations of modern probability. Probability and its Applications (New York). Springer-Verlag, New York, 1997.

[25] KIm, J. U. On the stochastic Burgers equation with a polynomial nonlinearity in the real line. Discrete Contin. Dyn. Syst. Ser. B 6, 4 (2006), 835-866.

[26] Klenke, A. Probability theory. Universitext. Springer-Verlag London Ltd., London, 2008. A comprehensive course, Translated from the 2006 German original.

[27] Kloeden, P. E., And Neuenkirch, A. The pathwise convergence of approximation schemes for stochastic differential equations. LMS J. Comput. Math. 10 (2007), 235-253.

[28] Lewis, P., And Nualart, D. Stochastic Burgers' equation on the real line: regularity and moment estimates. Stochastics 90, 7 (2018), 1053-1086.

[29] Liu, W., AND RöCKner, M. Stochastic partial differential equations: an introduction. Universitext. Springer, Cham, 2015.

[30] Lunardi, A. Interpolation theory, vol. 16 of Appunti. Scuola Normale Superiore di Pisa (Nuova Serie) [Lecture Notes. Scuola Normale Superiore di Pisa (New Series)]. Edizioni della Normale, Pisa, 2018. Third edition [of MR2523200]. 
[31] Mazzonetto, S., And Salimova, D. Existence, uniqueness, and numerical approximations for stochastic Burgers equations. arXiv:1901.09288 (2019), 23 pages.

[32] Nirenberg, L. On elliptic partial differential equations. Ann. Scuola Norm. Sup. Pisa (3) 13 (1959), 115-162.

[33] Prévôt, C., And Röckner, M. A concise course on stochastic partial differential equations, vol. 1905 of Lecture Notes in Mathematics. Springer, Berlin, 2007.

[34] Röckner, M., Zhu, R., And ZHu, X. Local existence and non-explosion of solutions for stochastic fractional partial differential equations driven by multiplicative noise. Stochastic Process. Appl. 124, 5 (2014), 1974-2002.

[35] Rozovskil, B. L. Stochastic evolution systems, vol. 35 of Mathematics and its Applications (Soviet Series). Kluwer Academic Publishers Group, Dordrecht, 1990. Linear theory and applications to nonlinear filtering, Translated from the Russian by A. Yarkho.

[36] SearcóId, M. O. Metric spaces. Springer Undergraduate Mathematics Series. SpringerVerlag London, Ltd., London, 2007.

[37] Sell, G. R., And You, Y. Dynamics of evolutionary equations, vol. 143 of Applied Mathematical Sciences. Springer-Verlag, New York, 2002.

[38] Triebel, H. Interpolation theory, function spaces, differential operators, vol. 18 of NorthHolland Mathematical Library. North-Holland Publishing Co., Amsterdam-New York, 1978.

[39] Walsh, J. B. An introduction to stochastic partial differential equations, vol. 1180 of Lecture Notes in Mathematics. Springer, Berlin, 1986.

[40] Zhang, X. Regularities for semilinear stochastic partial differential equations. J. Funct. Anal. 249, 2 (2007), 454-476. 